\title{
Effects of Fluctuations on Propagating Fronts
}

\author{
Debabrata Panja \\ Institute for Theoretical Physics, Universiteit van Amsterdam, Valckenierstraat \\ 65, 1018 XE Amsterdam, The Netherlands
}

\begin{abstract}
Propagating fronts are seen in varieties of non-equilibrium pattern forming systems in Physics, Chemistry and Biology. In the last two decades, many researchers have contributed to the understanding of the underlying dynamics of the propagating fronts. Of these, the deterministic and mean-field dynamics of the fronts were mostly understood in late 1980s and 1990s. On the other hand, although the earliest work on the effect of fluctuations on propagating fronts dates back to early 1980s, the subject of fluctuating fronts did not reach its adolescence until the mid 1990s. From there onwards the last few years witnessed a surge in activities in the effect of fluctuations on propagating fronts. Scores of papers have been written on this subject since then, contributing to a significant maturity of our understanding, and only recently a full picture of fluctuating fronts has started to emerge. This review is an attempt to collect all the works on fluctuating (propagating) fronts in a coherent and cogent manner in proper perspective. It is based on the idea of making our knowledge in this field available to a broader audience, and it is also expected to help to collect bits and pieces of loose thread-ends together for possible further investigation.
\end{abstract}

\section{Contents}

1 Front Propagation in Far from Equilibrium Systems 3

1.1 Propagating Fronts into Unstable States: Deterministic Systems 3

1.2 Fluctuating (and propagating) Fronts: A Separate Paradigm 10

$\begin{array}{lll}1.3 & \text { A Note on the Notations } & 15\end{array}$

$2 \quad$ Fluctuating Fronts in Discrete Particle and Lattice Systems $\quad 16$

$\begin{array}{lll}2.1 & \text { Summary of Known Results } & 16\end{array}$

2.2 Derivation of $1 / \ln ^{2} N$ Convergence of the Asymptotic Front Speed to $v^{*}$ for Fluctuating "Pulled" Fronts as $N \rightarrow \infty$ 
2.3 The Case of Intermediately Large Values of $N$, Foremost Occupied Lattice Site, Tip Region of the Front and All That

2.4 The weakly pushed nature of fluctuating "pulled" fronts

2.5 The Large $N$ Asymptotic Scaling of Front Diffusion Coefficient $D_{f}$ for Fluctuating "Pulled" Fronts

2.6 The Case of Small Values of $N$

2.7 Convergence of the Asymptotic Front Speed to $v^{\dagger}$ for Fluctuating Pushed Fronts as $N \rightarrow \infty$

2.8 Epilogue I

$3 \quad$ Field-theory of Fluctuating Fronts: External Fluctuations

3.1 External Fluctuations, Multiplicative Noise and Novikov's Theorem

3.2 Application of Novikov's Theorem to Reaction-Diffusion Systems: Effects of Multiplicative Noise on Specific Models

3.3 Fluctuating Pulled Fronts with Multiplicative Noise in ReactionDiffusion Systems: Diffusive vs. Subdiffusive Wandering of the Goldstone Mode

3.4 Fluctuating Fronts with Multiplicative Noise in Reaction-Diffusion Systems and Kinetic Roughening

4 Field-theory of Fluctuating Fronts: Internal Fluctuations

4.1 Fluctuating "Pulled" Fronts in Stochastic Fisher-KolmogorovPetrovsky-Piscunov (sFKPP) Equation

$\begin{array}{llr}6 & \text { Acknowledgement } & 106\end{array}$

$\begin{array}{lr}\text { References } & 106\end{array}$ 


\section{Front Propagation in Far from Equilibrium Systems}

\subsection{Propagating Fronts into Unstable States: Deterministic Systems}

In pattern forming systems quite often situations occur where patches of different bulk phases get separated by fronts or interfaces. In such cases, the relevant dynamics of the system is usually dominated by the dynamics of these fronts. When the interface separates two thermodynamically stable phases, as in crystal-melt interfacial growth problems, the width of the interfacial zone is usually of atomic dimensions. For such systems, one often has to resort to a moving boundary description, in which the boundary conditions at the interface are determined phenomenologically or by microscopic considerations. A question that naturally arises for such interfaces is the influence of stochastic fluctuations on the motion and scaling properties of such interfaces.

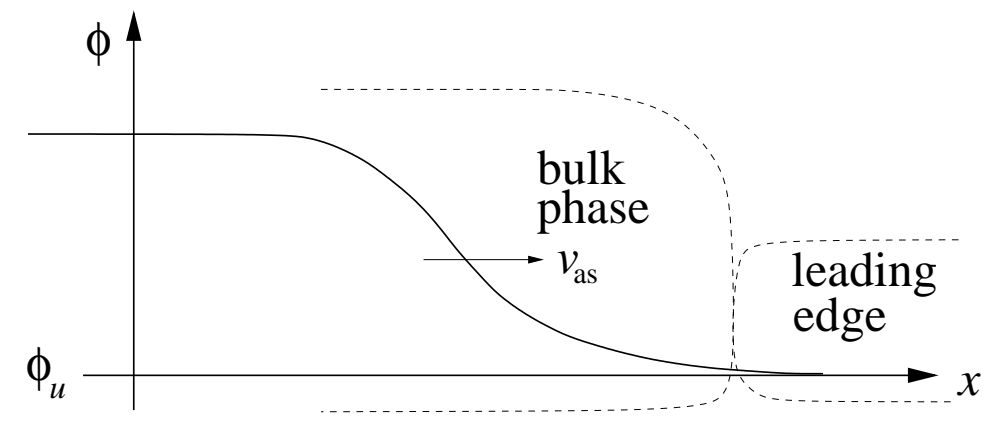

Fig. 1. A typical deterministic front propagating into a linearly unstable state $\phi_{u}$ and moving with speed $v_{\mathrm{as}}$ : at the leading edge, the deviation of the front field $\phi(x, t)$ from its unstable state value is infinitesimal and the nonlinear terms in the deterministic equation that describes the front can be neglected. For pulled fronts, the perturbation around the unstable state at the leading edge ahead of the front grows linearly and spreads with speed $v^{*}$, thereby pulling the rest of the front along with it at $v_{\text {as }}=v^{*}$ (hence the nomenclature). For pushed fronts, it is the bulk phase that drives the front propagation mechanism, as if the bulk phase (whose description requires nonlinear terms in the equation describing the front) pushes the leading edge from behind to achieve a front speed $>v^{*}$.

At the other extreme is a class of fronts that arise in pattern forming systems, where the occurrence of fronts or transition zones is fundamentally related to their inherent nonequilibrium nature, as they do not connect two thermodynamic equilibrium phases that are separated by a first order phase transition. In such cases - for example, chemical fronts [78], the temperature and density transition zones in thermal plumes [121], the domain walls separating domains of different orientations in rotating Rayleigh-Bénard convection [117], streamer fronts in discharges [39], or the aggregate fronts in diffusion limited aggregation [15] — the fronts are relatively wide and are therefore described by the same continuum equations that describe nonequilibrium bulk patterns. 
The lore in nonequilibrium pattern formation is that when the relevant length scales are large, (thermal) fluctuation effects are relatively small [26]. For this reason, in the first approximation, the dynamics of many pattern forming systems can be understood in terms of the deterministic dynamics of the basic patterns and coherent structures. The first questions to study for them are properties like the existence and the asymptotic speed of propagation of the front solutions in deterministic equations. For example, in one dimension, the existence of an asymptotic propagation speed $v_{\text {as }}$ for deterministic fronts means that in the comoving co-ordinate $x-v_{\text {as }} t$, moving w.r.t. to the laboratory co-ordinate $x$ at a speed $v_{\text {as }}$, the front profile approaches a fixed shape as $t \rightarrow \infty$. Given the dynamical equations, the interest is in the magnitude of $v_{\text {as }}$, and as well as in how the asymptotic front shape and speed are approached in time from a given initial configuration. In most cases of deterministic fronts, these equations are partial differential equations.

Instances of such fronts, where fluctuation effects are small (and therefore they can be neglected in favour of a deterministic description) are abundant in physics $[1,25,30,42,48,51,63,84,103,104,113,114]$, chemistry $[49,50,75,120]$ and biology $[17,41,58,82] .{ }^{1}$ As a result of detailed studies carried out in the last decade, it has emerged that in these systems, the dynamics is described by propagation of (deterministic) fronts into unstable states, i.e., the state of the system in the region far ahead of the front is linearly unstable [102]. These studies have classified deterministic fronts propagating into unstable states in two categories in a broad sense: the so-called pulled and pushed fronts (see Fig. 1). Pulled fronts are the fronts that propagate into a linearly unstable state, and for which the asymptotic front speed $v_{\text {as }}$ is the linear spreading speed $v^{*}$ of infinitesimal perturbations around the unstable state $[12,29,38,100]$. The name pulled fronts refer to the intuitive picture that at the leading edge of these fronts, ${ }^{2}$ the perturbation around the unstable state ahead of the front grows and spreads with speed $v^{*}$, while the rest of the front gets "pulled along" by the leading edge. On the other hand, fronts that propagate into a linearly unstable state and whose asymptotic speed is $>v^{*}$ are referred to as pushed fronts, as it is the nonlinear growth in the region behind the leading edge that pushes their front speeds to higher values. ${ }^{3}$ If the state is not linearly

1 On account of the fact that this article is about fluctuating fronts, these citations are representative and by no means complete. For a more comprehensive set of references, see Ref. [102], a review article on deterministic fronts.

2 The term leading edge, which will appear in this review article over and over again, is (and will be) used to denote the front region where the value of the front field $\phi$ is very close to its value at the the state it propagates into. In other words, in this region, the front evolution equation can be linearized around the value of $\phi$ at the the state it propagates into.

3 By definition, therefore, the asymptotic speed of a pushed front can be obtained only by solving the full nonlinear equation; in general it is not possible to do so except for special sets of parameter values. 
unstable, then $v^{*}$ is trivially zero, and in addition, there can also be fronts propagating into unstable states with $v^{*}=0$; in such cases front propagation is always dominated by the nonlinear growth in the front region itself, and hence fronts in these cases are in a sense pushed too. ${ }^{4}$

For a front propagating into linearly unstable state, the linear spreading speed $v^{*}$ is obtained from the time evolution of a localized initial perturbation around the unstable state. A beautiful analysis of this can be found in Sec. 2.1 of Ref. [102]. The knowledge of the existence or the magnitude of $v^{*}$ alone however does not answer the important questions like how and under what conditions one can expect a pulled front in the system, or when to expect a pushed front in the a given model. Addressing these questions satisfactorily is an involved process, and as already mentioned in footnote 4 , from the point of view of this review article, in Sec. 1.1 we present the basic necessary results by considering an example system. The equation that we choose for the illustration of the properties of pulled fronts is the so-called Fisher-Kolmogorov-PetrovskyPiscounov equation (we will refer to it as Fisher-Kolmogorov equation hereafter), which was at first used to model the spreading of advantageous genes in a population $[43,59]$. In this model, the density of the advantageous genes is denoted by $\phi(x, t)$, a non-negative quantity, whose dynamics is described by the equation

$$
\frac{\partial \phi}{\partial t}=\frac{\partial^{2} \phi}{\partial x^{2}}+\phi-\phi^{n} . \quad n>1, \quad \text { for example } 2 \text { or } 3
$$

Equation (1) has two stationary states, of which $\phi_{u}=0$ is (linearly) unstable and $\phi_{s}=1$ is stable. Therefore, if the system is prepared in a way such that these two states coexist in a certain region of space, then the stable state invades the unstable one and propagates into it. The profile of the resulting

4 At this point, I must warn the reader that I am being much more than just a little bit naive and simplistic in describing the broad classification of fronts propagating into unstable states in this manner. The actual issue of how $v^{*}$ comes out naturally for fronts propagating into unstable states and there onwards when one can expect a pulled front (possibly a further subclassification of coherent or incoherent pulled fronts) or a pushed front is a fairly involved subject in itself. For a proper analysis of it, the reader is encouraged to go through Sec. 2 of Ref. [102]. The point, once again, is that this is a review article on fluctuating (and propagating) fronts. From this point of view, it is enough to understand the basic issues that involve the classification in terms of pulled or pushed fronts in a broad sense. Such an attitude will be reflected all along Sec. 1.1, where I will highlight these basic features of pulled and pushed fronts in terms of examples and pictorial representations. Furthermore, in Sec. 1.1, I would completely leave out any discussion on deterministic pattern forming fronts such as Complex Ginzburg-Landau equation or Swift-Hohenberg equation etc., simply because literature on the effect of fluctuations in such pattern forming fronts do not exist in the literature. 
front is similar to that of Fig. 1.

To obtain the front solution admitted by Eq. (1), we rewrite it in a frame that moves w.r.t. the laboratory frame at a constant speed $v$, by means of a change of variables from $x$ to the comoving co-ordinate $\xi \equiv x-v t$ as

$$
\frac{\partial \phi}{\partial t}-v \frac{\partial \phi}{\partial \xi}=\frac{\partial^{2} \phi}{\partial \xi^{2}}+\phi-\phi^{n}
$$

and look for a stationary solution of $\phi$ in this comoving frame. The crucial relevance of the growth and spreading of infinitesimal perturbations enters naturally in this front solution, as the propagating infinitesimal perturbations around the unstable state in the leading edge ahead of the front sets on the instability making way for further growth. At the leading edge of the front, the $\phi$-values are very close to the unstable state value, i.e., $\phi \ll 1$, and one can neglect the nonlinear term $\phi^{n}$ compared to $\phi$ in Eq. (2). The stationary solution of the resulting linear equation can then be solved by using $\phi(\xi) \sim \exp [-\lambda \xi]$, yielding the relation

$$
v(\lambda)=\lambda+\frac{1}{\lambda}
$$

between $v$ and $\lambda$. The curve for the dispersion relation between $v(\lambda)$ and $\lambda$ is

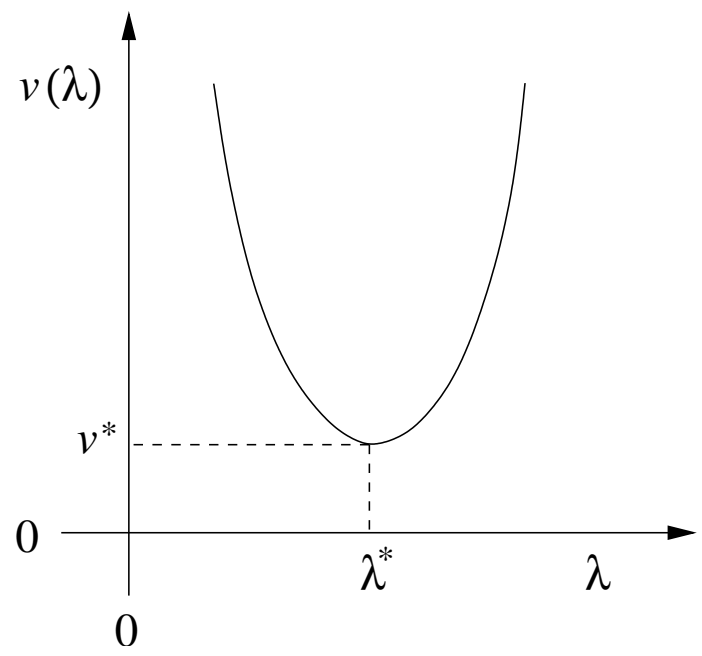

Fig. 2. The dispersion relation (3) is schematically shown above.

schematically shown in Fig. 2. It has a minimum at $\lambda^{*}$, and $v^{*}=v\left(\lambda^{*}\right)=2$.

For any front propagating into a linearly unstable state, there is a solution of the leading edge of the form $\exp [-\lambda \xi]$, and consequently, there exists a dispersion relation between $\lambda$ and the front speed $v(\lambda)$. Of course, the actual dispersion relation depends on the model that one studies. However, irrespective of the model, the universality of pulled fronts lies in the fact that although 
Fig. 2 indicates that one has a front solution for all values of $\lambda$ (and correspondingly all possible front speeds), from which it might a priori seem that the quantities $\lambda^{*}$ and $v^{*}$ are not special in any way, the actual selection of the asymptotic front speed is obtained only after a proper stability analysis of the front profile in the comoving frame. Such a stability analysis yields the result that with an initial condition that $\left.\phi(x, t)\right|_{t=0}$ that decays faster than $\exp \left[-\lambda^{*} x\right]$ for $x \rightarrow \infty^{5}$, for long times, the front speed converges uniformly ${ }^{6}$ to $v^{*}$ as $[13,38]$

$$
v(t)=v^{*}-\frac{3}{2 \lambda^{*} t}+\mathcal{O}\left(t^{-3 / 2}\right)
$$

as the front shape relaxes to its asymptotic configuration $\phi^{*}\left(x-v^{*} t\right)$. However, if the initial shape of the front is such that the leading edge is given by $\left.\phi(x, t)\right|_{t=0} \sim \exp [-\lambda x]$ for $x \rightarrow \infty$ with $\lambda<\lambda^{*}$, then the front speed $v$ remains fixed at $v(\lambda)$, given by the dispersion relation, while its shape remains unchanged at $\exp [-\lambda(x-v t)]$.

(a) $\lambda>\lambda^{*}$

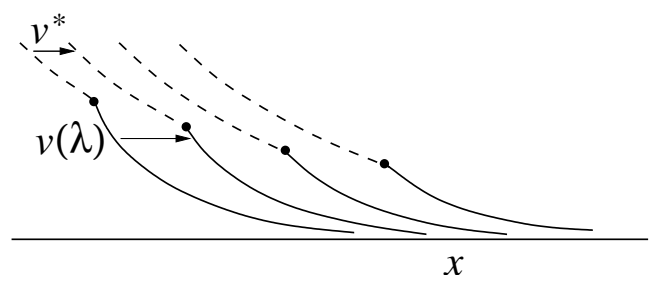

(b) $\lambda<\lambda^{*}$

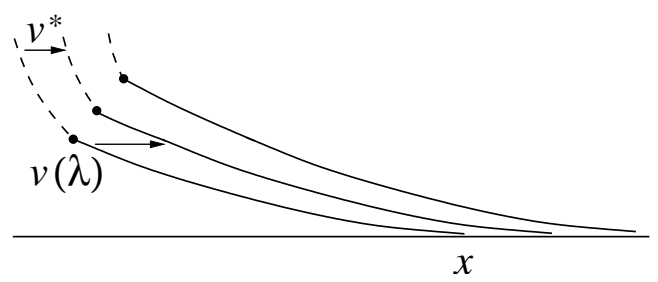

Fig. 3. An intuitive illustration of front speed selection: (a) for $\lambda>\lambda^{*}$, and (b) for $\lambda<\lambda^{*}$. The dashed and the solid profile propagate with their respective speeds. The kink (or the discontinuity in slope is only a symbolic representation of the crossover region [100]. A more precise analysis can be found in Sec. 2 of Ref. [102].

Although obtaining the power law (4) is mathematically quite involved, a beautiful qualitative illustration of the different speed selection behaviour of a pulled front, for $\lambda<\lambda^{*}$ and $\lambda>\lambda^{*}$ with an initial configuration of the leading edge $\phi(x, t=0) \sim \exp [-\lambda x]$ as $x \rightarrow \infty$, has been provided in Ref. [100] (a more precise analysis can be found in Sec. 2 of Ref. [102]). Imagine that we start with an initial configuration of the leading edge, shown in the leftmost curve of Fig. 3(a): an exponential decay $\exp [-\lambda x]$ with $\lambda>\lambda^{*}$ on the right denoted by the solid curve, and the exponential decay $\exp \left[-\lambda^{*} x\right]$ on the left denoted by the dashed curve. The two curves are joined together at a kink shown by a small filled circle. One should keep in mind that a kink in the initial front profile is not propagated as it is for fronts that involve higher than first

5 this condition is also known as "sufficiently steep initial condition".

6 Uniform convergence means that the convergence behaviour (4) of the front speed is the same irrespective of the value of $\phi$ at which the speed is being measured. 
order spatial derivative, but nevertheless, one can get a clear intuitive picture of the evolution of the leading edge by evolving the solid curve $\exp \left[-\lambda^{*} x\right]$ and dashed curve $\exp [-\lambda x]$ profile separately and by following the position of the kink in a pictorial representation. The U-shape of the dispersion relation $v(\lambda)$ vs. $\lambda$ dictates that the speed of dashed curve profile $v(\lambda)$ is higher than the solid curve profile $v^{*}$, as shown in three later snapshots of the evolving leading edge, taken at times $t_{2}<t_{3}<t_{4}$. As a result of the differences in speeds of the dashed and the solid curves, the height of the kink from the $x$-axis keeps decreasing, which shows that front profile at the leading edge is being taken over by the dashed curve, corresponding to an exponential decay with exponent $\lambda^{*}$. A similar picture is shown in Fig. 3(b), where the solid and dashed curves correspond to $\exp [-\lambda x]$ and $\exp \left[-\lambda^{*} x\right]$ respectively, and $\lambda<\lambda^{*}$. The situation is reversed in this case - the front profile at the leading edge is being taken over by the solid curve profile. ${ }^{7}$

The discussions below Eq. (3), so far, is relevant only with an underlying understanding that we are considering a front (propagating into a linearly unstable state) that asymptotically yields a pulled front. In that sense, the usage of Fisher-Kolmogorov equation as an example is very well-placed, since it asymptotically admits only a pulled front solution. As for the question whether an equation describing a propagating front into an unstable state gives rise to a pulled front or not, a necessary condition is not known, but for a sufficient condition, it is known that if all the nonlinear terms in the time evolution of the front suppress growth for a front that propagates into a linearly unstable state, then the resulting front is a pulled front. In particular, for the nonlinear diffusion equation $\partial_{t} \phi=D \partial_{x}^{2} \phi+f(\phi)$ (an equation that has been extensively studied in the front propagation literature), the above sentence implies that one can expect pulled fronts if $f(u) / u \leq f^{\prime}(0)[102]$.

A similar general analysis [i.e., Eqs. (2-3) and related discussions] for the asymptotic speed selection mechanism for pushed fronts does not exist. This is not a surprise in itself, as for pushed fronts, the front speed is really determined in the nonlinear bulk phase of the front (which one cannot solve except for special sets of parameter values). As a result, the leading edge of the front does not play any role in the front speed selection. Nevertheless, it is known that for sufficiently steep initial conditions, as opposed to the $1 / t$ convergence to the asymptotic front speed $v^{*}$ for pulled fronts, the convergence to asymp-

7 The discussion in the above two paragraphs may, at first glance, seem to downplay any special significance of $v^{*}$ - after all, any other front speed $>v^{*}$ can be reached with an appropriately chosen $\lambda$ in the initial configuration of the front. However, in most physical situations, one is interested in the dynamics of "localized initial conditions", for which the spatial decay of the leading edge is steeper than that of $\exp \left[-\lambda^{*} x\right]$. From this point of view, the $\exp [-\lambda x]$ (with $\left.\lambda<\lambda^{*}\right)$ initial configuration of the leading edge, which leads to the eventual front speed $v(\lambda)$, is very special, and therefore is not interesting. 
totic front speed $v^{\dagger}$ for pushed fronts ${ }^{8}$ is exponential in time. For the front speed, a more mathematical representation of the exponential convergence for pushed fronts vs. the non-exponential convergence for pulled fronts can be traced to the stability criteria of the asymptotic front solutions [37, 101, 102]. The idea is that at long times, an intermediate front profile evolving towards its final asymptotics can be decomposed as the asymptotic front profile and infinitesimal localized perturbations around it. The convergence properties to the asymptotic front speed is then determined by how fast, in the comoving frame of the asymptotic front profile, these infinitesimal perturbations decay in time.

To elucidate the relation between the stability of the asymptotic pulled or pushed front solutions and their convergence (in time) behaviour, let us consider a front solution $\Phi_{v}(\xi)$ propagating with a speed $v$, and decompose an intermediate front profile $\phi(x, t)$ as

$$
\phi(\xi, t)=\Phi_{v}(\xi)+\eta(\xi, t)
$$

such that $\eta(\xi, t)$ is of infinitesimal magnitude everywhere. At the leading order, the dynamics of $\eta(\xi, t)$ is described by ${ }^{9}$

$$
\frac{\partial \eta}{\partial t}=\mathcal{L}_{v} \eta+\mathcal{O}\left(\eta^{2}\right)
$$

where the linear operator $\mathcal{L}_{v}$ is obtained by linearizing the equation for $\phi(\xi, t)$ in the comoving frame of the asymptotic solution. ${ }^{10}$ In Eq. $(6), \eta(\xi, t)$ can be expanded as a linear combination of the eigenfunctions of the operator of $\mathcal{L}_{v} .{ }^{11}$ In this expansion, there is an eigenfunction with zero eigenvalue that corresponds to the translational invariance of the asymptotic front solution, while the nature of its lowest-lying eigenvalues decides the decay properties of time convergence properties of the asymptotic front speed [37,101]. For pushed fronts with asymptotic front speed $v^{\dagger}$, the eigenspectrum of $\mathcal{L}_{v^{\dagger}}$ for these lowest-lying eigenfunctions is gapped - this indicates that the convergence to the asymptotic front solution $\phi^{\dagger}\left(x-v^{\dagger} t\right)$ is exponential in time. On the other hand, for pulled fronts, the spectrum of $\mathcal{L}_{v^{*}}$ is gapless, indicating that the convergence to the corresponding asymptotic front solution $\phi^{*}\left(x-v^{*} t\right)$

\footnotetext{
8 Just like $v^{*}$ is a standard notation for the linear spreading speed, $v^{\dagger}$ is also a standard notation for the asymptotic speed of pushed fronts.

9 In this example, we use an equation that involves only a first order derivative in time. A similar analysis can also be worked out for higher time derivatives.

${ }^{10}$ E.g., for Eq. (1), $\mathcal{L}_{v}=D \partial_{\xi}^{2}+v \partial_{\xi}+\left(1-\Phi_{v}^{2}\right)$.

${ }^{11}$ In general, the operator $\mathcal{L}_{v}$ is not a Hermitian operator, and therefore its left and right eigenvectors are different.
} 
has to be non-exponential in nature [101]. ${ }^{12}$

\subsection{Fluctuating (and propagating) Fronts: A Separate Paradigm}

From the point of view that the thermal fluctuations are rather small for the pattern forming systems that relate to the inherent non-equilibrium nature of the bulk phases, it is less of a surprise that the effect of fluctuations on propagating fronts has not attracted the attention of physicists until relatively recently. The understanding that the fluctuations are indeed important was not only motivated by the realization that matter is composed of discrete particles, but also from the fact that there are situations, where fronts are naturally made of discrete constituents on a lattice (see for example, Refs. $[16,28,55,62,96,118,119,124])$. In addition, it is also of general interest to see the effect of externally added noise on the otherwise deterministic fronts, to see how severely this properties are affected, or if these noise terms give rise to new phenomena. Although in some cases, studies concerning the effect of externally added fluctuations on the fronts were motivated by such theoretical interests, e.g. in Refs. $[3,4,79,94,97,99,105,107,108,115]$, in some others, the noise terms were added to model the effect of discreteness of constituent particles and the lattice, e.g. in Refs. [35, 36,67,70,95]. In this review article, we will review both of these cases. In Sec. 2, our focus will be on propagating fronts made of discrete particles on a lattice, there we will review all the known results of such fronts. In Sec. 3, we will review propagating fronts where fluctuations are introduced by means of externally added noise terms. The discussion regarding to what extent the externally added fluctuations correspond to discrete particle models will be discussed subsequently.

The presence of fluctuations, be it as a result of discreteness of particles and the lattice, or be it as a result of the externally noise terms to otherwise deterministic equations, immediately implies that (i) at a contrast to the deterministic fronts propagating with a fixed shape at a fixed speed $v_{\text {as }}$ at $t \rightarrow \infty$, strictly speaking, different snapshots of one particular realization of a fluctuating front, taken at different times, will be microscopically different from each other; although in an average sense, each of these realizations will have the same well-defined shape. The consequences of this is the following: unlike the deterministic fronts, the front position defined by the location $x_{0}$ of the point, where the number of particles per correlation volume is a fixed number, say $N_{0}$ (or by the point where the front field reaches a fixed value $\phi_{0}$ ), does not move

\footnotetext{
$\overline{12}$ The continuous spectrum of $\mathcal{L}_{v^{*}}$ for pulled fronts is known to be responsible for the breakdown of the so-called solvability analysis; for a detailed study, see Ref. [37]. Moreover, the continuity of the spectrum of $\mathcal{L}_{v^{*}}$ is a mathematical representation of the so-called marginal stability criterion of pulled fronts, see for example Refs. $[12,22,23,37,92,101,102]$ etc. and references cited therein.
} 
with a constant speed for a given realization of a fluctuating front even for large times. It is only when the movement of this point over a long period of time is considered (at large times), the speed of this point, calculated as a long time average, can then be defined as the asymptotic front speed. ${ }^{13}$ (ii) Moreover, just like any stochastic quantity having a variance around its average, if one follows $x_{0}$ as a function of time, then one also expects $\left\langle\left[x_{0}(t)-v_{\text {as }} t\right]^{2}\right\rangle$ to increase over time. The angular brackets denote an averaging over an ensemble, each individual member realization of which has reached their steady shapes in an average sense. This means that the displacements of individual front realizations w.r.t. each other as a whole front keeps increasing with time - this a phenomenon known as the wandering of fluctuating fronts.

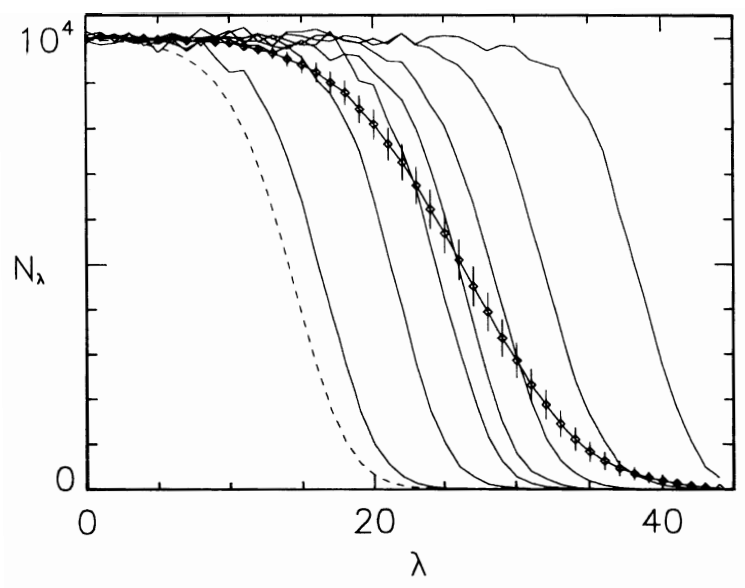

Fig. 4. Adopted from Ref. [16], this figure depicts the front shapes for the reaction-diffusion process $\mathrm{X} \leftrightharpoons 2 \mathrm{X}$ for discrete particles on a lattice, indexed by $\lambda$. The rate of forward reaction $\mathrm{X} \rightarrow 2 \mathrm{X}$ is $N / 2$ with $N=10^{4}$, while rate for the backward reaction $2 \mathrm{X} \rightarrow \mathrm{X}$ has been normalized to unity. Particles diffuse to their nearest neighbour sites with a unit rate. Solid curves: front profiles for seven different realizations at a certain time. Solid curve with error bars: front profile obtained by averaging over number of particles on each lattice site for 66 realizations.

An example of the phenomena in (i) and (ii) of the previous paragraph for fluctuating fronts is shown in Fig. 4. It has been adopted from Ref. [16], and it depicts the shapes of fronts made of discrete particles in a reactiondiffusion process $\mathrm{X} \leftrightharpoons 2 \mathrm{X}$ on a lattice indexed by $\lambda$. The rate of forward reaction $\mathrm{X} \rightarrow 2 \mathrm{X}$ is $N / 2$ with $N=10^{4}$, while the rate for the backward reaction $2 \mathrm{X} \rightarrow \mathrm{X}$ has been normalized to unity. The seven solid curves are the front shapes obtained by taking simultaneous snapshots of seven different realizations at a certain time. All these realizations started with the same initial microscopic configuration. At a microscopic level, the shapes of these snapshots are different, but in an average sense, each of them has a shape

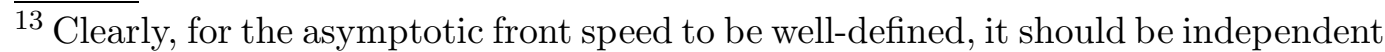
of $N_{0}$ (or $\phi_{0}$ ), which in itself is a consequence that in an average sense, the front realization has a well-defined shape.
} 
identical to that of the dashed curve. The displacements of the individual front realizations as a whole w.r.t. each other demonstrate front wandering. On the other hand, the solid line with error bars in it depicts the front shape obtained by averaging over number of particles on the lattice sites for 66 different front realizations. As one can expect, the front obtained by such an averaging process is much wider than any of the seven realizations, since such an averaging fails to filter out the effect of front wandering - in future, we will refer to this averaging process as "simple averaging".

The above discussion, therefore, points us to the following conclusions: (a) to obtain the average asymptotic shape of fluctuating fronts, one has to separate out the wandering effects, i.e., one has to first realign the simultaneous realizations and then calculate the average shape - from now on, we will refer to such an averaging as "conditional (ensemble) averaging", ${ }^{14}$ and $\left\langle\left\langle N_{k}\right\rangle\right\rangle$ will denote the conditionally averaged number of particles in the $k$-th lattice site. (b) For measuring the front speed, a conditional averaging is not necessary; one can obtain the front speed by tracking how fast a given value of the simply averaged front profile $\phi$, say $\phi_{0}$, moves. However, we have seen before, for deterministic fronts, that the front shape and the speed are related to each other, and therefore, from that angle, for a theoretical prediction/analysis of front speed, one does need the conditionally averaged front profile. A good description of fluctuating fronts must take both (a) and (b) into account. In addition, (c) a comprehensive description of fluctuating fronts must not only include the expression of the asymptotic front speed and as well as its approach in time towards a steady (conditionally averaged) front shape starting from an initial configuration, but also must it identify the nature of its wandering (i.e., whether it is diffusive, sub- or super-diffusive), along with the relevant characterizing exponents. ${ }^{15}$ Such a comprehensive description for fluctuating fronts propagating into unstable states is the central theme of this review article. In one spatial dimension, our convention will be to consider fronts propagating from the left to the right, and keeping in line with the propagation direction of the front, we will often use forward for rightward and backward for leftward interchangeably.

Although I claim to provide a comprehensive description for fluctuating fronts in this review article, its precise meaning has to be properly laid out at this point - after all, a front is nothing but an interface that demarcates the boundary between two different phases within a system; and the properties of

\footnotetext{
${ }^{14}$ It is precisely this conditionally averaged front profile that is described by an appropriate deterministic mean-field equation; in future, these conditionally averaged front profile will be denoted by $\phi^{(0)}$. To find out how to align different snapshots of fluctuating fronts in the sense of (a) above, see Eq. (78) and footnote 65. For now, the mere assumption that $\phi^{(0)}$ exists will be enough.

${ }^{15}$ In one spatial dimension, fluctuating fronts are always diffusive; in higher dimensions, the situation is more complicated.
} 
such interfaces for, e.g., fluid flows in porous media, flame fronts in a burning paper, atom deposition processes in molecular beam epitaxy experiments, aggregate fronts in diffusion-limited-aggregation etc. have already been very well-studied in the literature. Analyzing the properties of fronts in such vastly different systems is by no means the intention of this review. Instead, my purpose here is the following: in spite of the existence of a large variety of dynamical equations that describe moving interfaces in different systems, there is an overall underlying framework for the propagation mechanism of deterministic fronts; thus, I want to review the effect of fluctuations on propagating fronts under the same framework in a comprehensive manner.

In general, providing such a comprehensive description for fluctuating fronts is by no means an easy task. As mentioned in the first paragraph of Sec. 1.2, two major approaches have been used to this end - the first one being that of noisy dynamical equations, where one simply adds external noise terms to the otherwise deterministic equations to introduce fluctuations. In the second approach, many researchers in the last few years have taken the route of studying fluctuating fronts that are constituted of discrete particles on a lattice, where stochasticity of the microscopic dynamics of the particles themselves gives rise to fluctuations. In a way, the philosophy of both these approaches is to first have a deterministic front as the underlying structure, and then to study the effect of fluctuations superimposed thereupon: there exists a parameter in these approaches that controls the fluctuation strength. For example, in the second approach, this parameter is $1 / N$, where $N$ is the conditionally averaged number of particles per lattice site in the stable phase of the front, and the deterministic mean-field results are obtained when $N \rightarrow \infty$. In the recent past, there has also appeared a new approach (along the lines of quite the opposite philosophy of the two approaches discussed above) to extract the mean-field behaviour at small $N$ [81]. Therein, one defines a variable $N^{*}$ that measures the number of particles per correlation volume in the stable phase of the front, and depending on the system parameters, one can have $N^{*} \rightarrow \infty$ for correlation volume $\rightarrow \infty$ and small $N$; in this limit, one also recovers the deterministic mean-field description for the front.

Nonetheless, it has to be noted that the majority of the studies on fluctuating fronts have been carried out on fluctuating "pulled" 16 fronts, i.e., on fronts whose deterministic limit yield pulled fronts, as opposed to on fluctuating pushed fronts ${ }^{17}$. This is not very surprising — first of all, for the deterministic counterparts, it is the pulled fronts, for which there is a solid theoretical

\footnotetext{
$\overline{16}$ The use of the quotes is motivated by the fact that fluctuating fronts, whose deterministic limit yield pulled fronts, are actually weakly pushed. See points IV and V of Sec. 2.1 for more details.

${ }^{17}$ In view of footnote 16 , what we mean by fluctuating pushed fronts is that the deterministic limit of these fronts yield pushed fronts.
} 
understanding. Secondly (and more importantly), it is the sensitivity of the pulled fronts to the dynamics of the leading edge, which is severely affected by the discreteness of particles and the lattice, or by the presence of external noise terms - as a result, fluctuating "pulled" fronts exhibit many surprising characteristics. 


\subsection{A Note on the Notations}

Before we proceed further on, it will be very helpful to have the convention of the notations used summarized. Below we provide a table for this purpose. Any notation that does not appear in Table I is explained before its usage in this review article.

\begin{tabular}{||l|l||}
\hline \hline$x, k$ & Notations for continuum space and for lattice index respectively. \\
\hline$v^{*}$ & $\begin{array}{l}\text { Notation for the linear spreading speed for deterministic fronts } \\
\text { propagating into unstable states, and also for the selected asymp- } \\
\text { totic front speed for (deterministic) pulled fronts with a steep } \\
\text { enough initial condition. Has already been introduced in Sec. 1.1. } \\
\text { Applies to deterministic pulled fronts both in continuum space and } \\
\text { on a lattice. }\end{array}$ \\
\hline$v^{\dagger}$ & $\begin{array}{l}\text { Notation for the selected asymptotic front speed for (determin- } \\
\text { istic) pushed fronts. Also introduced already in Sec. 1.1. Applies } \\
\text { to deterministic pushed fronts both in continuum space and on a } \\
\text { lattice. }\end{array}$ \\
\hline$v_{\text {as }}$ & $\begin{array}{l}\text { General notation for the asymptotic front speed of any front } \\
\text { (pulled and pushed) in any system (deterministic and stochastic; } \\
\text { discrete and as well as continuum space). }\end{array}$ \\
\hline$v_{N}$ & $\begin{array}{l}\text { Notation for front speed for a discrete particle and lattice system } \\
\text { of fronts, where there are } N \text { particles on (conditional) average per } \\
\text { lattice site. }\end{array}$ \\
\hline$\xi$ & $\begin{array}{l}\text { Notation for comoving co-ordinate for pulled }\left(\xi=x-v^{*} t, \text { or }\right. \\
\left.\xi=k-v^{*} t\right), \text { or fluctuating "pulled" fronts }\left(\xi=k-v_{N} t\right) \text { or even } \\
\text { for fronts whose deterministic mean-field limit yield pulled fronts } \\
\text { but whose speeds have not been determined yet (in these cases, } \xi \\
\text { will be used to denote } x-v_{\text {as }} \text { or } k-v_{\text {as }} t \text { or } k-v_{N} t \text { as appropriate). }\end{array}$ \\
\hline$\zeta$ & $\begin{array}{l}\text { Notation for comoving co-ordinate for pushed }\left(\zeta=x-v^{\dagger} t, \text { or }\right. \\
\left.\zeta=k-v^{\dagger} t\right), \text { or fluctuating pushed fronts }\left(\zeta=k-v_{N} t\right), \text { or even } \\
\text { for fronts whose deterministic mean-field limit yield pushed fronts } \\
\text { but whose speeds have not been determined yet (in these cases, } \zeta \\
\text { will be used to denote } x-v_{\text {as }} \text { or } k-v_{\text {as }} t \text { or } k-v_{N} t \text { as appropriate). }\end{array}$ \\
\hline$\phi^{*}(\xi)$ & $\begin{array}{l}\text { Notation for the front profile for pulled fronts; either } \xi=x-v^{*} t \\
\text { or } \xi=k-v^{*} t \text {. Already introduced in Sec. } 1.1 .\end{array}$ \\
\hline$\phi^{\dagger}(\zeta)$ & $\begin{array}{l}\text { Notation for the front profile for pulled fronts; either } \zeta=x-v^{\dagger} t \\
\text { or } \xi=k-v^{\dagger} t . \text { Already introduced in Sec. } 1.1 .\end{array}$ \\
\hline \hline
\end{tabular}

Table I: Convention behind the notations used in this review article. 


\section{Fluctuating Fronts in Discrete Particle and Lattice Systems}

\subsection{Summary of Known Results}

In Sec. 2.1, we summarize all the known results for fluctuating (propagating) fronts, made of discrete particles on a lattice. Among the physical systems, where front propagation has been studied under this scheme, various sorts of reaction-diffusion systems constitute the majority $[5,14,16,34,54-56,66$, $70,71,73,80,86,87,96,116,118,119]$. In addition, propagating fronts were also studied in the context of directed polymers in random media [19-21,24,27,28], and the calculation of the largest Lyapunov exponent in a gas of hard spheres [122-126]. In all these models, fronts propagate from a stable to an unstable state. The unstable state is constituted of empty lattice sites, whereas in our notation, the (conditionally) averaged number of particles on the $k$-th lattice site $\left\langle\left\langle N_{k}\right\rangle\right\rangle$ in the stable state and the corresponding asymptotic front speed are respectively denoted by $N$ from now on. Also, unless otherwise stated, in Sec. 2, fronts will be assumed to propagate in one spatial dimension; fronts in higher than one spatial dimensions will be considered only in Sec. 2.6.6.

For these models, the following results have been obtained in the last decade:

I. The limit of deterministic pulled front propagating with speed $v^{*}$ is reached for $N \rightarrow \infty$. However, the convergence of $v_{N}$ to its deterministic limit $v^{*}$ for increasing value of $N$ is extremely slow. The approach of $v_{N}$ to $v^{*}$ is from below, and for asymptotically large $N$ values, the leading order form of $v_{N}$ is model independent [19-21,56,86,87,122,124-126], given by

$$
v_{N}=v^{*}-\left.\frac{d^{2} v(\lambda)}{d \lambda^{2}}\right|_{\lambda^{*}} \frac{\pi^{2} \lambda^{* 2}}{\ln ^{2} N} .
$$

II. For intermediately large values of $N$, the subdominant corrections to the leading order result (7) are very strong, and they depend on the details of the model. For some models, these corrections can be so strong that one does not observe the asymptotic scaling (7) unless $N$ is extremely large $[16,56,66,70,86,87]$.

III. Unlike the semi-infinite leading edge for deterministic pulled fronts, in any snapshot of these discrete particle and lattice systems for any value of $N$ at any time, there exists a "foremost occupied lattice site (f.o.l.s.)", on the right of which no lattice site has ever been occupied before. In the hopes of providing a theory that would yield front speeds at intermediately large values of $N$ for these fronts, a stochastic dynamics of the f.o.l.s., and a (standard) deterministic and uniformly translating front solution reasonably 
far behind has been proposed in Ref. $[86,87]$. The two solutions are then matched in the "tip region" of the front, spatially extended over a few lattice sites behind the f.o.l.s. (and also including the f.o.l.s.). Despite the fact that this formalism yields a consistent (and verifiable) picture of these fronts, it is not predictive, and a first-principle based predictive theory for $v_{N}$ at intermediately large values of $N$ is still lacking.

IV. Unlike pulled fronts, where the asymptotic front speed $v^{*}$ is reached only if the initial front profile decays faster than $\exp \left[-\lambda^{*} x\right]$ for $x \rightarrow \infty$, it can be argued that the conditionally averaged front profile for fluctuating "pulled" fronts is reached uniquely, independent of the initial microscopic configuration. This observation has been made in Ref. [21], and it is consistent with all the known simulation results so far.

V. In the limit of $N \rightarrow \infty$, the spectrum of the stability operator $\mathcal{L}_{v_{N}}$ [c.f. Eq. (6)] is gapped, and for the lowest-lying eigenvalues, the gap is $\propto 1 / \ln ^{2} N[56,89]$. Although we will get into more details later on, here we briefly mention that points IV and V together bear the signature of these fronts being weakly pushed.

VI. In the limit of $N \rightarrow \infty$, the diffusion coefficient of the front $D_{f}$ that characterizes front wandering, approaches zero. For fluctuating "pulled" front in a model that closely resembles the so-called clock model [124], $D_{f}$ has been shown to scale as $1 / \ln ^{3} N$ in the large $N$ asymptotic regime [21].

VII. As $N$ is reduced "too much", the fluctuations in the number of particles per lattice site in the stable state become stronger and stronger. Perhaps in view of II above, in the sense that due to the lack of first-principle based predictive theory for finite- $N$ corrections, works on such values of $N$ are somewhat rare $[71,118,119]$. The extreme limit of this, namely the case when there is at most one particle is allowed per lattice site, however, is very well studied (we will refer to this scenario as " $N \leq 1$ "). In view of the fact that there are simply too many results to summarize here, the readers are referred directly to Sec. 2.6 for further details.

VIII. As far as the discrete particle (and lattice) systems of fluctuating pushed fronts are concerned (c.f. footnote 17), at the time of writing this review article, I have not seen any work on the behaviour of $v_{N}$ for $N \rightarrow \infty$. Nevertheless, having combined the knowledge that one musters from points I through VI above and the solvability analysis for pushed fronts [37], for $N \rightarrow \infty$, the convergence of the asymptotic front speed to $v^{\dagger}$ (c.f. footnote 8) for fluctuating pushed fronts to their deterministic limit behaves as a power law of $N$.

In the rest of Sec. 2, we will elaborate points I-VIII, and provide a unified picture of discrete particle systems of fluctuating fronts. Details of which point 
is elaborated where are given below: point I in Sec. 2.2, points II and III in Sec. 2.3, points IV and V in Sec. 2.4, point VI in Sec. 2.5, point VII in Sec. 2.6, and point VIII in Sec. 2.7. Finally, in Sec. 2.8 we put the results of Secs. $2.2,2.4-2.5$ and 2.7 in a unified perspective.

\subsection{Derivation of $1 / \ln ^{2} N$ Convergence of the Asymptotic Front Speed to $v^{*}$ for Fluctuating "Pulled" Fronts as $N \rightarrow \infty$}

From its definition in Sec. 1.1, pulled fronts can be clearly seen to be realized in a continuum description, for otherwise the "infinitesimal perturbation around the unstable state" does not quite make sense. Through its definition (and propagation mechanism), pulled fronts and infinitesimal perturbations around the unstable state are intimately intertwined — ahead of the leading edge of a pulled front, the perturbation around the unstable state is infinitesimal, as it grows and propagates itself with speed $v^{*}$ and pulls the rest of the front along with it. From this perspective, the discrete particle and lattice systems of fluctuating "pulled" fronts belong to a different category - at the leading edge of these fronts, due to discreteness of the particles, the smallest amount of the "infinitesimal perturbation" for growth to start on any lattice site cannot be less than that of one quantum of particle, a fact that is more than likely to yield a front speed different from $v^{*}$.

In hindsight, the above argument sounds simple, and it is appreciated easily when the conditionally averaged number of particles per lattice site is translated into the language of $\phi$ of Sec. 1.1. Notice that in the stable phase of the front, $\left\langle\left\langle N_{k}\right\rangle\right\rangle=N$ corresponds to $\phi=1$. As a result, the lowest amount of $\phi$ corresponding to one quantum of particle, needed on any lattice site ahead of the leading edge for growth to begin is $1 / N$. The pulled front limit in these discrete particle systems is therefore expected to be reached only for $N \rightarrow \infty$.

These subtleties were first realized by Brunet and Derrida [19], shortly after simulations revealed that for models of fluctuating "pulled" fronts the deviations of the asymptotic front speed from $v^{*}$ can be significant [16], even for $N \gg 1{ }^{18}$ To mimic the lowest quantum of particle that is needed on any lattice site ahead of the leading edge for the growth to begin, Brunet and Derrida conjectured that the dynamics of a fluctuating "pulled" front at large $N$ should be described by an equation with a growth cutoff for $\phi \leq \varepsilon$, where $\varepsilon=1 / N$.

To verify their ideas, they used modified Eq. (1):

\footnotetext{
${ }^{18}$ Severe deviations of the front speeds from $v^{*}$ for $N$ not so larger than 1 have also been observed in simulations, but we leave those aside until we discuss point VII at length in Sec. 2.6.
} 


$$
\frac{\partial \phi}{\partial t}=\frac{\partial^{2} \phi}{\partial x^{2}}+\left(\phi-\phi^{n}\right) \Theta(\phi-\varepsilon) .
$$

The stationary front solution of Eq. (8), propagating with speed $v_{\varepsilon}$, was then divided into three regions: I, II and III. In region II and III, $\phi \ll 1$ and hence the nonlinear term $\phi^{n}$ can be ignored; in addition, in region III, $\phi<\varepsilon$, which leads to a further simplification of Eq. (8). On the other hand, in region I, one has to consider the full nonlinear equation. This leads one to

$$
\begin{aligned}
-v_{\varepsilon} \frac{d \phi}{d \xi} & =\frac{d^{2} \phi}{d \xi^{2}}+\phi-\phi^{n} & & \text { in region I, } \\
-v_{\varepsilon} \frac{d \phi}{d \xi} & \simeq \frac{d^{2} \phi}{d \xi^{2}}+\phi & & \text { in region II, and } \\
-v_{\varepsilon} \frac{d \phi}{d \xi} & =\frac{d^{2} \phi}{d \xi^{2}} & & \text { in region III . }
\end{aligned}
$$

At the boundary between regions II and III, the continuity of the value of the field $\phi$ as well as of its comoving derivative $\phi^{\prime}(\xi)$ has to be maintained. With the understanding that $\varepsilon \ll 1$, the leading order solutions that obey these boundary conditions were shown to be $[19]^{19}$

$$
\begin{aligned}
\phi^{(0)}(\xi) & =\varepsilon e^{-v_{\varepsilon}\left(\xi-\xi_{0}\right)} & \text { in region III, and } \\
& =\frac{A}{\pi \lambda^{*}}|\ln \varepsilon| \sin \left[\frac{\pi \lambda^{*}\left(\xi-\xi_{1}\right)}{|\ln \varepsilon|}\right] e^{-\lambda^{*}\left(\xi-\xi_{1}\right)} & \text { in region II, }
\end{aligned}
$$

such that the boundary between regions II and III lies at $\xi_{0} \simeq|\ln \varepsilon| / \lambda^{*}$, whereas the solution in region II crosses over to that in region I at $\xi_{1}$, and $A$ is a quantity of $\mathcal{O}(1)$; yielding

$$
v_{\varepsilon}=v^{*}-\left.\frac{d^{2} v(\lambda)}{d \lambda^{2}}\right|_{\lambda^{*}} \frac{\pi^{2} \lambda^{* 2}}{\ln ^{2} \varepsilon} .
$$

Notice that to obtain the expression (11) of $v_{\varepsilon}$, the solution of Eq. (9) in region I is not required. Moreover, although from solution $(10) \phi^{(0)}(\xi)$ might seem to go to zero at $\xi=0$ violating the monotonicity of the front profile, in reality, there exists no sharp boundary between regions I and II, like the one at $\xi_{0}$. Instead, the solution (10) crosses over to that of region I, and the crossover happens in a way that maintains the monotonicity of the front profile. At the leading order, the characteristic position $\xi=\xi_{1}$ for the crossover is given by $\xi_{1} \simeq 0$.

\footnotetext{
${ }^{19}$ Notice the notation $\phi^{(0)}$ : the idea is motivated by the fact that as $N \rightarrow \infty$, the conditionally averaged front shape is given by the effective deterministic mean-field equation (9). See footnote 14 in this context.
} 
Few points in Brunet and Derrida's conjecture must be noted: (a) One arrives at the leading order large $N$ asymptotic behaviour of the front speed (7) by setting $\varepsilon=1 / N$. The form of $v_{N}$ is independent of the details of the microscopic dynamics of the constituent particles, although the actual dispersion relation $v(\lambda)$ vs. $\lambda$ is obtained from the equation obeyed by the deterministic mean-field limit of the microscopic model at hand. (b) Region III is characterized by an effective (finite) width $1 / v_{\varepsilon}$ due to the exponential decay of the front profile. On the other hand, with the substitution $\varepsilon=1 / N$ for asymptotically large $N$, the length $\left(\xi_{0}-\xi_{1}\right)$ of region II increases logarithmically with $N$, while the length of region I remains finite - this yields an effective front width, scaling as $\ln N$. (c) Furthermore, the leading order form of $v_{N}$ is independent of whether one substitutes $\varepsilon=1 / N$ or $\varepsilon=c / N$, with $c$ of $\mathcal{O}(1)$. In addition to amplitude $A, c$ simply provides a subdominant correction to the r.h.s. of Eq. (7), if $c \neq 1$.

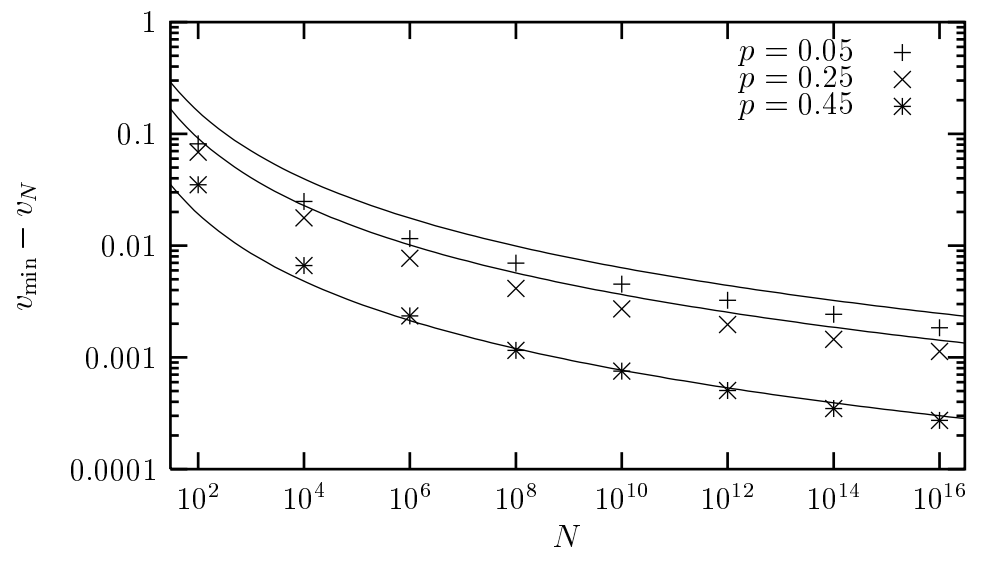

Fig. 5. The plot of $v^{*}-v_{N}$ vs. $N$ for the discrete particle and lattice model of Refs. [19-21] relating to the study of directed polymers in random media. In the above figure, $v_{\min }$ should be read as $v^{*}$. The solid curves represent Eq. (7) for various $p$ values [20]. A corresponding graph for the same model for much higher values of $N$ for $p=0.25$ can be found in Fig. 2 of Ref. [21].

In the history of this subject, Brunet and Derrida's insight has turned out to have had an enormously significant impact. Their prediction of the $N$ dependence of the front speed was immediately confirmed in a fluctuating "pulled" front model (where time was also discretized) [19-21] ${ }^{20}$ relating to directed polymers in random media (see Fig. 5) [24,27]. Subsequently, in another model of a fluctuating "pulled" front - namely, the so-called clock model relating to the calculation of the largest Lyapunov exponent in a gas of hard spheres $[122,124-126]^{21}$ — the idea of a cutoff was employed; and the

$\overline{20}$ The deterministic mean-field limit for this model is described by the equation $\phi(k, t+1)=1-p[1-\phi(k-1, t)]^{2}-(1-p)[1-\phi(k, t)]^{2}$, where $k$ is the lattice site index and $p$ is a fixed number in the interval $(0,0.5)$. The corresponding $\mathrm{U}$-shaped dispersion relation $v(\lambda)$ vs. $\lambda$ has the form $v(\lambda)=\ln \left[2 p e^{\lambda}+2(1-p)\right] / \lambda$.

${ }^{21}$ The deterministic mean-field limit of the so-called clock model is described by 
sinusoidal profile of Eq. (10) at the leading edge, along with the result that $v^{*}-v_{N} \propto 1 / \ln ^{2} N$ for asymptotically large $N$, was obtained (see Fig. 6).

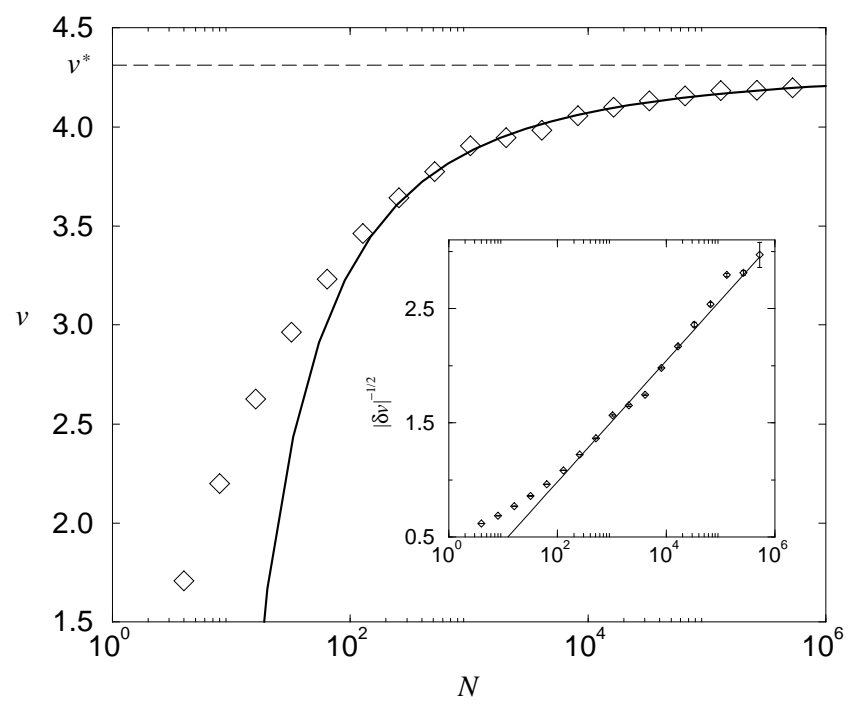

Fig. 6. The plot of $v^{*}-v_{N}$ vs. $N$ for the so-called clock model $[122,124-126]$. Symbols: simulation data; solid curve in the main graph: expression (7); inset: the same data as of the main graph plotted differently, with $\delta v=v^{*}-v_{N}$.

Although a continuum-space equation was used in Ref. [19] for the deviation of Eq. (7), it turns out that for asymptotically large $N$, one can motivate the sinusoidal profile of the leading edge along the lines of the discussion in the first two paragraphs of Sec. 2.2 and the positivity of $\phi$ (in these models $\phi_{k}$ represents the scaled number of particles on the lattice site $k$, and hence it can never be negative) $[86,87,122,125,126]$, from which one arrives at Eq. (7) very simply for any given microscopic model. The idea is the following: since the growth of particles ahead of the leading edge of these fronts does not start unless there is at least one particle on any lattice site, one can expect the discreteness of particles to inhibit the spreading of the leading edge, resulting in a front speed $v_{N}$ smaller than $v^{*}$. However, $v^{*}$ is the minimum value of $v(\lambda)$ for any real $\lambda$ in the deterministic mean-field description of the microscopic model at hand, and therefore the only way to obtain $v_{N}<v^{*}$ from the dispersion relation $v(\lambda)$ vs. $\lambda$ is to consider $\operatorname{Im}(\lambda) \neq 0$.

Equation (10) originates from the consideration of non-zero $\operatorname{Im}(\lambda)$, which enters the solution as the inverse of the wavelength of the sin-function. The positivity of $\phi$ then restricts the value of its argument to lie within $(0, \pi)$ (modulo a constant phase factor, which can be set to zero by shifting the position of the origin in the comoving frame). For $N \rightarrow \infty, \operatorname{Re}(\lambda) \rightarrow \lambda^{*}$, $\operatorname{Im}(\lambda) \rightarrow 0$ and $v_{N} \rightarrow v^{*}$, and as a result, for asymptotically large $N$, one can expand the front solution around these asymptotic values. Such an expansion

$\overline{\frac{\partial \phi_{k}}{\partial t}=}-\phi_{k}+\phi_{k-1}^{2}$, leading to the dispersion relation $v(\lambda)=\left[2 e^{\lambda}-1\right] / \lambda$. 
confines the values of $\operatorname{Re}(\lambda), \operatorname{Im}(\lambda)$ and $v_{N}$ close to the minimum of the dispersion relation $v(\lambda)$ vs. $\lambda$ on the $\operatorname{Re}(\lambda)$-plane, and at the leading order, it yields the following results: (a) $\operatorname{Im}(\lambda)=\ln N / \pi \lambda^{*}$, just like in the argument of the sin-function in Eq. (10), and (b) the large $N$ asymptotics (7), where the second derivative $\left.v^{\prime \prime}(\lambda)\right|_{\lambda^{*}}$ appears due to the fact that near the extremum of $v(\lambda)$ on the $\operatorname{Re}(\lambda)$-plane, the variation of the front speed for a small variation of $\operatorname{Re}(\lambda)$ from $\lambda^{*}$ is $\left.\propto v^{\prime \prime}(\lambda)\right|_{\lambda^{*}}$.

We conclude Sec. 2.2 with a short note on the subdominant corrections to the $\propto 1 / \ln ^{2} N$ deviation of $v_{N}$ from $v^{*}$. As mentioned earlier, according to the derivations in Ref. [19], along with other explicit dependence on $N$, both $A$ and $c$ enter the expression of the subdominant corrections. For asymptotically large values of $N$, the behaviour of the subdominant corrections is dominated by these explicit $N$-dependent terms rather than the ones involving $A$ and c. For the microscopic model relating to directed polymers in random media, a $1 / \ln ^{\beta} N$ form has been suggested for the leading order behaviour of the subdominant corrections in Ref. [21], where $\beta$ has been estimated to lie somewhere between 2.5 and 3 . However, for the reaction-diffusion system $\mathrm{X} \leftrightharpoons 2 \mathrm{X}$ in Ref. [86,87], at the leading order, the subdominant corrections seem to behave as $\ln [\ln N] / \ln ^{3} N$. Although this issue has not yet been settled, it is only a theoretical and a marginal one - after all, at $N$ values when the subdominant corrections start to become stronger, a very different description of fluctuating "pulled" fronts is called for, as we will find out next.

\subsection{The Case of Intermediately Large Values of $N$, Foremost Occupied Lat- tice Site, Tip Region of the Front and All That}

The large $N$ asymptotics (7) describes $v_{N}$ as an explicit function of $N$ at the leading order. However, from comparison with the simulation data, it seems that to observe this asymptotic result, one often has to go to very high values of $N$. In support of their theoretical prediction (7) in Ref. [19], Brunet and Derrida took $N$ up to $10^{16}$ (Fig. 5) $[19,20]$ in the lattice model relating to directed polymers in random media; and in a later work [21], by means of a clever simulation algorithm, they further took $N$ to as high as $10^{150}$ in the same model. In these studies, the range where $N$ is intermediately large but not asymptotically large, was not examined carefully. Amid this backdrop, a reinterpretation of the numerical results of Ref. [16] for the reaction-diffusion system $\mathrm{X} \leftrightharpoons 2 \mathrm{X}$ suggests that there are strong deviations of the data from the prediction (7) when $N$ is of $\mathcal{O}\left(10^{4}-10^{6}\right)$. Such deviations were also noted by Kessler and co-workers [56] — their simulations for fluctuating "pulled" fronts in the reaction-diffusion system $\mathrm{X}+\mathrm{Y} \rightarrow 2 \mathrm{X}$ on a lattice suggested that even for $N$ as large as $10^{12}$, differences between simulation data and the large $N$ asymptotics (7) remain significant (see Fig. 7). In view of these, one's worry 
naturally shifts to the subdominant corrections to the r.h.s. of Eq. (7).

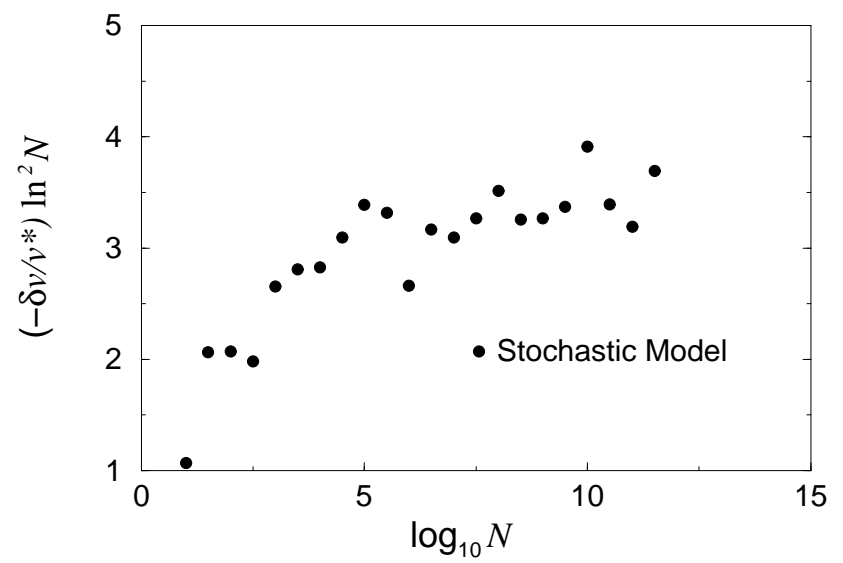

Fig. 7. Simulation data from Ref. [56] for the reaction-diffusion system $\mathrm{X}+\mathrm{Y} \rightarrow 2 \mathrm{X}$. The rate of diffusion $D=0.5$, the reaction rate is $0.1 / N$, and $\delta v=v^{*}-v_{N}$. Note the increasing trend of the data with increasing $N$. For these parameter values, according to Eq. (7), $\delta v \ln ^{2} N / v^{*}$ should be $\simeq-9.6$. The figure is an altered version of Fig. 5 in Ref. [56]. The alteration was carried out for greater clarity.

The common denominator of all the derivations of Eq. (7) $[19,86,87,122,125$, 126] for fluctuating "pulled" fronts is that they were carried out to describe a conditionally averaged uniformly translating profile all over the front. In such a description, for the large $N$ asymptotics, the leading order expression of $v_{N}$ is independent of specific details of the microscopic model. The specific model dependence ${ }^{22}$ of $v_{N}$ enters in the subdominant corrections through two parameters, $A$ and $c(\varepsilon=c / N)$. In that sense, Brunet and Derrida's derivation of Eq. (7) is quite instructive - it shows that the speed of a fluctuating "pulled" front is actually fully determined from the overall shape of the front (thereby taking into account the specific details of the model in a conditionally averaged manner), and not only from the property of the leading edge - after all, to obtain the value of $A$, one needs to know the overall front shape, even in the region where the nonlinearities are important. ${ }^{23}$ On the other hand, although $c$ was set equal to 1 by Brunet and Derrida, one might wonder what its true value is, and to what extent it influences the subdominant corrections

The dependence of $v_{N}$ on the specific details of the model is apparent from a comparison of Figs. 6 and 7. While in Fig. 7, even at $N$ as large as $10^{12}$, the $1 / \ln ^{2} N$ scaling of $v^{*}-v_{N}$ seems rather far off for the reaction-diffusion system $\mathrm{X}+\mathrm{Y} \rightarrow 2 \mathrm{X}$, Fig. 6 finds confirmation of the $1 / \ln ^{2} N$ scaling at $N \sim 10^{3}$ for the so-called clock model.

${ }^{23}$ This stands in sharp contrast to pulled fronts. To obtain the front speed $v^{*}$ for pulled fronts, one only needs the dispersion relation $v(\lambda)$ vs. $\lambda$. The dispersion relation is obtained from the consideration of the leading edge of the front, where the nonlinearities are not important. 
for a given value of $N$ in a microscopic model. ${ }^{24}$ It is in fact conceivable that these parameters have further dependence on $N$; nevertheless, the feasibility of ever obtaining an explicit $N$-dependent prediction of $v_{N}$ for any value of $N[56]$ remains an open question.

In view of these difficulties, if one were to provide a description of fluctuating "pulled" fronts to yield front speeds $v_{N}$ for moderately large values of $N$ as well, one must certainly look elsewhere to address a pressing concern; namely the effect of stochasticity in the microscopic dynamics of the individual particles on the propagating front, an effect that disappears when the conditional averaging of a large number of front realizations is performed all over the front region.

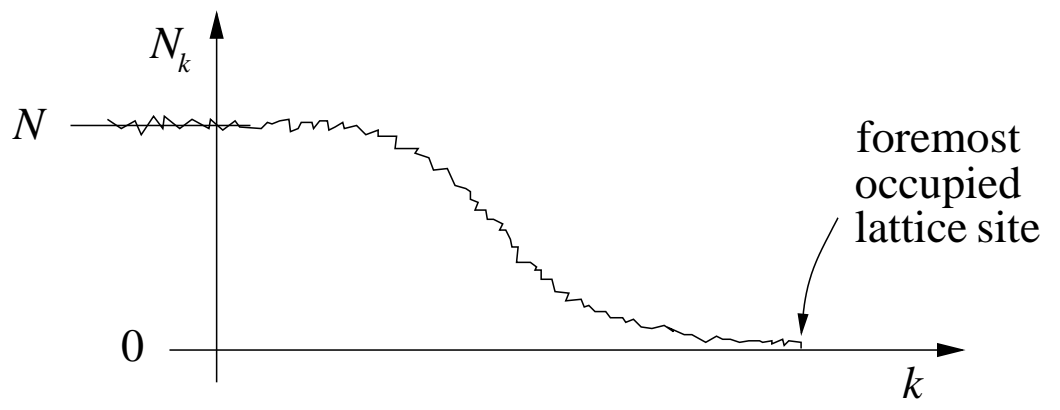

Fig. 8. An instantaneous snapshot of a front realization at large times. Note the existence of the foremost occupied lattice site, on the right of which no lattice site has been previously occupied.

Such a description, put forward in Ref. [86,87], is motivated by the microscopic picture of the front dynamics at the far end of the front. The idea is based on the observation that in every discrete particle and lattice system of a fluctuating front, at all times there exists a foremost occupied lattice site (f.o.l.s.), which is defined as the one, on the right of which no lattice site has ever been occupied before (see Fig. 8). As front propagation in this framework is effectively tantamount to forward (rightward) movement of the f.o.l.s., the crucial role played by the discreteness effects of the particles and the lattice is reflected in the mechanism of the front propagation at the f.o.l.s., where the mechanism is not that of uniform translation, but instead, is of "halt-and-go". A lattice site, which has never been occupied before, attains the status of the f.o.l.s. as soon as one particle hops into it from behind (left). In reference to the lattice, the position of the f.o.l.s. remains fixed at this site for some time, i.e., after its creation, an f.o.l.s. remains the f.o.l.s. for some time. During this time, however, the number of particles on and behind the f.o.l.s. continues to grow, and as a consequence, the chance of one of them making a hop on to

${ }^{24}$ In this context, we draw the reader's attention to the quantity $a$ in the derivation of the large $N$ asymptotics (7) in Ref. [86,87]. The quantities $a$ and $c$ are in fact equivalent, both are introduced by hand, and the values of none of them can be obtained from a first principle derivation. 
the right of the present f.o.l.s. also increases. At some instant, a particle from behind hops over to the right of the present f.o.l.s.: as a result of this hop, the position of the f.o.l.s. advances, or, viewed from another angle, a new f.o.l.s. is created on the right of the present one.

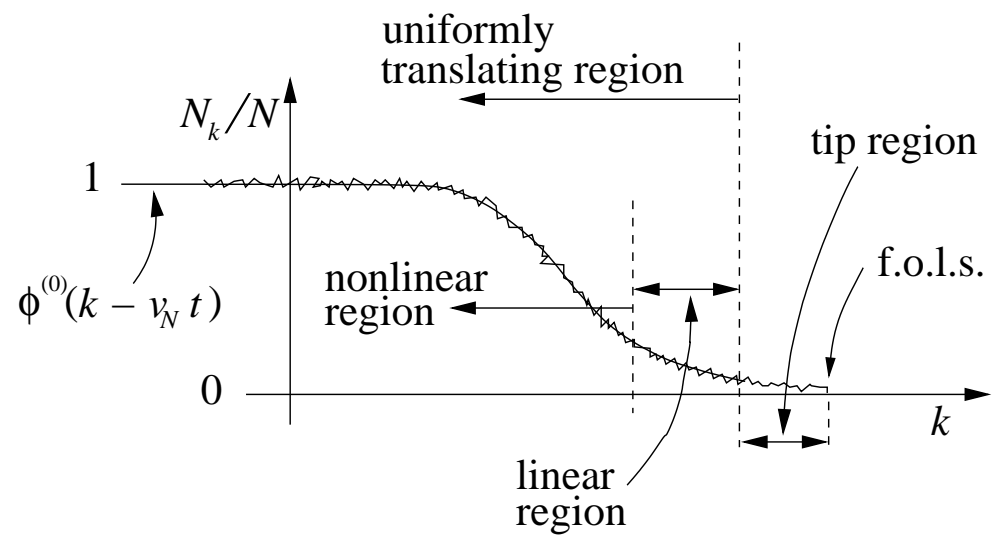

Fig. 9. A snapshot of a realization of a fluctuating front (jaggered curve) at large times, and how a theorist might picture such a front (smooth curve). In this picture, as the smooth curve shows, a uniformly translating solution travelling with speed $v_{N}$, given by $\phi_{k}(t)=\phi^{(0)}\left(k-v_{N} t\right)$ and obeying the deterministic Eq. (14). However, the uniformly translating profile is suitable everywhere but few lattice sites at the tip of the front leading up to the f.o.l.s. The uniformly translating region is further subdivided into two parts — in the "linear region", the nonlinear term $\left[\phi^{(0)}\right]^{2}$ can be neglected. In the "nonlinear region", however, all the terms of Eq. (5) have to be taken into account.

Having taken this into consideration, the following scenario was proposed in Ref. [86, 87]: the lattice and finite particle effects lead to a halt-and-go dynamics at the f.o.l.s., while reasonably far behind, the average front "crosses over" to a uniformly translating (conditionally averaged) solution (see Fig. 9). In this formulation, the effect of stochasticity on the asymptotic front speed is coded in the probability distribution of the times required for the advancement of the f.o.l.s. The goal therein has been to develop a separate probabilistic theory for the particle hops to create the new f.o.l.s., and then to demonstrate by matching the front solution on the f.o.l.s. with the more standard one (of growth and uniform translation) reasonably far behind it in a mean-field type approximation at the "tip region" of the front, ${ }^{25}$ one obtains a self-consistent description of the stochastic and discreteness effects on front propagation. Although this formalism is not predictive, it allows one to deal with much smaller values of $N$ than it is required for the $\ln ^{-2} N$ asymptotics to be applicable. Moreover, it has the right asymptotics — it shows that as the value of $N$ is increased, one does approach to a uniformly translating front profile all the way to one lattice site behind the f.o.l.s., justifying the validity

$\overline{25}$ The tip region of the front spans a few lattice sites right behind the f.o.l.s. and also includes the f.o.l.s. (see Fig. 9). 
of the use of the conditionally averaged uniformly translating profile all over the front region, used in Refs. $[19,86,87,122,125,126]$ for the large- $N$ asymptotics (7). Below we provide few mathematical details of this procedure and present the key results, while the full details can be found in Ref. [86,87]. We note here that the formalism therein was developed for the reaction-diffusion system $\mathrm{X} \leftrightharpoons 2 \mathrm{X}$, but in principle it can be worked out for any other microscopic model of fluctuating "pulled" fronts.

The difficulty in prediction of $v_{N}$ in this formalism is the following. Microscopically, the selection process for the length of the time span between two consecutive f.o.l.s. creations is stochastic, and having followed the movements of the f.o.l.s. of one single front realization over a long time at large times, and thereafter having denoted the $j$ successive values of the duration of halts of the f.o.l.s. by $\Delta t_{1}, \Delta t_{2}, \ldots, \Delta t_{j}\left(\Delta t_{j^{\prime}} \geq 0 \forall j^{\prime}\right)$, one defines the front speed as the inverse of the long time average of this time span, i.e.,

$$
v_{N}=\lim _{j \rightarrow \infty} j\left[\sum_{j^{\prime}=1}^{j} \Delta t_{j^{\prime}}\right]^{-1} .
$$

Simultaneously, the amount of growth of particle numbers on and behind the f.o.l.s. itself depends on the time span between two consecutive f.o.l.s. creations (the longer the time span, the longer the amount of growth). As a consequence, on average, the selection mechanism for the length of the time span between two consecutive f.o.l.s. movements, which determines the asymptotic front speed, is nonlinear.

This inherent nonlinearity makes the prediction of the asymptotic front speed difficult. One might recall the difficulties associated with the prediction of pushed fronts due to nonlinear terms in this context, although the nature of the nonlinearities in these two cases is completely different. In the case of pushed fronts, the asymptotic front speed is determined by the mean-field dynamics of the fronts, and the nonlinearities originate from the nonlinear growth terms in the partial differential equations that describe the mean-field dynamics. On the other hand, for fronts consisting of discrete particles on a discrete lattice, the corresponding mean-field growth terms in the tip region of the front are linear. However, since the asymptotic front speed is determined from the probability distribution of the time span between two consecutive f.o.l.s. movements, on average, it is the relation between this probability distribution and the effect of the linear growth terms that the nonlinearities stem from (and makes it very difficult to theoretically deal with).

If we now denote the probability that a f.o.l.s. remains the f.o.l.s. for time $\Delta t$ by $\mathcal{P}(\Delta t)$, then the asymptotic front speed of Eq. (12) is given by ${ }^{26}$

\footnotetext{
${ }^{26}$ In this form, one starts the clock at zero as soon as a lattice site attains the status
} 


$$
v_{N}=\langle\Delta t\rangle^{-1}=\left[\int_{0}^{\infty} d(\Delta t) \Delta t \mathcal{P}(\Delta t)\right]^{-1}
$$

As outlined before, the theoretical expression of $\mathcal{P}(\Delta t)$ is obtained from the crossover of the mean-field type front solution at the tip region to the uniformly translating front solution behind. The front solution in the uniformly translating region (of Fig. 9) is given by $\phi^{(0)}\left(k-v_{N} \Delta t\right)$, such that

$$
\frac{\partial \phi_{k}^{(0)}}{\partial(\Delta t)}=D\left[\phi_{k+1}^{(0)}+\phi_{k-1}^{(0)}-2 \phi_{k}^{(0)}\right]+\phi_{k}^{(0)}-\left[\phi_{k}^{(0)}\right]^{2}
$$

where $D$ is the rate of diffusion of particles to their nearest neighbour lattice sites in the reaction-diffusion system $\mathrm{X} \leftrightharpoons 2 \mathrm{X}$. The uniformly translating region can be further subdivided into two parts, a "linear region" and a "nonlinear region", where the nonlinear $\left[\phi^{(0)}\right]^{2}$ term can and cannot be neglected respectively. On the other hand, in the mean-field type description of the tip region, the front solution is written as $\phi^{(0)}\left(k-v_{N} \Delta t\right)+\delta \phi_{k}(\Delta t)$. The quantities $\delta \phi_{k}(\Delta t)$ are expected to be strictly positive due to the halt-and-go motion of the f.o.l.s. - during the time the f.o.l.s. has halted at a given lattice site, the accumulation of particles flowing in from behind should result in an excess of "particle density buildup" over the uniformly translating solution $\phi^{(0)}\left(k-v_{N} \Delta t\right)$ at the tip region. Finally, in this formalism, the crossover point from the tip region to the uniformly translating region is located at the site, where $\delta \phi_{k}(\Delta t) / \phi^{(0)}\left(k-v_{N} \Delta t\right)$ remains negligibly small $\forall \Delta t$.
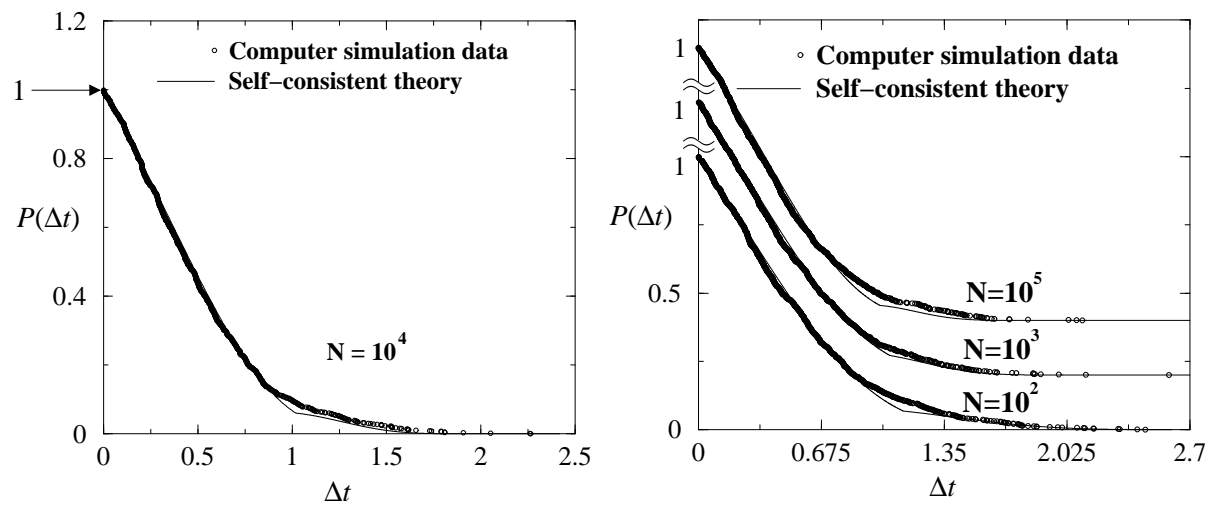

Fig. 10. The agreement between the self-consistent theoretical curve of $P(\Delta t)[86,87]$ and computer simulation results.

It is clear that to obtain the expression of $\mathcal{P}(\Delta t)$, one needs the expressions of $\phi^{(0)}\left(k-v_{N} \Delta t\right)$ and $\delta \phi_{k}(\Delta t)$. On the other hand, the expression of $v_{N}$ itself is needed to solve for $\phi^{(0)}\left(k-v_{N} \Delta t\right)$, and $v_{N}$ can be determined only from $\mathcal{P}(\Delta t)$

of the f.o.l.s. Thereafter, as soon as the next new f.o.l.s. is created, the clock reading is reset to zero. 
as Eq. (9) shows. This indicates that the only way to obtain the expression of $\mathcal{P}(\Delta t)$ is to solve a whole system of equations self-consistently [86,87]. We also note here that in this self-consistent formalism, there is an effective parameter.
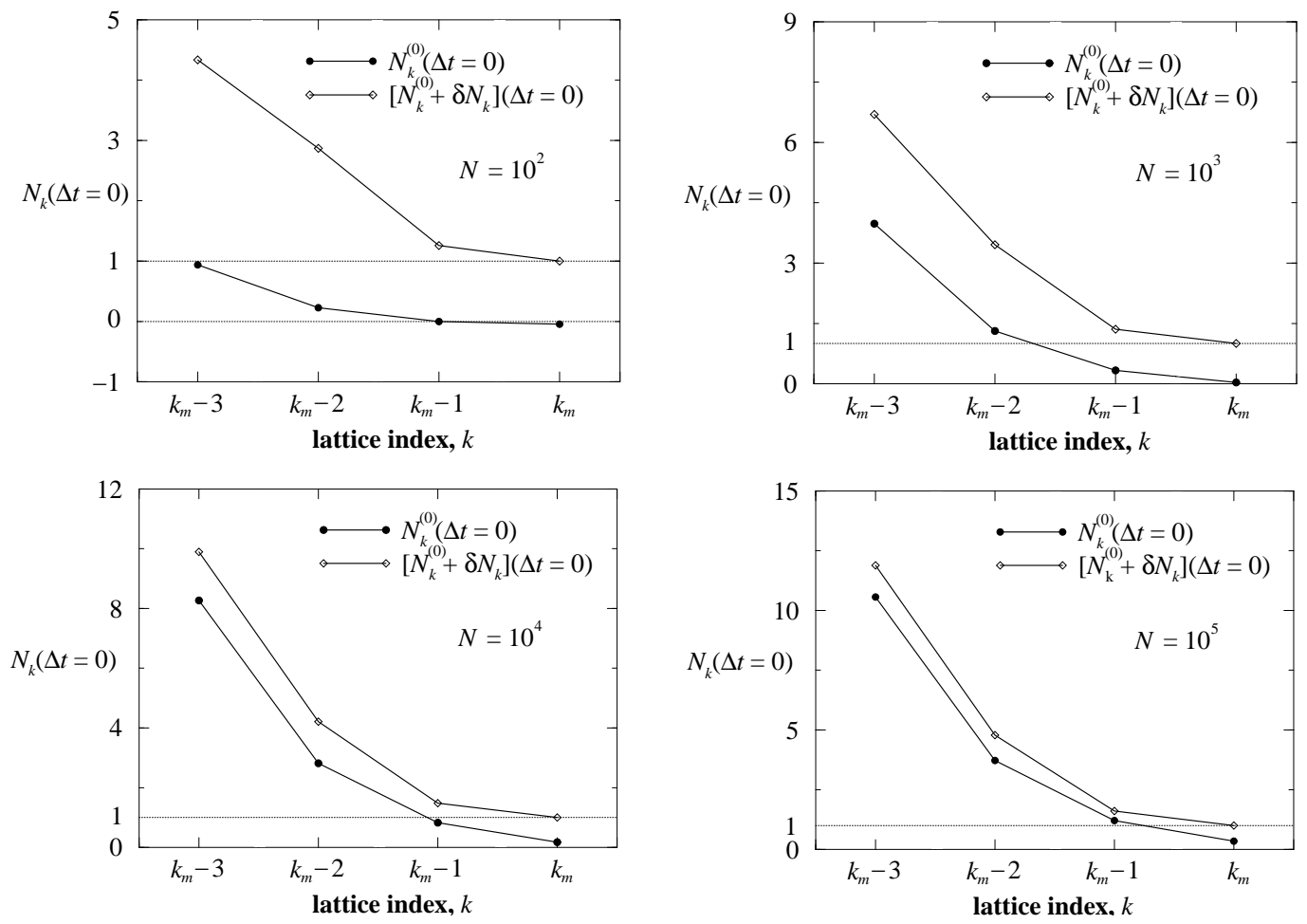

Fig. 11. Comparison between the $\delta N_{k}(\Delta t=0)=N \delta \phi(\Delta t=0)$ and the $N_{k}^{(0)}(\Delta t=0)=N \phi^{(0)}(k)$ values obtained from the mean-field type theory of $[86,87]$ for four foremost occupied lattice sites (the f.o.l.s. is indexed by $k_{m}$ ) and for $N=10^{2}$, $10^{3}, 10^{4}$ and $10^{5}$. Note that as $N$ increases, the $\delta N_{k}(\Delta t=0)$ values compared to the $N_{k}^{(0)}(\Delta t=0)$ profile become less and less important.

The corresponding self-consistent set of equations are highly nonlinear and complicated, but due to the presence of the effective parameter in this formalism, the procedure to obtain the $\mathcal{P}(\Delta t)$ in this formalism is not predictive. However, the fact that it generates a probability distribution that agrees so well with numerical simulations is indicative of the essential correctness of such a description of a fluctuating "pulled" front. The self-consistent theoretical curves of $P(\Delta t)=\int_{\Delta t}^{\infty} d t^{\prime} \mathcal{P}\left(t^{\prime}\right)$ for $D=1$ and $N=10^{4}, 10^{2}, 10^{3}$ and $10^{5}$ (in that order) are shown in Fig. 10. The corresponding numerical comparison of front speeds are shown in Table I. First, we notice that in the graphs of Fig. 10, the theoretical curves lie below the simulation histograms at $\Delta t \simeq 2 / v_{N}$ - this is an artifact of the matching that one has to carry out for the expressions of $P(\Delta t)$ below and above $\Delta t \simeq 2 / v_{N}$. This difference occurs due to certain fluctuation and correlation effects $[86,87]$. Secondly, the agreement between the simulation data and the self-consistent formalism is not very good 
for $N=10^{5}$ - at this value of $N$, the simulation gets very slow and one has to continuously remove particles from the stable phase of the front to gain program speed, which affects the $P(\Delta t)$ histograms significantly for large $\Delta t$ values.

\begin{tabular}{||c|c|c|c||}
\hline \hline$N$ & $v_{N}$ (simulation) & $v_{N}$ (theoretical) & $v_{N}[$ Eq. (7)] \\
\hline $10^{2}$ & 1.778 & 1.808 & 1.465 \\
\hline $10^{3}$ & 1.901 & 1.899 & 1.803 \\
\hline $10^{4}$ & 1.964 & 1.988 & 1.925 \\
\hline $10^{5}$ & 2.001 & 2.057 & 1.976 \\
\hline \hline
\end{tabular}

Table II: Comparison of $v_{N}$ values: simulation, self-consistent formalism $[86,87]$ [denoted by $v_{N}$ (theoretical)], and that of the large $N$ asymptotics (7).

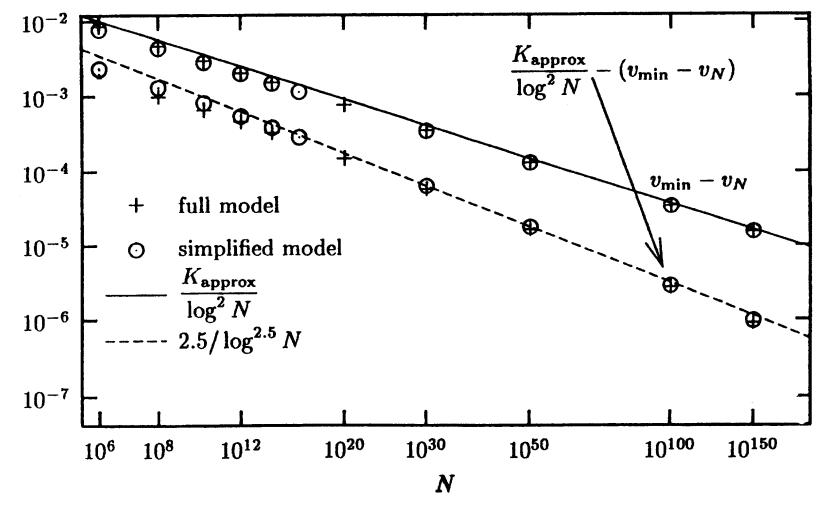

Fig. 12. Comparison between the actual stochastic model and the corresponding "simplified model" relating to directed polymers in random media [21]. For the large $N$ asymptotics of $v_{N}$ and the leading order $N$-dependent behaviour of the subdominant corrections to it, note the agreement between the results of the two models. Here, $v_{\min }$ is $v^{*}$, and $K_{\text {approx }}$ is the coefficient of $1 / \ln ^{2} N$ in Eq. (7). The figure has been modified from the original for greater clarity.

In addition to good agreements between the self-consistent formalism and simulations for the $P(\Delta t)$ curves in Fig. 10, a significant observation of the formalism is the following: as the value of $N$ is increased in the self-consistent formalism, it is found that the $\delta \phi_{k} / \phi_{k}^{(0)}$ values at the tip region of the front gradually reduces $[86,87]$ (see Fig. 11). The stochastic halt-and-go character of the movement of the f.o.l.s., which is usually occupied by $\mathcal{O}(1)$ number of particles, however, continues to remain valid for any value of $N$. This implies that for very large $N$, one approaches the picture of a fluctuating "pulled" front, where a uniformly translating mean-field description (5) holds all the way up to one lattice site behind the f.o.l.s., while only the dynamics of the f.o.l.s. remains a stochastic halt-and-go process. Such a scenario was numerically in- 
vestigated in Ref. [21] by means of constructing a "simplified model", in which stochasticity was introduced only on the f.o.l.s., while the rest of the front was described by a deterministic equation. A comparison of the simulation data of the actual stochastic model (the "full model") and the simplified model from Ref. [21] is shown in Fig. 12. The agreement between the two cases - not only at the level of large $N$ asymptotics (7), but also the leading order $N$-dependent behaviour of the subdominant corrections (c.f. the last paragraph of Sec. 2.2) - suggests that the "simplified model" is a very good representation of the true stochastic model at asymptotically large $N$ values. ${ }^{27}$

\subsection{The weakly pushed nature of fluctuating "pulled" fronts}

\subsection{1 (In)dependence of the front shape and speed on the initial configuration}

One common aspect of propagating fronts in the microscopic models of fluctuating fronts is that at a given value of $\Delta t$, the more particles there are on the f.o.l.s., the more likely it is for the f.o.l.s. to move forward. This indicates that if there are more particles put on the f.o.l.s. in these models at short values of $\Delta t$, the faster the f.o.l.s. moves.

In reaction-diffusion models, the number of particles on the f.o.l.s. can increase by means of two processes: flow of particles from the other lattice sites in the tip region behind the f.o.l.s., and growth of particles on the f.o.l.s. by themselves without having any input of particles from other lattice sites. In the so-called clock model [122-126] or the discrete particle and lattice model related to directed polymers in random media [19-21,24,27,28], the number of particles on the f.o.l.s. can increase only by means of flow of particles from the other lattice sites behind. For the two latter models, when a conditionally averaged steady shape of the front emerges at long times, a complicated balance develops between $\langle\Delta t\rangle=v_{N}^{-1}$ (i.e., on average for how long the f.o.l.s. halts at a given lattice site) and the average flow of particles on to the f.o.l.s. from behind, and the balance is established at $v_{N}<v^{*}$. Such a balance also develops for the reaction-diffusion models, but it is more complicated in nature - the particles that flow on to the f.o.l.s. can start their own growth, thereby further contributing to the increasing number of particles on the f.o.l.s.

The observation above demonstrates the pushed ${ }^{28}$ nature of these fronts, as

\footnotetext{
$\overline{{ }^{27} \text { Such }}$ a statement goes beyond the consideration of simply the front speed, as the front diffusion coefficients for the actual stochastic model and the "simplified model" show no difference for asymptotically large $N$ values. We will address the issue of front diffusion later in Sec. 2.5.

28 The above argument is not quantitative, but we have seen earlier that the asymptotic front speed $v_{N}$ differs from $v^{*}$ by only a small amount for large values of $N$.
} 
their speed of propagation depends on the front solution far behind the f.o.l.s. The front solution far behind the f.o.l.s. contributes to the movements of the f.o.l.s. in the following way: a uniformly translating solution exists behind the tip region (see Fig. 9), and for the time the f.o.l.s. halts at a given lattice site, the uniformly translating region of the front brings in particles into the tip region - ultimately that contributes to how fast the f.o.l.s. moves forward. The supply of particles from behind is therefore a very crucial ingredient for the front (along with the f.o.l.s.) to propagate at a speed $v_{N}$, indicating that if the supply of particles on to the f.o.l.s. from behind were cut off, the speed of the f.o.l.s. would reduce drastically (this is already illustrated by the discussion of the first two paragraphs of Sec. 2.2, where the "inertia" of the f.o.l.s. against its forward movement due to the discreteness of particles and the lattice effects are mimicked by a cutoff). It also indicates that if the number of particles on the f.o.l.s. are increased artificially in these models at small values of $\Delta t$, the front should propagate faster. Such a model was investigated in Ref. [88] in a reaction-diffusion system with enhanced growth rate of particles in the tip region, resulting in a front that propagates with a speed significantly higher than $v^{*}{ }^{29}$

On the basis of the above two paragraphs, the independence of the front speed and the conditionally averaged front shape on the initial configuration can be argued in the following manner, although no formal proof exists in the literature. ${ }^{30}$ When a discrete particle and lattice system of a fluctuating "pulled" front has not reached its conditionally averaged steady shape, the balance between the average value of $\Delta t$ and the average flow of particles on to the f.o.l.s. from behind does not hold. For example, consider an initial microscopic configuration, when the front shape at the leading edge resembles that of the functional form $\exp [-\lambda k]$, with $\lambda=\lambda_{>}>\lambda^{*}$. With such a configuration, "linear region" initially propagates with a speed $v\left(\lambda_{>}\right)>v^{*}$, and bring in more particles on to the tip region (and eventually on the f.o.l.s.) than what it does

This is why we call them weakly pushed. We will see later that such a nomenclature is also supported by the spectrum of $\mathcal{L}_{v_{N}}$.

${ }^{29}$ As readers can find out, Ref. [88] studies a continuum space Fisher-Kolmogorov equation type model, where the cutoff in the growth function at $\phi=\varepsilon$ is supplemented by an enhancement in growth between $\varepsilon<\phi \leq \varepsilon / r$ for $r<1$. Of course, as $\varepsilon \rightarrow 0$, the evolution equation of the front reaches its pulled front limit (FisherKolmogorov equation). The analysis however shows that first taking the $\varepsilon \rightarrow 0$ limit to obtain the Fisher-Kolmogorov equation and then obtaining the front speed $\left(v^{*}\right)$ is not right - one has to first calculate the front speed as a function of $\varepsilon$ and $r$ and only then take the limit $\varepsilon \rightarrow 0$. Interestingly enough, it turns out that when $r \leq r_{c}=0.283833 \ldots$, the front speed converges to $v^{*}$, otherwise the front speed can be significantly higher than $v^{*}$.

${ }^{30}$ As we have seen in Sec. 2.3, the balance between how long the f.o.l.s. halts at a given lattice site and the average flow of particles on to the f.o.l.s. from behind is very complicated. In view of this, it is unlikely that a formal proof can be obtained. 
at the steady state, speeding up the f.o.l.s. Subsequently, the "inertia" of the f.o.l.s. also gets communicated to the uniformly translating front solution behind, which then starts to slow down. A combination of these two phenomena makes the leading edge less and less steep, until the front finally settles to its steady shape (an illustration of it can be found in Fig. 1 of Ref. [16]). Contrast this situation with an initial microscopic configuration that resembles the functional form $\exp [-\lambda k]$, with $\lambda=\lambda_{<}<\lambda^{*}$, such that $v\left(\lambda_{<}\right)=v\left(\lambda_{>}\right)$. In this case, the "linear region" initially propagates with the same speed as before, but since it is less steep than $\exp \left[-\lambda_{>} k\right]$, it does not bring in as many particles on to the f.o.l.s. as it does for $\lambda=\lambda_{>}$, and as a result, the f.o.l.s. does not speed up as much as it did before. By means of a simple comparison of couple of diagrams contrasting these two cases, one can easily convince oneself that the front profile at the leading edge in this case continues to becomes steeper and steeper. Although there does not exist any specific simulation data showing that for arbitrary initial configuration, the front ultimately settles down to the same conditionally averaged front shape (and speed), the arguments above strongly suggest that it is indeed the case.

\subsubsection{The Large $N$ Asymptotic Spectrum of $\mathcal{L}_{v_{N}}$}

To obtain the large $N$ asymptotic spectrum of $\mathcal{L}_{v_{N}}$, we follow the procedure developed in Refs. [56,89], which in turn use the standard route of transforming the linear eigenvalue problem into a Schrödinger eigenvalue problem [12,22, $23,38,92]$. To represent a discrete particle and lattice system of a fluctuating "pulled" front" as $N \rightarrow \infty$, the idea is to consider the cutoff picture (8) in continuum space, developed by Brunet and Derrida.

In the comoving co-ordinate $\xi=x-v_{N} t$, Eq. (8) in the steady state reads

$$
-v_{N} \frac{d \phi^{(0)}(\xi)}{d \xi}=\frac{d^{2} \phi^{(0)}(\xi)}{d \xi^{2}}+f\left[\phi^{(0)}(\xi)\right],
$$

with $f\left[\phi^{(0)}(\xi)\right]=\left\{\phi^{(0)}(\xi)-\left[\phi^{(0)}(\xi)\right]^{n}\right\} \Theta\left[\phi^{(0)}(\xi)-1 / N\right]$. Now consider a function $\phi(x, t)$ infinitesimally different from $\phi^{(0)}(\xi) \equiv \phi^{(0)}\left(x-v_{N} t\right)$ in the comoving frame, i.e., $\phi(x, t)=\phi^{(0)}\left(x-v_{N} t\right)+\eta(\xi, t)$. Upon linearizing the dynamical equation in the comoving frame, one finds that the function $\eta(x, t) \equiv \eta(\xi, t)$ obeys the following equation:

$$
\frac{\partial \eta}{\partial t}=\mathcal{L}_{v_{N}} \eta=\left[v_{N} \frac{\partial}{\partial \xi}+\frac{\partial^{2}}{\partial \xi^{2}}+\left.\frac{\delta f(\phi)}{\delta \phi}\right|_{\phi=\phi^{(0)}}\right] \eta
$$

In this formalism, the substitution $\eta(\xi, t)=e^{-E t} e^{-v_{N} \xi / 2} \psi_{E}(\xi)$ converts Eq. (16) to the following one-dimensional Schrödinger equation for a particle in a 
potential (with $\hbar^{2} / 2 m=1$ ) $[12,22,23,38,92]$ :

$$
\left[-\frac{d^{2}}{d \xi^{2}}+\frac{v_{N}^{2}}{4}-\left.\frac{\delta f(\phi)}{\delta \phi}\right|_{\phi=\phi^{(0)}}\right] \psi_{E}(\xi)=E \psi_{E}(\xi)
$$

In Eq. (17), the quantity $V(\xi)=\left[\frac{v_{N}^{2}}{4}-\left.\frac{\delta f(\phi)}{\delta \phi}\right|_{\phi=\phi^{(0)}}\right]$ plays the role of the potential. Having denoted the the coordinate of the point where $\phi^{(0)}(\xi)=1 / N$ by $\xi_{0}$ as before, we have

$$
V(\xi)=\left[\frac{v_{N}^{2}}{4}-1+n\left[\phi^{(0)}\right]^{n-1}\right] \Theta\left(\xi_{0}-\xi\right)-\frac{1}{v_{N}} \delta\left(\xi-\xi_{0}\right)+\frac{v_{N}^{2}}{4} \Theta\left(\xi-\xi_{0}\right) .
$$

The form of the potential $V(\xi)$ is sketched in the left figure of Fig. 13. Notice that $\phi^{(0)}(\xi)$ is a monotonically increasing function from $1 / N$ at $\xi_{0}$ towards the left, asymptotically reaching the value 1 as $\xi \rightarrow-\infty$. As a result, for $\xi<\xi_{0}, V(\xi)$ also increases monotonically towards the left, from $v_{N}^{2} / 4-1+$ $n N^{1-n} \simeq-\pi^{2} / \ln ^{2} N$ at $\xi=\xi_{0-}$, to $\left(n-\pi^{2} / \ln ^{2} N\right) \approx n$ as $\xi \rightarrow-\infty$. At $\xi_{0}$, there is an attractive $\delta$-function potential of strength $1 / v_{N} \approx 1 / 2$ and a finite step of height 1 . The crucial feature for the stability analysis below is the fact that $V(\xi)$ stays remarkably flat at a value $-\pi^{2} / \ln ^{2} N$ over a distance $\left(\xi_{0}-\xi_{1}\right) \simeq \ln N$, and then on the left of $\xi_{1}$, it increases to the value $\approx n$, over a distance of order unity. This is a consequence of the nature of the solution (10).
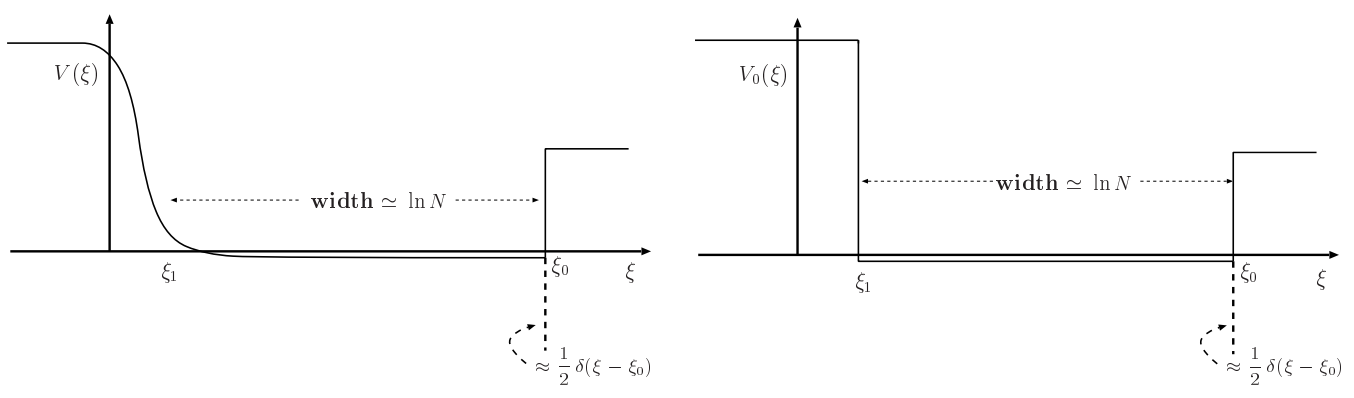

Fig. 13. Left: The potential $V(\xi)$ in the Schrödinger operator obtained in the stability analysis. Right: The approximate potential $V_{0}(\xi)$ that can be used for calculating the low-lying modes for large widths of the bottom well, i.e., for large $N$.

The question of stability of the front solution can be answered by studying the spectrum of the temporal eigenvalues. If there are negative eigenvalues of the above Schrödinger equation, then according to Eq. (16) $\eta(\xi, t)$ grows in time in the comoving frame, i.e., the front solution $\phi^{(0)}(\xi)$ is unstable. On the other hand, if there are no negative eigenvalues, then the asymptotic front shape is stable, and the spectrum of the eigenvalues then determines the nature of the 
relaxation of $\phi(x, t)$ to the solution $\phi^{(0)}(\xi)$. The full spectrum in general depends on the boundary conditions imposed on the eigenfunctions $\psi_{E}$. Here we consider only localized perturbations, for which we need to have $\eta(\xi, t) \rightarrow 0$ as $\xi \rightarrow \pm \infty$. Because of the exponential factor in Eq. (16), any eigenfunction $\psi_{E}$ which vanishes as $\xi \rightarrow \infty$ is consistent with vanishing $\eta$ towards the right ${ }^{31}$. However, for $\xi \rightarrow-\infty$, the eigenfunctions $\psi_{E}$ need to vanish exponentially fast with a sufficiently large exponent, so that when it is combined with the exponentially diverging term $e^{-v_{N} / 2}$, they are still consistent with the requirement that $\eta$ vanishes for $\xi \rightarrow-\infty$. For the lowest "energy" eigenvalues investigated below, we demonstrate that these requirements are obeyed.

From the form in the potential, it is clear that the lowest "energy" eigenmodes, i.e., the slowest relaxation eigenmodes, are the ones that are confined to the bottom of the potential. We notice that among these modes, invariably there is a zero mode of the stability operator that is associated with the uniformly translating front solution of a dynamical equation like Eq. (8): since $\phi^{(0)}(\xi)$ and $\phi^{(0)}(\xi+a)$ are solutions of Eq. (14) for any arbitrary $a$, we find by expanding to first order in a that $\psi_{0}(\xi)=e^{v_{N} \xi / 2} \frac{d \phi^{(0)}}{d \xi}$ is a solution of Eq. (17) with eigenvalue $E=0 .{ }^{32}$ From the result (19) for the asymptotic front solution, we then immediately get, to dominant order,

$$
\psi_{0} \sim \sin \left[\pi \lambda^{*}\left(\xi-\xi_{1}\right) / \ln N\right] \quad 0 \simeq \xi_{1} \lesssim \xi \leq \xi_{0}
$$

Furthermore, since $\phi^{(0)}(\xi)$ is a monotonically decreasing function of $\xi$, the solution $\psi_{0}(\xi)=e^{v_{N} \xi / 2} \frac{d \phi^{(0)}}{d \xi}$ is nodeless. Since we know from elementary quantum mechanics that the nodeless eigenfunction has the lowest eigenvalue, this implies that all the other eigenvalues of Eq. (17) are positive, i.e., the solution $\phi^{(0)}(\xi)$ is stable.

The spectrum of eigenvalues of Eq. (17) for $E>0$ determines the decay property of localized perturbations $\eta(\xi, t)$ in time. We notice that for $E>v_{N}^{2} / 4 \approx 1$ (the value of the potential on the far right), the spectrum of eigenvalues are continuous. However, we are particularly interested in the smallest eigenvalues $E_{m}>0$ for small $m$, since these are the eigenmodes that decay the slowest in time. These are the eigenvalues associated with bound states in the potential well. As $N \rightarrow \infty$, the bottom well of the potential becomes very wide: its

\footnotetext{
$\overline{31}$ The fact that eigenfunctions $\psi_{E}$ which diverge as $\xi \rightarrow \infty$ are allowed, means that there are admissible eigenfunctions which are not in the Hilbert space of the Schrödinger operator. See e.g., Ref. [38] for further discussion of this point.

${ }^{32}$ We will later see in Sec. 3.1.2 that $\frac{d \phi^{(0)}}{d \xi}$ is also known as the so-called Goldstone mode $[94,109,110]$.
} 
width diverges as $\ln N$. As we know from elementary quantum mechanics, the lowest "energy" eigenfunctions then become essentially sine or cosine waves in the potential well with small wave numbers $k$ and correspondingly small "energy" eigenvalues. Based on the fact that the potential $V(\xi)$ on the left rises over length scales of order unity, we now make an approximation. In the limit that the bottom well is very wide and the $k$ values of the bound state eigenmodes very small, it becomes an increasingly good and an asymptotically correct approximation to view the left wall of the well as a steep step, sketched in the right figure of Fig. 13 - one can therefore approximate the potential by

$$
\begin{aligned}
& V_{0}(\xi)=n[1-\Theta(\xi)]-\frac{\pi^{2}}{\ln ^{2} N} \Theta(\xi) {\left[1-\Theta\left(\xi-\xi_{0}\right)\right] } \\
&-\frac{1}{2} \delta\left(\xi-\xi_{0}\right)+\Theta\left(\xi-\xi_{0}\right) .
\end{aligned}
$$

On the right hand side, there is an attractive delta-function potential at the point where the potential shows a step to a value close to 1 . It is easy to check that the prefactor of the delta-function of $1 / 2$ is not strong enough to give rise to bound states with $E<0$, and as a result, for very large values of $N$, the low-lying eigenmodes approach sine waves with nodes at the position of the walls of the potential, ${ }^{33} \psi_{m} \simeq \sin \left[q_{m}\left(\xi-\xi_{1}\right)\right]$. The condition that these solutions have nodes at the right edge of the well then yields

$$
q_{m} \simeq \frac{(m+1) \pi}{\xi_{0}-\xi_{1}} \simeq \frac{(m+1) \pi}{\ln N}
$$

implying that the corresponding eigenvalues are given by ${ }^{34}$

$$
E_{m} \simeq \frac{\left[(m+1)^{2}-1\right] \pi^{2}}{\ln ^{2} N} \quad m=0,1,2, \ldots
$$

Here, the first term between square brackets comes from the "kinetic energy" term $q^{2}$, while the second term originates from the value of the potential at

$\overline{33}$ More explicitly, if we write the solution within the well as $A \sin \left[q\left(\xi-\xi_{1}\right)+B\right]$, and for $\xi>\xi_{0}$ as $A_{+} e^{-\left(\xi-\xi_{0}\right)}$ (which is correct to lowest order in $\epsilon$ ), then we get from the boundary conditions at $\xi_{0}: 2 q \operatorname{cotg}\left[q\left(\xi_{0}-\xi_{1}\right)+B\right]=-1$; likewise, at the left boundary, of the well, we get $q \operatorname{cotg} B=$ const., where the constant is determined by the size of potential step. For $\xi_{0}-\xi_{1} \gg 1$, the small- $q$ solutions are those with $B \rightarrow 0$.

${ }^{34}$ Notice that for the eigenvalues in Eq. (22), the corresponding eigenmodes $\psi_{E}(\xi)$ decay as $\exp [-\sqrt{n}|\xi|]$ for $\xi \rightarrow-\infty$ and as $\exp \left[-v_{N} \xi / 2\right]$ for $\xi \rightarrow \infty$, which make $e^{v_{N} \xi / 2} \psi_{E}(\xi)$ to go to zero for $\xi \rightarrow \pm \infty$, satisfying the boundary conditions discussed previously. 
the bottom. Note that for $m=0$, the eigenmode $\sin q_{0}$ with eigenvalue $E_{0}$ is indeed the same as the zero eigenmode of Eq. (19) with $q_{0}=\pi \lambda^{*} / \ln N$, which we calculated from the shape of the front solution $\phi^{(0)}$ in the leading edge. Besides verifying the consistency of our approach, this also confirms that there are no corrections to Eq. (22) for $m=0$ : for $m=0$, it will yield an eigenvalue zero to all orders in $N$. Therefore, the smallest nonzero eigenvalue, which governs the relaxation of the front velocity and profile to the asymptotic ones is $E_{1}$ with relaxation time $\tau_{1}$ given by

$$
\tau_{1}^{-1}=E_{1} \simeq \frac{3 \pi^{2}}{\ln ^{2} N}
$$

The time convergence of the asymptotic front speed for the reaction-diffusion

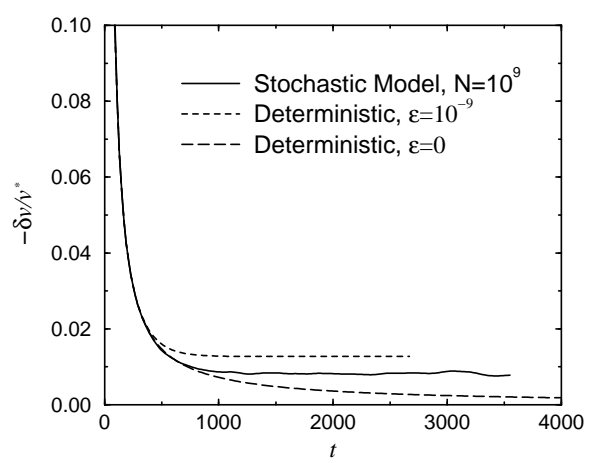

Fig. 14. The time convergence of the asymptotic front speeds: for Eq. (8) [short dashed line], and for the stochastic reaction-diffusion system $\mathrm{X}+\mathrm{Y} \rightarrow 2 \mathrm{X}$ (solid line) [56]. They follow corresponding curve for the deterministic equation (1) with $n=2$ (long dashed line) for a while, and then they suddenly break off to settle down to their asymptotic values. As the long dashed line represents the power law convergence $t^{-1}$ for pulled fronts, such a behaviour for the solid and the short dashed curve is expected for a very slow exponential decay of Eq. (23). Note that the notations have been changed from the original to suit the present article. Here $\delta v=v^{*}-v$.

system $\mathrm{X}+\mathrm{Y} \rightarrow 2 \mathrm{X}$ is shown in the simulation data from Ref. [56] (Fig. 14), in support of Eq. (23). As can be seen therein, as a function of time, the front speed for Eq. (8) [short dashed line] and for the stochastic reaction-diffusion system $\mathrm{X}+\mathrm{Y} \rightarrow 2 \mathrm{X}$ (solid line) follow corresponding curve for the deterministic equation (1) with $n=2$ (long dashed line) for a while, and then they suddenly break off to settle down to their asymptotic values. As the long dashed line represents the power law convergence $t^{-1}$ for pulled fronts, such a behaviour for the solid and the short dashed curve is expected for a very slow exponential decay of Eq. (23).

We have noted earlier in the introduction that for a pushed front, the lowest lying eigenspectrum is gapped. For discrete particle and lattice systems of a fluctuating "pulled" front with $N \rightarrow \infty$, according to the above analysis, the 
spectrum is also gapped, but the gap is very small, as it is $\propto \ln ^{-2} N$. This is another confirmation of these fronts being weakly pushed. Moreover, Eq. (22) also confirms that as $N \rightarrow \infty$, the gap between the spectral lines decreases as $\ln ^{-2} N$, which is consistent with the fact that for a pulled front $N=\infty$ and the spectrum becomes gapless (see footnote 12 in this context).

In connection to the gapless spectrum in the limit $N \rightarrow \infty$, a curious observation was made in Ref. [56]. Put simply, the observation is the following: in the time convergence of the front speed to its asymptotic value $v^{*}$, the leading edge increases in length $\sim \sqrt{t}$ leading to the uniform $t^{-1}$ convergence (4). For a discrete particle and lattice system of fluctuating "pulled" fronts with $N \rightarrow \infty$, the length of the leading edge in Eq. (10) increases as $\ln N$, which ultimately leads to the $1 / \ln ^{2} N$ deviation of the asymptotic front speed $v_{N}$ from $v^{*}$ (although in this similarity the prefactors do not match correctly). Based on these observation, Kessler and co-workers suggested [56] that the initial conditions, which are responsible for the front speed selection of a pulled front, act as an effective time-dependent cutoff beyond which the steady state equation no longer holds, and the time-dependent cutoff goes to zero with increasing time $^{35}$. To what extent there is a deep physics underlying this observation, however, is not clear.

\subsection{The Large $N$ Asymptotic Scaling of Front Diffusion Coefficient $D_{f}$ for Fluctuating "Pulled" Fronts}

So far, we have only dealt with (and observed) how the discreteness of particles and the lattice sites affect the asymptotic front speed of fluctuating "pulled" fronts. With the idea behind random wandering of fronts has been introduced already in Sec. 1.2, we now address the issue of their diffusive wandering.

It turns out that the theoretical concept of random wandering of fronts is actually an extremely tricky issue, the full flavour of which will only be clear in Secs. 3 and 4.2. However, operationally, i.e., in a computer simulation, the front diffusion for a front consisting of discrete particles on a lattice is rather straightforward to measure. Consider for example the fluctuating "pulled" front in the so-called clock model [122,124-126]. This model, which arose in the calculation of the largest Lyapunov exponent for a gas of hard spheres [122, 124-126], one considers a set of $N$ clocks with integral clock readings $k=0,1,2, \ldots$. The number of clocks with a certain reading $k$ at time $t$ is $n_{k}(t)$. Any two clocks can "collide" continuously in time; the post-collisional readings of a pair of mutually "colliding" clocks with pre-collisional readings $k_{i}$ and $k_{j}$ are both updated to $\max \left(k_{i}, k_{j}\right)+1$. In terms of $\phi_{k}(t)=\sum_{k^{\prime}=k}^{\infty} n_{k^{\prime}}(t) / N$

\footnotetext{
${ }^{35}$ A similar observation was also made therein about pushed fronts with cutoffs.
} 
(with time $t$ redefined to have a unit mean single clock collision frequency), the relevant asymptotic solution corresponding to an initial state in which all clock readings are finite is a front propagating into the state $\phi_{k}=0$ for large $k$. In the mean-field limit, the dynamics is that of a pulled front propagating with speed $v^{*}=4.31107 \ldots[122,124-126]$.

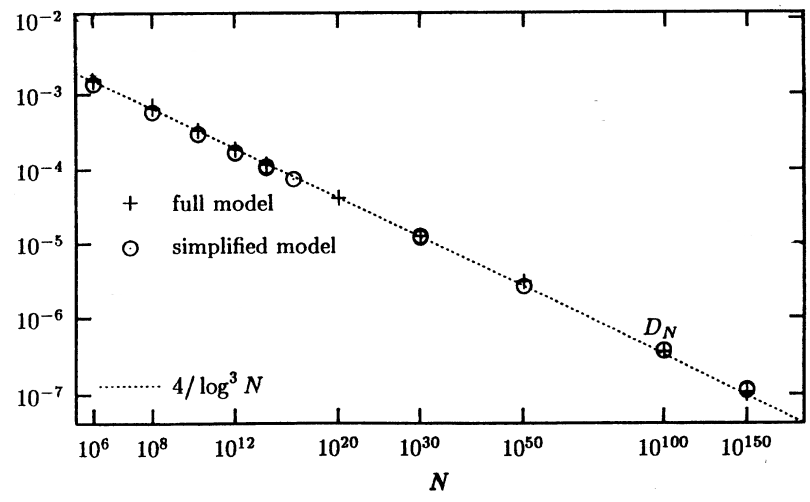

Fig. 15. Simulation data from Ref. [21] for the front diffusion coefficient, for both the microscopic stochastic model relating to directed polymers in random media ("full" model) and the "simplified" model. In the above figure, $D_{N}$ should be read as $D_{f}$. The figure has been modified from the original for greater clarity.

A straightforward way to measure the front speed and the front diffusion coefficient in a computer simulations of the clock model is by tracking the position of the centre of mass of the clock distribution in individual realizations as a function of time. With the centre of mass location for the clock distribution in an actual realization $\mathrm{r}$ at time $t$ given by

$$
S_{\mathrm{r}}(t)=N^{-1} \sum_{k} k n_{k}(t) \equiv \sum_{k} \phi_{k}(t)
$$

one has $v_{N}=\left\langle S_{\mathrm{r}}(t)\right\rangle$, and $D_{f}=\lim _{T \rightarrow \infty} d\left\langle\left[\int_{t}^{t+T} d t \dot{S}_{\mathrm{r}}(t)-v_{N} T\right]^{2}\right\rangle / d T$ [the angular brackets denote first an average over all possible evolution sequence for a given initial realization (at time $t$ ), and then a further averaging over the ensemble of initial realizations]. This was indeed the same method used by Brunet and Derrida to obtain the front speed and front diffusion coefficient [21] for a model which is slightly different from the clock model. The superb cleverness in their measurement of the front diffusion lies in the simulation algorithm they used - this algorithm allowed them to take $N$ as high as $10^{150}$, and they demonstrated that the $N \rightarrow \infty$ asymptotic scaling of front diffusion coefficient $D_{f}$ behaves as $1 / \ln ^{3} N$ [21]. Moreover, a "simplified model", where the fluctuations are randomly generated only on the instantaneous foremost occupied lattice site (i.f.o.l.s.), ${ }^{36}$ was found to exhibit the same $1 / \ln ^{3} N$

\footnotetext{
36 The i.f.o.l.s. is simply the location of the lattice site on the right of which all
} 
asymptotic scaling of $D_{f}$ as the full stochastic model [21]. However, we note that in the absence of any studies on the diffusion coefficient of fluctuating "pulled" fronts on other microscopic models, to what extent this scaling is independent of the model is not known.

For the clock model, we will provide a argument of the $1 / \ln ^{3} N$ asymptotic scaling of $D_{f}$ in Sec. 4.2, while presently we simply obtain the formal GreenKubo expression for $D_{f}$. Needless to say, dividing the derivation in this manner is complicated for the readers. Nevertheless, the reason behind postponing the derivation of the $1 / \ln ^{3} N$ asymptotic scaling of $D_{f}$ till Sec. 4.2 .2 is the following: it will require certain inputs and comparisons with the stochastic differential equation used in the Langevin-type field-theoretical approaches of fluctuating fronts, which we will discuss in Secs. 3 and 4.

The central theme to obtain $D_{f}$ is that there is stochasticity in the front evolution at two levels: first, in any snapshot of the clock model realization $\mathrm{r}$ at time $t$, its shape $\left\{n_{k}(t)\right\}_{\mathrm{r}}$ fluctuates around $\left\{n_{k}^{(0)}(t)\right\} \equiv\left\{n^{(0)}(\xi)\right\}$, where $n^{(0)}(\xi)=\phi^{(0)}(\xi)-\phi^{(0)}(\xi-1)$. In the subsequent front evolution, in an average or mean-fieldish sense, the clocks are chosen from a "distorted distribution" $\left\{n_{k}(t)\right\}_{\mathrm{r}}$ rather than from $\left\{n_{k}^{(0)}(t)\right\}$, and this introduces fluctuations in the front speed $\delta v_{\mathrm{r}}(t)$ [measured by tracking the centre of mass of the clocks]. Let us denote, by $\delta v_{\mathrm{r}, \mathrm{mf}}(t)$, this average fluctuations in the instantaneous front speed around $v_{N}$ at time $t$ due to the shape fluctuation of the front at time $t$. From the actual clocks that constitute the front, at time $t$, we have

$$
v_{N}+\delta v_{\mathrm{r}, \mathrm{mf}}(t)=\sum_{i, j}\left[2-\delta_{k_{i}, k_{j}}\right]\left(\left|k_{i}-k_{j}\right|+2\right)\left(n_{k_{i}}\left[n_{k_{j}}-\delta_{k_{i}, k_{j}}\right]\right) / 2 N^{2} .
$$

Equation (25) is obtained by applying a probabilistic argument in the microscopic dynamics of the clock model - in a collision between two clocks with readings $k_{i}$ and $k_{j}$, the mean clock reading increases by an amount $\left(\left|k_{i}-k_{j}\right|+2\right) / N$, the probability of having such a collision is $\left[2-\delta_{k_{i}, k_{j}}\right]\left[n_{k_{i}}\left(n_{k_{j}}-\right.\right.$ $\left.\left.\delta_{k_{i}, k_{j}}\right)\right] /[N(N-1)]$, and finally in a unit time, on average, $(N-1) / 2$ collisions occur. The $\delta_{k_{i}, k_{j}}$ 's arise due to the indistinguishability of the clocks with the same readings. When we write $\left\{n_{k}(t)\right\}_{\mathrm{r}}=n_{k}^{(0)}(t)+\left\{\delta n_{k}(t)\right\}_{\mathrm{r}}$, the expression of $v_{N}$ is obtained from Eq. (25) by replacing $\left\{n_{k}(t)\right\}_{\mathrm{r}}$ by $\left\{n_{k}^{(0)}(t)\right\}$ in the first step of Eq. (25), implying that at the leading order

$$
\delta v_{\mathrm{r}, \mathrm{mf}}(t)=\sum_{i, j}\left[2-\delta_{k_{i}, k_{j}}\right] \frac{\left|k_{i}-k_{j}\right|+2}{N} \frac{n_{k_{i}}^{(0)}\left[n_{k_{j}}^{(0)}-\delta_{k_{i}, k_{j}}\right]}{2 N} \times
$$

lattice sites are instantaneously empty. It is different from the f.o.l.s. of Sec. 2.3. 


$$
\times\left[\frac{\delta n_{k_{i}}}{n_{k_{i}}^{(0)}}+\frac{\delta n_{k_{j}}}{n_{k_{j}}^{(0)}-\delta_{k_{i}, k_{j}}}\right] .
$$

The idea behind writing $\delta v_{\mathrm{r}, \mathrm{mf}}(t)$ in this manner is to emphasize the fact that despite several factors of $N$ involved in the expression of $v_{N}$, its magnitude is of $\mathcal{O}(1)$; and therefore, the order of magnitude estimate of $\delta v_{\mathrm{r}, \mathrm{mf}}(t)$ must behave as the ratio of $\delta n_{k}(t)$ and $n_{k}^{(0)}(t)$ in realization $\mathrm{r}$.

Secondly, beyond (the mean-fieldish) $\delta v_{\mathrm{r}, \mathrm{mf}}(t)$, there is another source of the instantaneous front speed fluctuation that depends on what is the precise sequence of random numbers that updates the clock distribution in an infinitesimally short time window after $t$, i.e., between times $t$ and $t+\varepsilon$ for an infinitesimally small $\varepsilon$. Let us denote the front speed fluctuation due to the stochasticity in the front evolution at time $t$ by $\delta v_{\mathrm{r}, \mathrm{s}}\left(t_{+}\right)$[this depends on the updating sequence of random numbers s immediately after $t$. The total instantaneous front speed fluctuation $\delta v_{\mathrm{r}}(t)$ around $v_{N}$ is therefore given by

$$
\delta v_{\mathrm{r}}(t)=\dot{S}_{\mathrm{r}}(t)-v_{N}=\delta v_{\mathrm{r}, \mathrm{mf}}(t)+\delta v_{\mathrm{r}, \mathrm{s}}\left(t_{+}\right) .
$$

Notice that the definition of $\delta v_{\mathrm{r}, \mathrm{s}}\left(t_{+}\right)$in this manner automatically warrants the condition that the average of $\delta v_{\mathrm{r}, \mathrm{s}}\left(t_{+}\right)$[but not $\delta v_{\mathrm{r}}(t)$ ] over all possible collision sequences after $t$ is identically zero. A further average of $\delta v_{\mathrm{r}}(t)$ over an ensemble of realizations at time $t$, however, must yield zero.

The formal expression of $D_{f}$ is then finally obtained using the Green-Kubo formula

$$
D_{f}=\frac{1}{2} \lim _{T \rightarrow \infty} \int_{0}^{T} d t^{\prime}\left\langle\left\langle\delta v_{\mathrm{r}}(t) \delta v_{\mathrm{r}}\left(t+t^{\prime}\right)\right\rangle_{\mathrm{s}}\right\rangle_{\mathrm{r}} .
$$

In Eq. (28), one first needs to average over the random number sequences chosen to update the front after time $t$ (subscript s), and then average over the initial realizations at time $t$ (subscript $\mathrm{r}$ ).

\subsection{The Case of Small Values of $N$}

By now we have seen that the extent of dependence of the front speed on the details of the models increases with decreasing $N$. This in itself is not surprising when one realizes that the relative strength in fluctuations in the number of particles increases with deceasing $N$ and that the fluctuation characteristics are indeed controlled by the specific details of the model. In Sec. 2.6, 
we discuss propagating fronts in discrete particle and lattice systems where the average number of particles $N$ per lattice site in the stable phase of the front is "small". Studies on front propagation at small values of $N$, however, have been limited to only the $\mathrm{X} \leftrightharpoons 2 \mathrm{X}$ and the $\mathrm{X}+\mathrm{Y} \rightarrow 2 \mathrm{X}$ reaction-diffusion systems $[5,14,34,54,55,71-74,80,90,96,116,118,119] .{ }^{37}$ The mean-field description of all the one-dimensional models studied in these references yield the Fisher-Kolmogorov equation on a lattice, given by (with a redefined time scale and in full generality of parameters)

$$
\frac{\partial \phi_{k}}{\partial t}=D\left[\phi_{k+1}+\phi_{k-1}-2 \phi_{k}\right]+r\left(N \phi_{k}-\phi_{k}^{2}\right)
$$

where $r$ is the rate of reaction, $D$ is the rate of diffusion of the particles to their nearest neighbours, and $\phi_{k}=\left\langle\left\langle N_{k}\right\rangle\right\rangle / N$. In the stable phase of these fronts there are $N$ particles per lattice site on average, and in the mean-field limit, the front becomes a pulled front with speed $v^{*}$ (when the space continuum limit is taken, then $\left.v^{*}=2 \sqrt{\operatorname{DrN}}\right)$.

\subsubsection{Summary of the Models Studied and Known Results}

In the various models studied, the value of $N$ varies between $N \ll 1$ to $N \simeq 20$. Section 2.6 is divided into several subsections with progressively reduced value of $N$. One common trend in all these (one-dimensional) lattice models is to identify the front position with the instantaneous position of the foremost particle - do notice that this is identical to the i.f.o.l.s. defined in Sec. 2.5, and therefore, it is not the same as the f.o.l.s. defined in Sec. 2.3.

(i) Reaction-diffusion model $\mathrm{X}+\mathrm{Y} \rightarrow 2 \mathrm{X}$ on a lattice, where both $\mathrm{X}$ and $\mathrm{Y}$ particles can diffuse to their next nearest neighbour lattice sites with the same diffusion rate $D$; any $\mathrm{Y}$ particle coming into contact with an X particle on the same lattice site is instantaneously converted to an X particle [71]: In this model, initially, the entire lattice contains only $\mathrm{Y}$ particles, and one $\mathrm{X}$ particle is introduced at the left end of the lattice at $t=0$. Soon afterwards, a front consisting of $\mathrm{X}$ particles propagates into a region full of $\mathrm{Y}$ particles. The mean-field description of this model yields a reaction rate $r \rightarrow \infty$,

\footnotetext{
${ }^{37}$ In general, any model of discrete particle on a lattice with a small number of particles per lattice site, relatively strong fluctuations render mean-field descriptions inapplicable, and there exist numerous accounts of it in the literature. In the context of reaction-diffusion systems, on general issues of suitability of mean-field descriptions of discrete particle and lattice systems at small number of particles per lattice site, readers may be interested to consult Refs. [6-9,31-33] and the references cited therein, and also references cited in Ref. [71]. The reaction-diffusion systems in these references are of $\mathrm{X} \leftrightharpoons 2 \mathrm{X}, \mathrm{X}+\mathrm{Y} \rightarrow 2 \mathrm{X}, \mathrm{X}+\mathrm{X} \rightarrow \mathrm{X}, \mathrm{X}+\mathrm{X} \rightarrow$ inert and $\mathrm{X}+\mathrm{Y} \rightarrow$ inert type.
} 
resulting in an infinite mean-field front speed. However, in the actual lattice model for $N \rightarrow 0$, this is not the case: dimensional arguments, confirmed by simulation shows that $v_{N} \propto D N$, while the front diffusion coefficient $D_{f}$ is $\propto D$, with proportionality constants of $\mathcal{O}(1)$ [71]. In a discrete-time equivalent of this model, where time is measured in discrete units of $D$ (thereby rendering $D=1$ ) and the system is parallelly updated, the front speed has been shown to behave as $[118,119]$

$$
v_{N}=1-e^{-N / 2},
$$

with $v_{N}$ saturating at 1 for large $N$.

(ii) Reaction-diffusion model $\mathrm{X}+\mathrm{Y} \rightarrow 2 \mathrm{X}$, where on a lattice both $\mathrm{X}$ and $\mathrm{Y}$ particles can diffuse to their next nearest neighbour lattice sites with the same diffusion rate $D$, and a $\mathrm{Y}$ particle coming into contact with an $\mathrm{X}$ particle on the same lattice site is converted to an $\mathrm{X}$ particle with a finite rate $r$ : although one might expect the front speed at small $N$ to have no resemblance with the mean-field expression $v^{*}$, it turns out that for some small value of $N$ and $r \ll 1$, surprisingly the mean-field front speed $v^{*}$ is recovered again [73].

(iii) A reaction-diffusion model similar to the one in (i) above was considered in Ref. [74], with the $\mathrm{X}$ and $\mathrm{Y}$ particles have different diffusion rates, say $D_{X}$ and $D_{Y}$, then for $D_{X} \neq 0$, the front speed depends only on $D_{X}$, and not on $D_{Y}$. At $D_{X}=0$ however, there is no uniformly translating front solution [74].

(iv) With only one particle allowed per lattice site, one of the major variants of the model that has been considered in the literature is the reactiondiffusion system $\mathrm{X} \leftrightharpoons 2 \mathrm{X}$. In this system, only three basic moves are allowed (see Fig. 16): (a) an X particle can diffuse to any one of its neighbour lattice sites with a diffusion rate $D$, provided this neighbouring site is empty; (b) an $\mathrm{X}$ particle can give birth to another one on any one of its empty neighbour lattice site with a birth rate $\varepsilon$; (c) any one of two $\mathrm{X}$ particles belonging to two neighbouring filled lattice sites can get annihilated with a annihilation rate $W$.

For all values of the parameters $D, \varepsilon$ and $W$, there exists an exact relation between the front speed $v_{N}$ and $D_{f}$, namely [90]

$$
\frac{v_{N}}{2}+D_{f}=D+\varepsilon
$$

For $W=0$ and $D / \varepsilon \rightarrow \infty$ [14], and for $W \neq 0$ and $\sqrt{D \varepsilon} /(\varepsilon+W) \rightarrow \infty[81]$, the front speed is given by its mean-field limit, $v^{*}=2 \sqrt{2 D \varepsilon}$, but when $D$ and $\varepsilon$ are of the same order of magnitude, then there are tremendous deviations from this mean-field theory results $[14,54,55]$. In Ref. [55], these differences were studied numerically, and it was shown in [54] that the differences from 
the mean-field theory results can be explained by means of a "self-consistent two-particle approach". For $W \neq 0$, the $W=D$ case was considered in Refs. $[5,34]$ in continuum space, where the front speed is obtained as $v=\varepsilon$ and front diffusion coefficient $D_{f}=D$.

(a)

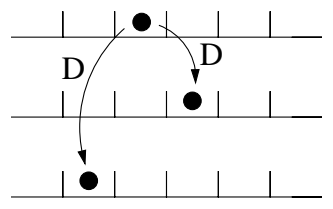

(b)

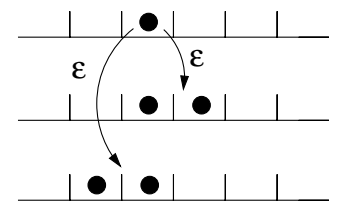

(c)

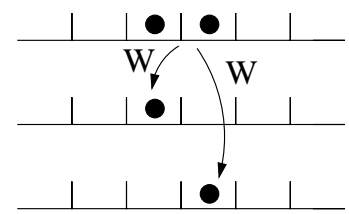

Fig. 16. The microscopic processes that take place inside the system: (a) a diffusive hop with rate $D$ to a neighbouring empty site; (b) creation of a new particle on a site neighbouring an occupied site with rate $\varepsilon$; (c) annihilation of a particle on a site adjacent to an occupied site at a rate $W$.

The full lattice model where $D, W$ and $\varepsilon$ are all different, and $D / W$ and $D / \varepsilon$ are of the same order of magnitude was considered in Ref. [90]; in this case, one has a good approximate solution [90]

$$
N \simeq \frac{\varepsilon}{\varepsilon+W} \quad v_{N} \simeq \frac{\varepsilon(\varepsilon+D)}{\varepsilon+W} \quad \text { and } \quad D_{f} \simeq \frac{(\varepsilon+2 W)(\varepsilon+D)}{2(\varepsilon+W)}
$$

(v) Fluctuating fronts in higher than one spatial dimensions: all the known studies on propagating fronts in discrete particle and lattice realizations in higher than one spatial dimensions have been on $\mathrm{X}+\mathrm{X} \leftrightharpoons 2 \mathrm{X}$ reactiondiffusion system $[80,96,116]$, or on $\mathrm{X}+\mathrm{Y} \rightarrow 2 \mathrm{X}$. We have seen earlier that for fluctuating fronts in one spatial dimension, the front position suffers diffusive wandering around its mean. In higher than one spatial dimensions, the front wandering properties change: it is then possible to formulate the problem along the lines of interfacial growth phenomena $[10,47,53,60,61]$ and study the associated scaling properties. However, there exists tremendous ambiguity regarding how to properly characterize the wandering behaviour of fronts in higher than one dimensions $[80,116]$, although the results indicate that any generic upper critical dimension must be higher than 4 [80].

In the following sections 2.6.2 through 2.6.6, we provide detailed descriptions of points (i) through (v) above, one point in each section.

\subsubsection{Front Propagation in the Diffusion-Limited Irreversible Process $X+Y \rightarrow$ $2 X$}

In Sec. 2.6.2, we elaborate the model introduced in point (i) of Sec. 2.6.1. It was first introduced and studied in the $N \rightarrow 0$ limit in Ref. [71]. In this model, the front position is (naturally) identified by the location of the foremost (rightmost) X particle, and the only way the front (consisting of $\mathrm{X}$ particles) can propagate into the region occupied by the $\mathrm{Y}$ particles is by diffusion. Front 
propagation in this lattice model is therefore diffusion-limited, which results in a front speed $\propto N$, as opposed to an infinite front speed as the mean-field theory predicts.

To solve for front speed and front diffusion, the following dimensional argument was proposed in Ref. [71]: there are only two dimensionful parameters in this problem: $N$, which scales as inverse length, and $D$. Therefore, $\alpha=v_{N} / D N$ is a dimensionless quantity. On the other hand, wandering of the front region is diffusive, and if one denotes the front width due to the diffusive broadening by $w(t)$, then $w^{2}(t) \sim D_{f} t$, and from the definition of $w(t)$ and using the parameters of the problem $N$ and $D$, one can define another dimensionless quantity $\beta=w^{2}(t) / D t \equiv D_{f} / D$. These arguments suggest that $v_{N} \propto D N$ and $D_{f} \propto D$. These conjectures were confirmed in simulation, and the values of $\alpha$ and $\beta$ were numerically obtained to be equal to 1.05 and 3.57 respectively (with uncertainties in the last digits), using $N$ values $0.025,0.05$ and $0.1 .{ }^{38}$

In a continuum space formulation, in the comoving frame of the foremost $\mathrm{X}$ particle $(\xi=N x)$, the probability density $p_{X Y}(\xi)$ that the first Y particle is at a distance $\xi$ on the right of the foremost $\mathrm{X}$ particle, and the probability density $p_{X X}(\xi)$ that the first $\mathrm{X}$ particles on the left of the foremost $\mathrm{X}$ particle were also calculated in Ref. [71]. In this frame, the foremost $\mathrm{X}$ particle is stationary (say at $\xi=0$ ), and the $\mathrm{Y}$ particles in the region $\xi>0$ and the $\mathrm{X}$ particles in the region $\xi<0$ undergo uncorrelated diffusive movements towards $\xi=0$. The density of particles $n(\xi)$ (Y particles for $\xi>0$ and $\mathrm{X}$ particles for $\xi<0$ ) are then obtained from ${ }^{39}$

$$
\frac{\partial n}{\partial t}-v_{N} N \frac{\partial n}{\partial \xi}=2 D N^{2} \frac{\partial^{2} n}{\partial \xi^{2}}
$$

The stationary state solution of this equation with the appropriate boundary conditions then yields

\footnotetext{
${ }^{38}$ Notice that the front really propagates due to the diffusion of $\mathrm{X}$ particles, in the model of Ref. [71], if the two species X and Y have different diffusion rates, then one would simply expect $v_{N}=\alpha D_{X} N$ with the same value of $\alpha$ as deduced above. That this idea is actually correct was verified by simulations [74]. We will return to this point in Sec. 2.6.4.

${ }^{39} \mathrm{In}$ Eq. (33) we need to use the diffusion rate of $\mathrm{X}$ particles for $\xi<0$ (or the diffusion rate of $\mathrm{Y}$ particles for $\xi>0$ ) in the comoving frame of the foremost $\mathrm{X}$ particle. Therefore, these diffusion rates are actually relative diffusion rates of a pair of already diffusive particles, and the value for both of them is $2 D$. Notice however that in Ref. [71], $D$ was used as the relative diffusion rate as opposed to $2 D$. As a result, the solutions of Eq. (33) that we obtain here, which are the correct ones, are not the same as the ones obtained in Ref. [71].
} 


$$
\begin{aligned}
& n_{Y}(\xi>0)=N\left[1-\exp \left(-v_{N} \xi /[2 D N]\right)\right]=N[1-\exp (-\alpha \xi / 2)] \text { and } \\
& n_{X}(\xi<0)=N .
\end{aligned}
$$

From Eq. (34), the probability $\rho(\xi)$ of finding a Y particle at a distance $\xi$ on the right of the foremost $\mathrm{X}$ particle, is obtained from the integral equation $n_{Y}(\xi) / N=\rho(\xi)+\int_{0}^{\xi} \rho\left(\xi^{\prime}\right) d \xi^{\prime}$, leading to the solution $\rho(\xi)=\alpha\left(e^{-\alpha \xi / 2}-\right.$ $\left.e^{-\xi}\right) /(2-\alpha)$. The probability $\theta(\xi)$ of finding a $\mathrm{X}$ particle at a distance $\xi$ on the left of the foremost $\mathrm{X}$ particle, is similarly obtained from the integral equation $n_{X}(\xi) / N=\theta(\xi)+\int_{\xi}^{0} \theta\left(\xi^{\prime}\right) d \xi^{\prime}$, yielding $\theta(\xi)=\alpha e^{-\alpha|\xi| / 2} / 2$. As already mentioned, these solutions are different from the ones obtained in Ref. [71] (see footnote 39).

Notice that Eq. (33) does not determine the front speed in any way, instead $v_{N}$ enters as an input for the solution of $\rho(\xi)$ and $\theta(\xi)$. At best, $v_{N}$ has to be determined from somewhere else. In a model closely related with the the one analyzed in Ref. [71], where time was discretized in units of $D^{-1}$, this incompleteness was removed: in the $N \rightarrow 0$ limit, $v_{N}$ was deduced from first principles to be equal to $N / 2$ at the leading order of $N[118,119]$. In this model, in a unit time, each particle moves one lattice distance randomly towards its right or left, and the method of "parallel updating" 40 was employed in the simulation algorithm. The argument to derive the expression of front speed in this model in the $N \rightarrow 0$ limit is the following: the front speed is essentially determined by considering the two foremost $\mathrm{X}$ particles (which are separated by an average of $1 / N$ lattice sites). ${ }^{41}$ In fact, the only non-zero contribution to the front speed occurs when the second $\mathrm{X}$ particle is on the lattice site just behind the foremost one, or on the same lattice site as the foremost one (see footnote 42 for an explanation). In the latter case, one of the $\mathrm{X}$ particles at the location of the front is tagged to be the foremost $\mathrm{X}$ particle and the other is then tagged as the second X particle. In the comoving frame of the front, let us tag the location of the foremost $\mathrm{X}$ particle by $k=0$, and denote the probability that the second $\mathrm{X}$ particle is $k$ lattice sites behind the foremost $\mathrm{X}$ particle by $p_{-k}$. It is then easily seen that ${ }^{42}$

\footnotetext{
40 The idea behind parallel updating in this model is that in a unit time, all particles move diffusively one lattice distance randomly either towards their left or the right, and when this update brings an $\mathrm{X}$ particle in contact with any number of $\mathrm{Y}$ particles on a lattice site, all the $\mathrm{Y}$ particles on that lattice site are instantaneously converted to $\mathrm{X}$ particles.

${ }^{41}$ In the limit $N \rightarrow 0$, the role of a third (or more) particle near the foremost $\mathrm{X}$ particle can be neglected; they only provide higher order corrections.

${ }^{42}$ Equation (35) is obtained by noticing that in a unit time, the two foremost $\mathrm{X}$ particles can execute a total of four moves, each with probability $1 / 4$. When the two
} 


$$
v_{N}=\left[\frac{p_{-1}}{4}+\frac{p_{0}}{2}\right]
$$

The steady state solution of $p_{-1}$ and $p_{0}$ are obtained from the master equation for $p_{k}$ 's around foremost $\mathrm{X}$ particle (i.e., $k=-2,-1, \ldots, 2$ etc.), yielding ${ }^{43}$

$$
p_{-1}=N, \quad p_{0}=N / 2 \quad \text { and } \quad v_{N}=N / 2 .
$$

The above derivation clearly does not hold for higher values of $N$, as one needs to consider the case where a lattice site is occupied by multiple number (many more than two) of X particles. Such an analysis was also presented in Ref. $[118,119]$, in which, having denoted the probability of having the $k$-th lattice site occupied by $n_{k}$ number of particles by $p\left(n_{k}\right)$, one has [Eq. (37) is obtained by following the logic of footnote 42]

$$
\begin{aligned}
& P_{-}=\sum_{n_{0}=1}^{\infty} \sum_{n_{-1}=1}^{\infty} \frac{p\left(n_{0}, n_{-1}\right)}{2^{n_{0}+n_{-}}}, \quad P_{+}=\sum_{n_{0}=1}^{\infty}\left[1-\frac{1}{2^{n_{0}}}\right] p\left(n_{0}\right) \quad \text { and } \\
& P_{0}=\sum_{n_{0}=1}^{\infty} \sum_{n_{-1}=1}^{\infty} \frac{1}{2^{n_{0}}}\left[1-\frac{1}{2^{n_{-1}}}\right] p\left(n_{0}, n_{-1}\right)
\end{aligned}
$$

respectively for the probability of the front to step backward one lattice site, to remain where it presently is, and to step forward one lattice site in one unit time. To obtain the front speed from Eq. (37) however, one needs the probability distributions $p\left(n_{0}\right)$ and $p\left(n_{-1}\right)[118,119]$. In Ref. [118,119], while $p\left(n_{-1}\right)$ was obtained from the simulations as a Poissonian with average $N$, a truncated Poissonian ${ }^{44}$ was used for $p\left(n_{0}\right)$, yielding the result [notice that one recovers Eq. (36) for $v_{N}$ from Eq. (38) in the limit $N \rightarrow 0$ ]

foremost X particles are separated by one lattice site, then the front moves forward one lattice distance with probability $1 / 2$, moves backward one lattice distance with probability $1 / 4$ and remains stationary with probability $1 / 4$. This explains the origin of the $p_{-1} / 4$ term. On the other hand, when the two foremost $\mathrm{X}$ particles on the same site, then the front moves forward one lattice distance with a probability $3 / 4$, and moves backward one lattice distance with a probability $1 / 4$ - giving rise to the $p_{0} / 2$ term. When the second $\mathrm{X}$ particle is more than one lattice site behind the foremost $\mathrm{X}$ particle, then the front moves forward or backward by one lattice distance with equal probabilities $1 / 2$, which is why $p_{-k}$ for $k>1$ does not contribute in Eq. (35).

${ }^{43}$ For the master equation and its solution, we urge the readers to consult Ref. $[118,119]$; although here we point out the similarity between Eqs. (36) and (34) in both of these (very similar) models, the density of X particles behind the foremost one is $N$.

${ }^{44}$ The truncated Poissonian distribution for the probability of $n_{0}$ number of particles at $k=0$ is given by $p_{T}\left(n_{0}\right)=\left(1-\delta_{n_{0}, 0}\right)\left(1-e^{-N}\right)^{-1} n_{0} e^{-n_{0}}$. 


$$
v_{N}=1-e^{N / 2}
$$

The agreement of the front speed with simulation results $[118,119]$ is shown in Fig. 17.

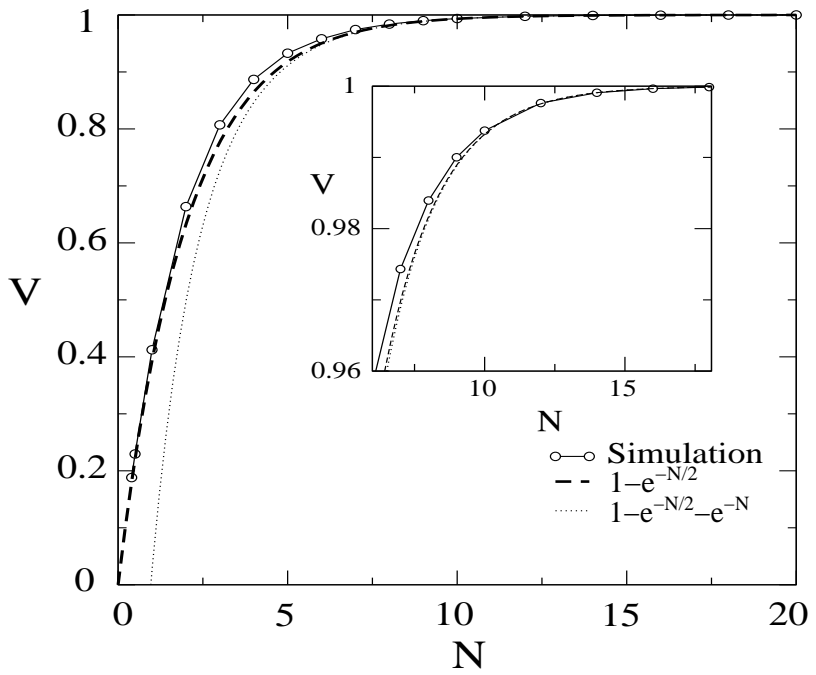

Fig. 17. Equation (38) vs. simulation data for front speed, adopted from Ref. [118, 119]. Here $v$ should be read as $v_{N}$. The dotted curve shows the agreement between theory and simulation if one uses a Poissonian distribution also for $p\left(n_{0}\right)$. Notations have been modified from the original to maintain consistency with the text.

\subsubsection{Reaction-diffusion Model $X+Y \rightarrow 2 X$ at Small $N$ with Reaction Rate $r \rightarrow 0$}

In Ref. [73], the reaction-diffusion model $\mathrm{X}+\mathrm{Y} \rightarrow 2 \mathrm{X}$ was numerically studied at small values of $N,(N=0.1,0.3,1,5$ and 10$)$ and reaction rates $r \ll 1$ $(r \leq 0.02)$. The details of the microscopic model are already described in point (ii) of Sec. 2.6.1.

With the front position identified by the position of the foremost $\mathrm{X}$ particle, the front speed at different values of $N$ and as a function of reaction rate $r$ is shown in Fig. 18 ( $p$ and $c$ should be read as $r$ and $N$ respectively), where $\nu(r)=v_{N}(r) / 2 \sqrt{D N}$. If the mean-field theory were applicable in this model, then $\nu(r)$ should scale as $\sqrt{r}$ (dashed curve of Fig. 18). Figure 18 reveals that typically most values of $N$ yield strong deviation from mean-field result for $\nu(r)$, except for $N=10$ for all values of $r$. Reference [73] also claimed that the trend below $r \simeq 0.001$ indicates that the mean-field result front speed for $r \lesssim 0.001$, however there are too few data points below $r \simeq 0.001$ in Fig. 18 to arrive at a definitive conclusion.

No theoretical argument for why the $N=10$ data for the front speed follows the mean-field theory results so well was provided in Ref. [73] (neither is it 


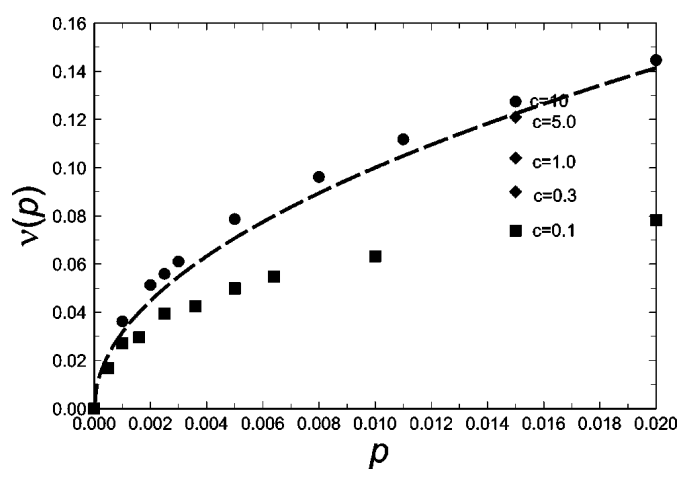

Fig. 18. Front speed at different values of $N$ and as a function of reaction rate $r(p$ and $c$ should be read as $r$ and $N$ respectively), where $\nu(r)=v_{N}(r) / 2 \sqrt{D N}$. Dashed curve: mean-field result $\nu(r)=\sqrt{r}$.

clear from a simple argument). Moreover, since Fig. 18 indicates that the mean-field results are closely followed for $N=10$, it is a natural extension to investigate, by means of simulations, if the front profile also follows the meanfield result. However, this point was not investigated in Ref. [73]. Instead, the front profile was investigated for $N=0.1$, and from the plots that were provided in Ref. [73], the front profile seems to behave as

$$
\phi(\xi)=a \exp \left\{-b \exp \left[\frac{\left(\xi-\xi_{0}\right)^{2}}{2 \sigma^{2}}\right]\right\} .
$$

The plots that were supplied in Ref. [73] in support of Eq. (39) are shown in Fig. 19. Figure 19(a) shows the front the front profile (here $A$ should be read as $\phi(\xi)$ and $x$ should be read as $\xi$ ) for three values of the reaction rates $r=0.0025,0.005$ and 0.01 (left above from left to right). The dashed lines in Fig. 19(a) correspond to Eq. (39) with numerically determined $a, b$ and $\sigma$. The plots of $\beta(\xi)=\sqrt{\log _{10}\left|\log _{10}[\phi(\xi)]\right|}$ are shown in Fig. 19(b): the right above left to right plots supposedly correspond to $r$ values $0.0025,0.005$ and 0.01 respectively.

\subsubsection{The Case of Different Diffusion Rates for $X$ and $Y$ Particles in the Model of Point (i)}

In Ref. [74], a model similar to the one proposed in Ref. [71] was studied. In this model, the two species $\mathrm{X}$ and $\mathrm{Y}$ particles have two different diffusion rates, namely $D_{X}$ and $D_{Y}$. Both the cases $D_{X} \neq 0$ and $D_{X}=0$ were considered in the ensuing analysis. The lattice is initially filled with $\mathrm{Y}$ particles with an average number of particles $N$ per lattice site and at $t=0$, an $\mathrm{X}$ particle is introduced at the leftmost lattice site, just like the model discussed in point (i) of Sec. 2.6.1. However, only one particle is allowed per lattice site in this model, and when two neighbouring lattice sites are occupied by one $X$ and one 

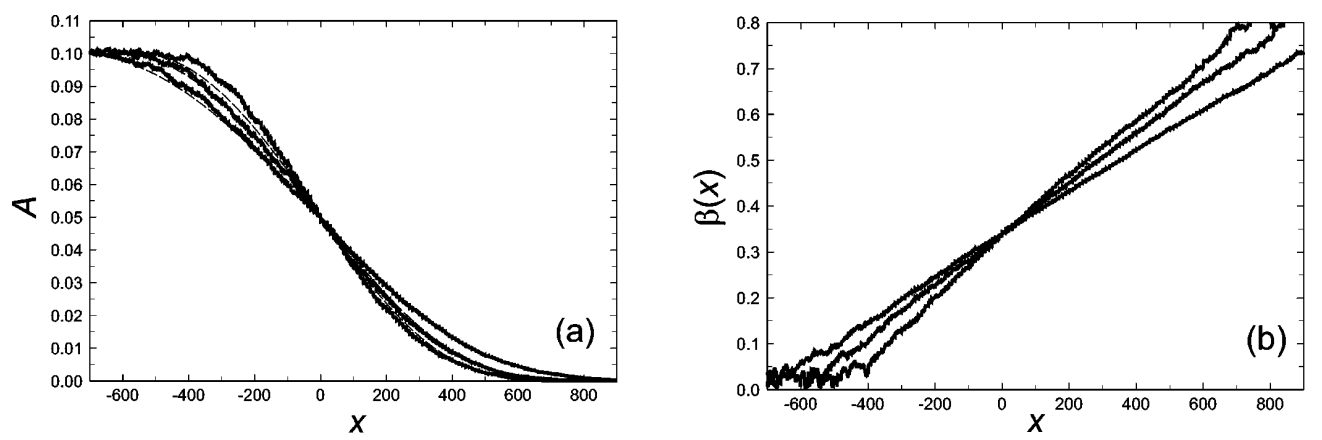

Fig. 19. Plots in support of Eq. (39): (a) front the front profile (here $A$ should be read as $\phi(\xi)$ and $x$ should be read as $\xi$ ) for three values of the reaction rates $r=0.0025,0.005$ and 0.01 (left above from left to right). The dashed lines correspond to Eq. (39) with numerically determined $a, b$ and $\sigma$. (b) Plots of $\beta(\xi)=\sqrt{\log _{10}\left|\log _{10}[\phi(\xi)]\right|}$ are shown in Fig. 19(b): the right above left to right plots supposedly correspond to $r$ values $0.0025,0.005$ and 0.01 respectively.

$Y$ particle, the $Y$ particle is instantaneously converted to an $X$ particle. As pointed out in Ref. [74], the restriction that only one particle is allowed per lattice site is not necessary, other than when $D_{X}=0$.

In this model, the $D_{X} \neq 0$ does not reveal any surprises (see footnote 38 [74]). Moreover, the density profile of $\mathrm{Y}$ particles on the right of the foremost $\mathrm{X}$ particle (located at $\xi=0)$ in a continuum-space formulation $(\xi=N x)$ turns out to be $n_{Y}(\xi)=N\left[1-\exp \left\{-v_{N} \xi /\left[\left(D_{X}+D_{Y}\right) N\right]\right\}\right][74]$ (see Fig. 20) 一 this is also consistent with Eq. (34).

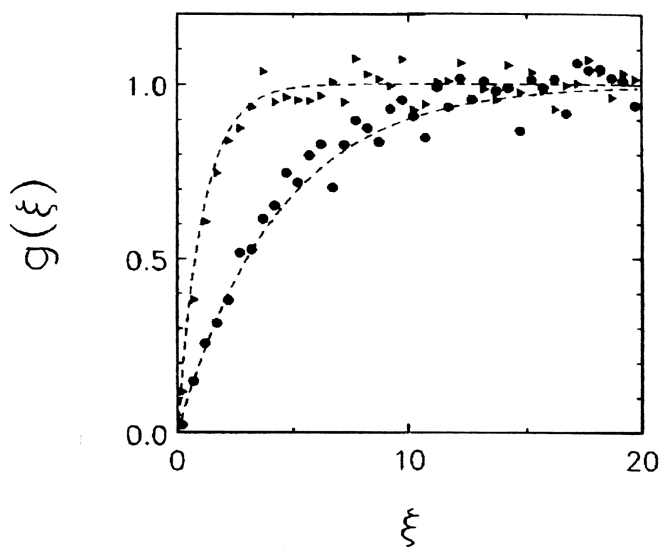

Fig. 20. Comparison of the density profile of $\mathrm{Y}$ particles on the right of the foremost $\mathrm{X}$ particle in a continuum-space type theory $g(\xi)=n_{Y}(\xi) / N=$ $1-\exp \left\{-v_{N} \xi /\left[\left(D_{X}+D_{Y}\right) N\right]\right\}$ and simulation data for two different $N$ values. Triangles: $D_{X}=0.2, D_{Y}=0.8$, circles: $D_{X}=0.8, D_{Y}=0.2$.

The case of $D_{X}=0$, however, is more complicated as we explain below. In this case, the front propagates only due to the diffusive flux of $Y$ particles towards the foremost $\mathrm{X}$ particle, and at any time $t$, with the front position 
defined by the location $x_{f}(t)$ of the foremost $\mathrm{X}$ particle in a continuum-space formulation, one has [74]

$$
v_{N}(t)=-\left.D_{Y} \frac{\partial n_{Y}(x, t)}{\partial x}\right|_{x_{f}(t)}
$$

while the density profile of $\mathrm{Y}$ particles on the right of the foremost $\mathrm{X}$ particle is obtained from the analogous form of Eq. (33) as (notice that the rate of diffusion of the $\mathrm{Y}$ particles towards the foremost $\mathrm{X}$ particle is $\left.D_{Y}\right)^{45}$

$$
\frac{\partial n}{\partial t}-v_{N} \frac{\partial n}{\partial x}=D_{Y} \frac{\partial^{2} n}{\partial x^{2}}
$$

Equations (40) and (41) together are then solved with the ansatz $n_{Y}(x, t)=$ $N g\left\{\left[x-x_{f}(t)\right] / \sqrt{D_{Y} t}\right\}$ and a yet unknown function $g$ [74]. This ansatz actually suggests that $x_{f}(t) \sim \sqrt{t}$, and that the front speed $v_{N}(t) \sim t^{-1 / 2}$ is actually time-dependent. At low density of particles per lattice site $N$, the solution of $n_{Y}(x, t)$ turns out to be the error function $g\left\{\left[x-x_{f}(t)\right] / \sqrt{D_{Y} t}\right\}=\operatorname{erf}\{[x-$ $\left.\left.x_{f}(t)\right] /\left[2 \sqrt{D_{Y} t}\right]\right\}$. These aspects were verified in Ref. [74] (see Fig. 18).
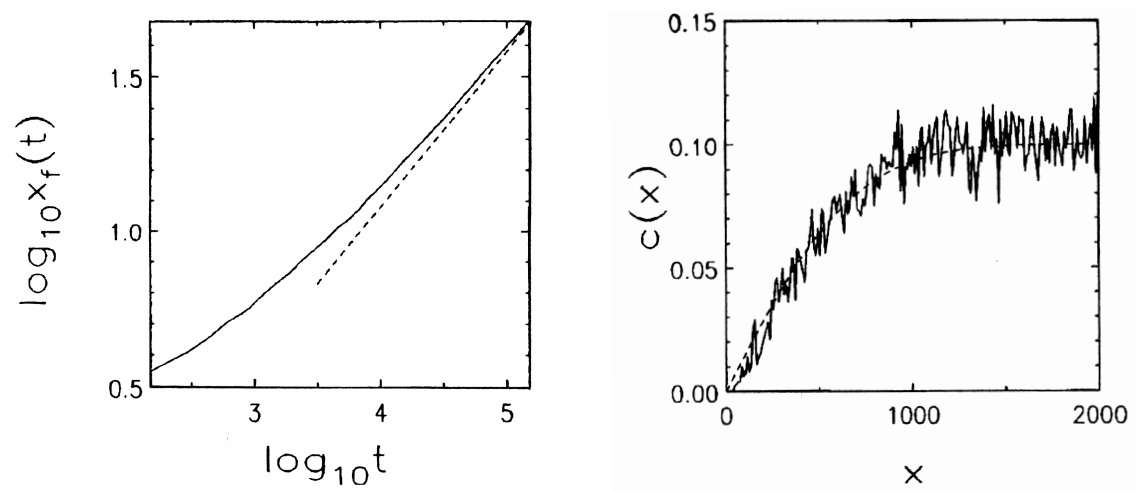

Fig. 21. The plots of $x_{f}(t)$ vs. $t$ in $\log -\log$ scale, and that of $n_{Y}(x, t)$ at $t=10000$ units. Here $c(x)$ should be read as $n_{Y}(x)$. Dashed lines correspond to $x_{f}(t) \sim \sqrt{t}$ and Eq. (41) respectively.

\footnotetext{
${ }^{45}$ One must be aware of the fact that the usage of $D_{Y}$ as the rate of diffusion of the $\mathrm{Y}$ particles towards the foremost $\mathrm{X}$ particle is valid at a low density of $\mathrm{Y}$ particles. In this model, which allows only one particle per site, the diffusion of a $\mathrm{Y}$ particle is permitted only if its neighbouring lattice sites are empty. Strictly speaking, therefore, to obtain the the rate of diffusion of the $\mathrm{Y}$ particles towards the foremost $\mathrm{X}$ particle one should also take into account probability of having two next-nearest neighbour Y particles. At low density of Y particles this probability can be neglected, permitting the use of $D_{Y}$ in Eq. (41).
} 


\subsubsection{Reaction-diffusion Model $X \leftrightharpoons 2 X$ with Only One Particle Allowed per Lattice Site}

In Sec. 2.6.5, we discuss the model of point (iv) of Sec. 2.6.1. Below, up to Eq. (46) we present the general features of this model following Refs. $[14,54,55,90]$, and then we discuss the results in different parameter regimes.

Like in the other subsections of Sec. 2.6, for all values of $D, \varepsilon$ and $W$, we define the position of the front by the location of the foremost particle. For a given realization, let us denote its position at time $t$ by $k_{f}(t)$. For an ensemble of front realizations, having denoted the probability distribution for the foremost particle to be at lattice site $k_{f}$ by $P_{k_{f}}(t)$, its evolution is then described by

$$
\begin{aligned}
\frac{d P_{k_{f}}}{d t}=(D+\varepsilon) P_{k_{f}-1}+\left[D P_{k_{f}+1}^{\mathrm{empty}}+W P_{k_{f}+1}^{\mathrm{occ}}\right] & \\
& -(D+\varepsilon) P_{k_{f}}-\left[D P_{k_{f}}^{\mathrm{empty}}+W P_{k_{f}}^{\text {occ }}\right] .
\end{aligned}
$$

Here $P_{k_{f}}^{\text {occ }}(t)$ and $P_{k_{f}}^{\text {empty }}(t)$ denote the joint probabilities that the foremost particle is at site $k_{f}$ and that the site $k_{f}-1$ is occupied or empty, respectively. Clearly, $P_{k_{f}}(t)=P_{k_{f}}^{\text {occ }}(t)+P_{k_{f}}^{\text {empty }}(t)$, and $\sum_{k_{f}} P_{k_{f}}(t)=1$. The first term on the r.h.s. of Eq. (42) describes the increase in $P_{k_{f}}(t)$ due to the advancement of a foremost occupied lattice site from position $k_{f}-1$, while the second term describes the increase in $P_{k_{f}}(t)$ due to the retreat of a foremost occupied lattice site from position $k_{f}+1$. The third and the fourth terms, respectively, describe the decrease in $P_{k_{f}}(t)$ due to the advancement and retreat of a foremost occupied lattice site from position $k_{f}$.

From the definition of $P_{k_{f}}(t)$, the mean position and the width of the distribution for the positions of the foremost occupied lattice sites are defined as $x(t)=\sum_{k_{f}} k_{f} P_{k_{f}}(t)$ and $\mathcal{W}^{2}(t)=\sum_{k_{f}}\left[k_{f}-x(t)\right]^{2} P_{k_{f}}(t) .{ }^{46}$ The mean speed and diffusion coefficient of the front are thus given in terms of these quantities as the $t \rightarrow \infty$ limit of $v_{N}=d x(t) / d t$ and $\mathcal{W}^{2}(t)=2 D_{f} t$ - see Fig. 16(c). To obtain them, we need the expressions of $P_{k_{f}}^{\text {occ }}(t)$ and $P_{k_{f}}^{\text {empty }}(t)$. To start with, we have

$$
P_{k_{f}}^{\text {occ }}(t)=\rho_{k_{f}-1} P_{k_{f}}(t),
$$

where $\rho_{k_{f}-1}$ is the conditional probability of having the $\left(k_{f}-1\right)$ th lattice site occupied (the foremost particle is at the $k_{f}$ th lattice site). The set of con-

\footnotetext{
${ }^{46}$ One can also define the foremost occupied lattice site for a realization as the one on the right of which no lattice site has ever been occupied before, and obtain the front speed from this definition following Sec. 2.3. Both of them of course yield the same result due to the $t \rightarrow \infty$ limit.
} 
ditional occupation densities $\rho_{k_{f}-m}$ for $m \geq 1$ can be thought of as determining the front profile in a frame moving with each front realization. For obtaining $v_{N}$ and $D_{f}$, we simply need to know the asymptotic long-time limit $\rho_{k_{f}-1}(t \rightarrow \infty)$, which from here on we will denote simply as $\rho_{k_{f}-1}$. Given $\rho_{k_{f}-1}$, it is then straightforward to obtain from Eq. (42) and the conditions $P_{k_{f}}(t)=P_{k_{f}}^{\text {occ }}(t)+P_{k_{f}}^{\text {empty }}(t)$ and $\sum_{k_{f}} P_{k_{f}}(t)=1$

$$
\begin{gathered}
v_{N}=\frac{d x}{d t}=\varepsilon-\rho_{k_{f}-1}(W-D) \quad \text { and } \\
\frac{d \mathcal{W}^{2}}{d t}=2 D+\varepsilon+\rho_{k_{f}-1}(W-D)
\end{gathered}
$$

Of these, the second equation indicates that the front wandering is diffusive, and an expression of the front diffusion coefficient $D_{f}$ is therefore given by

$$
D_{f}=\frac{1}{2}\left[2 D+\varepsilon+\rho_{k_{f}-1}(W-D)\right]
$$

Note that if we use Eq. (43) in Eq. (42), the latter equation has the form of the master equation for a single random walker on a chain. Thus we can think of the foremost particle as executing a biased random walk, and $D_{f}$ as the effective diffusion coefficient of this walker. Moreover, if we eliminate $\rho_{k_{f}-1}$ from Eqs. (44) and (45), we get the following exact relation

$$
v_{N} / 2+D_{f}=D+\varepsilon
$$

With this background in mind for all values of $D, \varepsilon$ and $W$, we now explore the results in different parameter regimes.

The mean-field limit of this model effectively yields some form of FisherKolmogorov equation. The applicability of the mean-field result, however, is confined only to large values of the diffusion coefficient (while keeping the other parameters $\varepsilon$ and $W$ finite). The need to have large diffusion coefficient is not very difficult to understand intuitively — notice that although in principle there are three parameters in the problem, effectively there are only two of them, since the third one simply sets the time scale. Let us choose these two parameters as $D / \varepsilon$ and $D / W$. The quantity $\ell_{D}=\min (\sqrt{D / \varepsilon}, \sqrt{D / W})[81]$ then can be seen to define a correlation volume, i.e., a length scale over which the position of a particle varies in a unit time. When $\ell_{D} \rightarrow \infty$, the correlations involving two or more particles within a finite range of lattice sites get washed out, and then a mean-field description of the problem becomes suitable.

Having defined $x=k / \sqrt{2 D}$, for $W=0$ and large values of $D / \varepsilon$, a mathematical derivation of the mean-field limit of this model in the form of the 
Fisher-Kolmogorov equation

$$
\frac{\partial \phi}{\partial t}=\frac{1}{2} \frac{\partial^{2} \phi}{\partial x^{2}}+2 \varepsilon\left(\phi-\phi^{2}\right)
$$

can be found in Ref. [14] (also see the references cited therein). ${ }^{47}$ While we leave the interested readers on their own for this mathematical argument, here we concentrate on the Refs. $[54,55]$ that investigated, by means of a "self-consistent two particle model", the role of interparticle correlations in the approach of the front speed towards its mean-field value ${ }^{48} v^{*}=2 \sqrt{2 D \varepsilon}$ for increasing value of $D / \varepsilon$. We make an important observation here that this approach only works for $W=0$ (we will return to this point later).

The first step of this "self-consistent two particle model" is to derive the front speed for small $D / \varepsilon$ at $W=0$, and it appears already in [14]: the idea is that far behind the foremost particle, the density of particles approach the homogeneous equilibrium density $N: \lim _{m \rightarrow \infty} \rho_{k_{f}-m}=N$. From the master equation of the microscopic processes for $W=0$, it is easy to show that the homogeneous equilibrium solution for the total probability is of product form (so that the probability of having different sites occupied is uncorrelated), and that the equilibrium occupation density $N$ is simply given by $N=1$. ${ }^{49}$ Then the crudest approximation for the front profile $\rho_{k_{f}-m}$ and in particular for $m=1$ is just to take $\rho_{k_{f}-1} \approx N$. Substitution of this approximation into Eq. (44) then immediately yields $[14,54,55]$

$$
v_{N}=\varepsilon+D
$$

Equation (47) agrees well with the simulations in the limit $D / \varepsilon \rightarrow 0$.

While Ref. [55] is mostly about numerical studies of how the mean-field result for front speed $v^{*}=2 \sqrt{2 D \varepsilon}$ is obtained from the first half of Eq. (44) in the limit $D / \varepsilon \rightarrow \infty$, the transition from the front speed in Eq. (47) to $v^{*}$

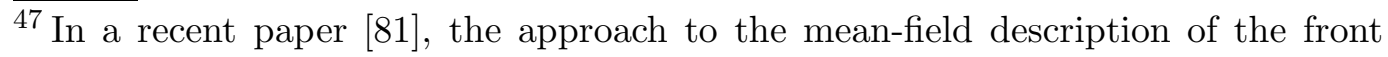
has been investigated in the light of Eq. (11). The idea is to define a quantity $N^{*}=\sqrt{D \varepsilon} /(\varepsilon+W)$ such that for $N^{*} \rightarrow \infty$ (which happens for finite $N$ and $\ell_{D} \rightarrow \infty$ ), not only does one recover the Fisher-Kolmogorov equation, but also for $N^{*} \gg 1$, one expects to observe $v^{*}-v_{N} \sim 1 / \ln ^{2} N^{*}$ and $D_{f} \sim 1 / \ln ^{3} N^{*}$. In other words, Ref. [81] has essentially shown that $N^{*}$ simply plays the same role as $N$ does in Secs. 2.2-2.5.

48 The mean-field Fisher-Kolmogorov equation (47) has front speed $2 \sqrt{\varepsilon}$. When the distance is measured in terms of $k$ and not $x$, this means that the actual $v^{*}$ is $2 \sqrt{2 D \varepsilon}$.

${ }^{49}$ For $W \neq 0, N$ becomes $\varepsilon /(\varepsilon+W)[90]$; we will return to this case later in Sec. 2.6.5.
} 
for increasing values of $D / \varepsilon$ in analyzed by theoretical means in Ref. [54]. What Ref. [54] does is to develop a theory for evaluating $\rho_{k_{f}-1}$, by means of considering the probability distribution $p_{j}$ of having $j$ empty sites between the foremost particle and the second foremost particle, and $\rho_{k_{f}-1}$ is then simply obtained as $\rho_{k_{f}-1}=p_{0}$. The task of obtaining $p_{0}$ itself, however, is not easy, and it is obtained from solving the master equation for the steady state solution of $\left\{p_{j}\right\}$, as we outline below.

With the usage of the normalization $\sum_{j} p_{j}=1$, the master equation for $p_{j}$ is given by

$$
\begin{array}{ll}
\dot{p}_{j}=2 D p_{j-1}+(2 D+\varepsilon) p_{j+1}-(4 D+3 \varepsilon) p_{j} & \text { for } j>0 \text { and } \\
\dot{p}_{0}=(2 D+\varepsilon) p_{1}+2 \varepsilon-2(D+\varepsilon) p_{0} . &
\end{array}
$$

Equation (49) is obtained from the following considerations: the $j>0$ state can be obtained from the $j-1$ state by a forward diffusion of the foremost particle, or by the backward diffusion of the second foremost particle, giving rise to the $2 D p_{j-1}$ term. The $j>0$ state can also be obtained from $(j+1)$ state by a backward diffusion of the foremost particle, or the forward diffusion of the second foremost particle, or by the growth of another particle just on the right of the second foremost particle - this gives rise to the $(2 D+\varepsilon) p_{j+1}$ term. Any $j>0$ state contributes not only to $j+1$ and $j-1$ states by the reverse processes, but also to the $j=0$ state when a particle is created on either side of the foremost particle, which finally explains the $(4 D+3 \varepsilon) p_{j}$ term. The $(2 D+\varepsilon) p_{1}$ is obtained from any $j>0$ state by the creation of a particle on either side of the foremost particle. In addition, the $j=0$ state from the $j=1$ state by a creation of a particle on the right of the second foremost particle, by a forward diffusion of the second foremost particle or by a backward diffusion of the foremost particle. On the other hand, state $j=0$ contributes only to $j=1$ state when the second foremost particle diffuses backward or the foremost particle diffuses forward. When all these are properly taken into account along with the normalization condition $\sum_{j} p_{j}=1$, the second half of Eq. (48) is obtained immediately. The steady state of Eq. (48) is then easily solved by the ansatz $p_{j}=p_{0}\left(1-p_{0}\right)^{j}$ for $\forall j>0$ [54], yielding

$$
p_{0}=\frac{\sqrt{9 \varepsilon^{2}+16 D \varepsilon}-\varepsilon}{2(2 D+\varepsilon)} .
$$

The lesson one learns from this clever approach is actually quite instructive. Notice that Eq. (49) automatically yields the proper $D / \varepsilon \rightarrow 0$ limit of the front speed (48). Its $D / \varepsilon \rightarrow \infty$ behaviour however yields $v_{N}=\sqrt{D \varepsilon}$, which is a factor of $\sqrt{8}$ smaller than $v^{*}=2 \sqrt{2 D \varepsilon}$. This itself should not strike one as surprising. Instead, with the observation that the involvement of a third 
foremost particle on the left of the second one is always ignored (in the sense that the site on the left of the second foremost particle is always assumed to be empty), the fact that even in this crude form this formalism at least provides the $\sqrt{D \varepsilon}$ behaviour of $v_{N}$ at large $D / \varepsilon$ is surprising.

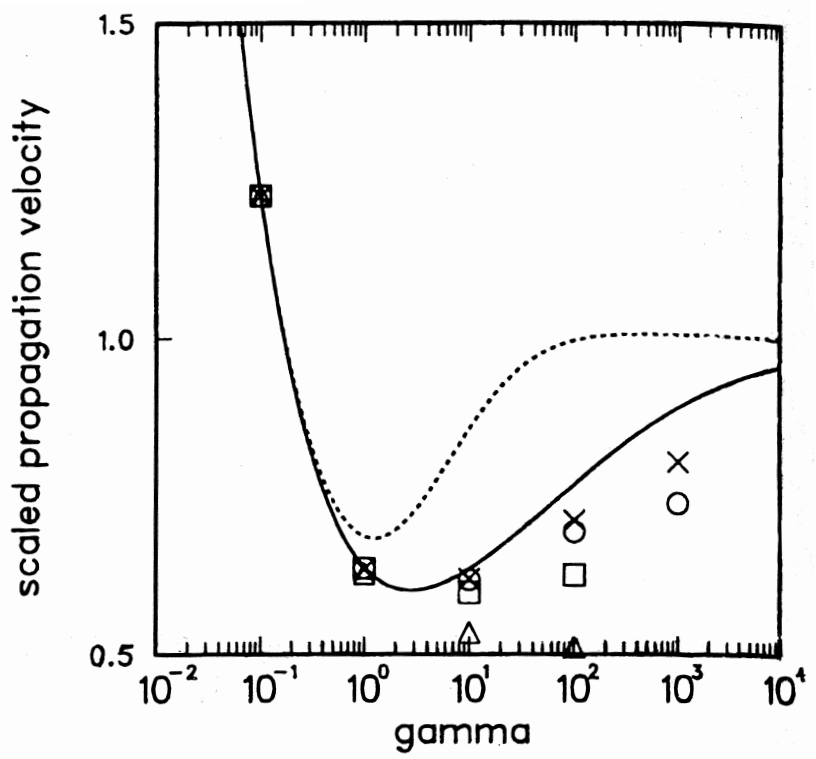

Fig. 22. $v_{N} / v^{*}$ for $\varepsilon=2$ and $\gamma=2 D$ : simulations (crosses), "correlated two-particle self-consistent approach" (solid curve), and "uncorrelated $n$-particle approach" with $n=3$ (open triangles), 5 (open squares) and 10 (open circles). Also shown is the ad hoc functional dependence $v_{N}=\left[1+\gamma(1+\gamma / 8)^{-1 / 2}\right] / 2$ of $v_{N} / v^{*}$ on $\gamma$ by the dotted curve.

For a remedy of this drawback, Ref. [54] also invokes a "correlated two-particle self-consistent approach", in which diffusive moves of the second foremost particle are prevented due to the presence of the third foremost particle just next to the second one. With $p_{a}$ as the probability of having the second and the third foremost particles next to each other, the probability of the second foremost particle making a diffusive hop towards the left is reduced by $D(1-$ $\left.p_{a}\right)$. Reference [54] then obtains the front speed as a function of increasing $D / \varepsilon$ by means of an effective functional dependence of $p_{a}$ on $p_{0}$, namely $p_{a}=$ $B p_{0}-(B-1) p_{0}^{2}$, where the parameter $B$ is chosen in such a way that it leads to $v_{N} \rightarrow v^{*}$ for $D / \varepsilon \rightarrow \infty$. However, in this process, an analytical form of $v_{N}$ as a function of $D / \varepsilon$ is not obtained, although a predicted value of $v_{N}$ can be numerically obtained for a given value of $D / \varepsilon$. In Fig. 22, we present the graph from Ref. [54] for $\varepsilon=2$ and $\gamma=2 D$. It shows all the results for $v_{N} / v^{*}$ together: simulations (crosses), "correlated two-particle self-consistent approach" (solid curve), and "uncorrelated $n$-particle approach" with $n=3$ (open triangles), 5 (open squares) and 10 (open circles). Also shown in Fig. 22 is the ad hoc functional dependence $v_{N}=\left[1+\gamma(1+\gamma / 8)^{-1 / 2}\right] / 2$ of $v_{N} / v^{*}$ on $\gamma$ (adopted because of its simple functional dependence having proper limits 
at $D / \varepsilon \rightarrow 0$ and $D / \varepsilon \rightarrow \infty)$ by the dotted curve.

Having thus concluded the $W=0$ cases studied in the literature in the above paragraph, we now embark on the $W \neq 0$ case for this model. The first case of $W \neq 0$, namely $W=D$, has been analyzed in Refs. [5] and [34]. Notice from Eq. (44) that with $W=D$, one does not require to evaluate $\rho_{k_{f}-1}$ any longer - the front speed and front diffusion are simply given by $v_{N}=\varepsilon$ and $D_{f}=D+\varepsilon / 2[5,34]$.

Clearly enough, when $W \neq 0$ but $W \neq D$, one needs to evaluate $\rho_{k_{f}-1}$. Reference [90] considered this case, but only with all parameters of the same order of magnitude. In this case, having estimated $\rho_{k_{f}-1}$ by $N=\lim _{m \rightarrow \infty} \rho_{k_{f}-m}$ (see footnote 49), one obtains [90]

$$
v_{N}=\frac{\varepsilon(\varepsilon+D)}{\varepsilon+W} \text { and } \quad D_{f}=\frac{(\varepsilon+2 W)(D+\varepsilon)}{2(\varepsilon+W)} .
$$

The comparison of Eq. (51) with stochastic simulation data for $D=\varepsilon=0.25$
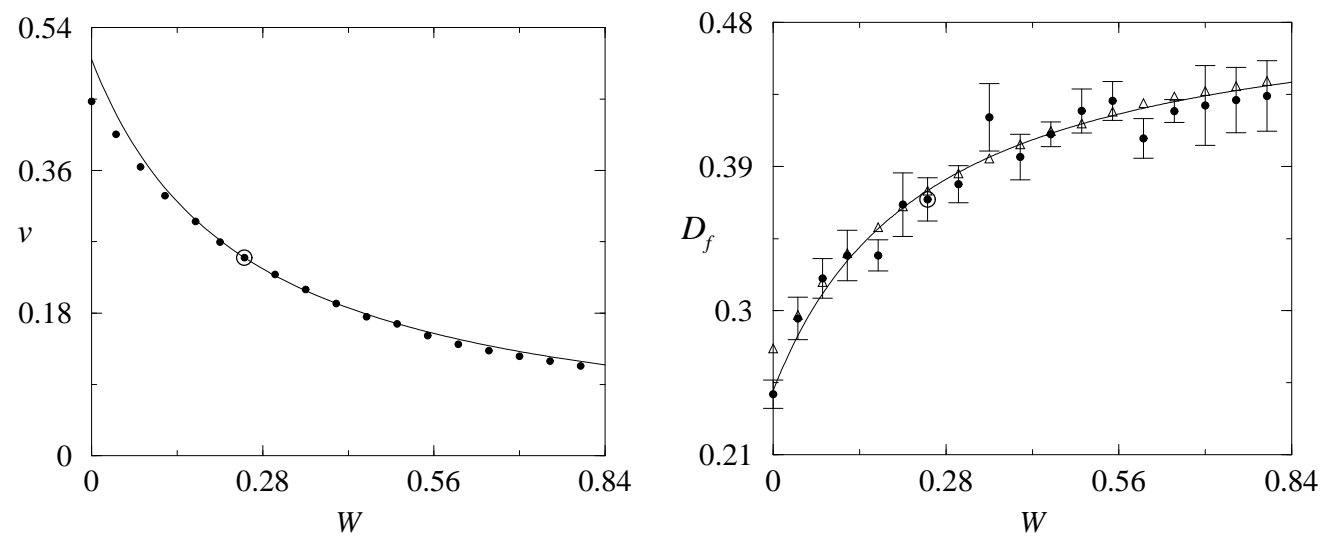

Fig. 23. Comparison of Eq. (51) with stochastic simulation data for $D=\varepsilon=0.25$ are presented in Fig. 23 as a function of $W$ for $D=\varepsilon=0.25$. The larger open circles in the two graphs denote the results of $D=W$ case analyzed in Refs. [5, 34].

are presented in Fig. 23 as a function of $W$ for $D=\varepsilon=0.25$. The larger open circles in the two graphs of Fig. 23 denote the results of $D=W$ case analyzed in Refs. [5,34]. Notice that close to $W=0$ and at larger values of $W$, the agreement between Eq. (51) with stochastic simulation data is not good. It is in fact caused by the errors incurred in estimating $\rho_{k_{f}-1}$ by $N$, as shown in Fig. 24 by means of the relative deviation $d=\left(\rho_{k}-N\right) / N$ between for a few lattice sites behind the foremost particle.

In principle, for $W \neq 0$, it should be possible to perform an analysis in the spirit of Refs. [54,55] to get successively more accurate expressions for $\rho_{k_{f}-1}$, and correspondingly for the front speed and diffusion coefficient. In particular, such extensions might allow one to use the results in a wider parameter range, 


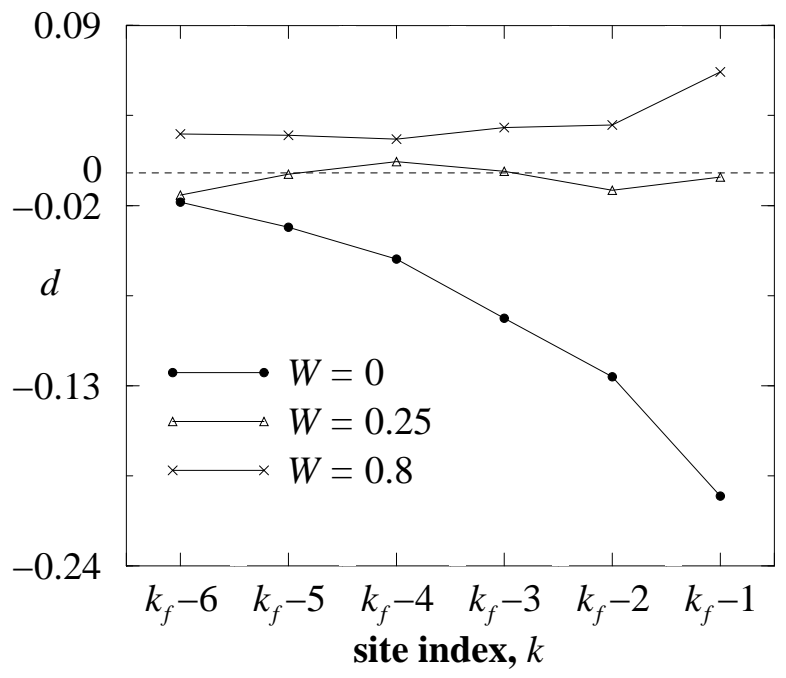

Fig. 24. Relative deviation $d=\left(\rho_{k}-N\right) / N$ of the average density from $N=\varepsilon /(\varepsilon+W)$ for the first six lattice sites to the left of the foremost occupied lattice site, $k_{f}$, for $D=\varepsilon=0.25$ and three different values of $W$.

such as $D / W \rightarrow \infty$ while $D / \varepsilon \sim \mathcal{O}(1)$, or $D / \varepsilon \rightarrow \infty$ while $W / \varepsilon \sim \mathcal{O}(1)$. However, inspection of the earlier analysis suggests that such higher order analytical expressions of $\rho_{k_{f}-1}$ are less trivial to obtain than one might expect at first sight. More precisely, for $W=0$, the master equation for the probability $p_{j}$ that the two foremost particles are separated by $j$ lattice sites can be closed in a simple manner $[54,55]$. For $W=0$ formalism, no particle gets annihilated, and as a result, the hierarchy of equations for the joint probability density distribution of the two foremost particles can be closed easily at the simplest level, since in the absence of annihilation, the third foremost particle never becomes the second foremost particle ("uncorrelated two-particle approach"). At this level, the expression of $\rho_{k_{f}-1}$ can then be analytically solved, leading to a better approximation than what we use in this paper for $W=0$. Of course, the master equation can be closed at a higher level, by considering more than two foremost particles to determine $\rho_{k_{f}-1}$, but then one does not obtain an analytical expression of $\rho_{k_{f}-1}$ ("correlated two-particle self-consistent approach"). As soon as $W \neq 0$, this is not true anymore: consider the following situation where the two foremost particles are next to each other. With annihilation of particles allowed, one of them can annihilate the other, and then the probability distribution function of the two foremost particles is crucially coupled to those which involve particles further back at the simplest level. It is therefore clear that for $W \neq 0$, the master equation for $p_{j}$ involves particles that are further back. While it is certainly possible to solve the master equation numerically, it does not appear to lead one to an analytical expression of $\rho_{k_{f}-1}$ that provides a better approximation than the one already used in Ref. [90]. 


\subsubsection{Fluctuating "Pulled" Fronts in Higher than One Spatial Dimensions}

Up until now in Sec. 2.6, we have only considered fronts on a one-dimensional lattice - and we have witnessed that with $N$ being the conditionally averaged number of particles per lattice site in the stable phase for propagating fronts into unstable states, the front propagates with a speed $v_{N}$, and simultaneously the front positions in different realizations of an ensemble undergoes diffusive wandering around its ensemble averaged position. In higher than one spatial dimensions, the situation gets more complicated, although the basics of front propagation in discrete particle and lattice systems remain unchanged - therein the interest is in the wandering properties of the front, not so much in its speed $[80,96,116]$.
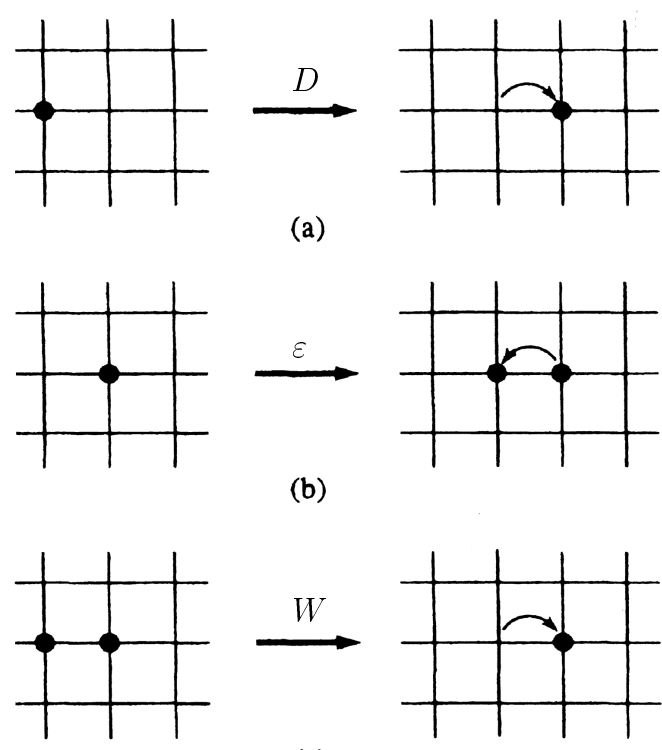

(a)

(b)
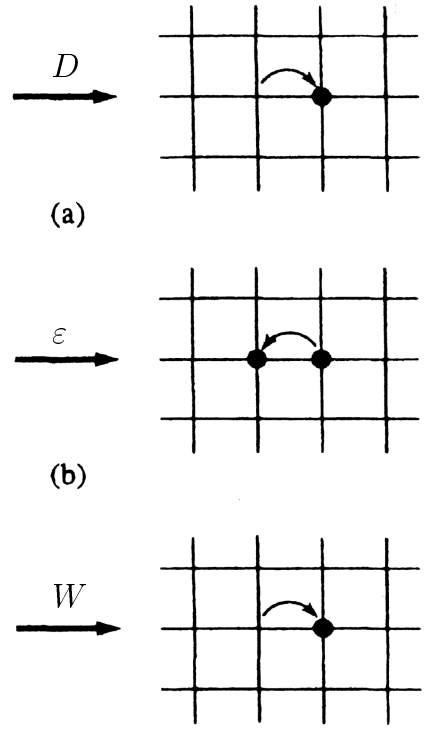

(c)

Fig. 25. Microscopic processes in the $d$-dimensional reaction-diffusion system $\mathrm{X}+\mathrm{X} \leftrightharpoons 2 \mathrm{X}$ along the $k_{l}$ direction $(l=1,2, \ldots, d)$ for a given value of $l$. On any lattice site, at most one $\mathrm{X}$ particle is allowed at any time. The dynamics along any one of the $k_{l}$ directions is exactly the same as that of Fig. 16.

Since all the studies on discrete particle and lattice system of propagating fronts in higher than one spatial dimensions have been on reaction-diffusion systems $\mathrm{X}+\mathrm{X} \leftrightharpoons 2 \mathrm{X}[80,96,116]$ or $\mathrm{X}+\mathrm{Y} \rightarrow 2 \mathrm{X}[72]$, to understand the general idea, it is best to first follow the reaction-diffusion system $\mathrm{X}+\mathrm{X} \leftrightharpoons 2 \mathrm{X}$ of Ref. [96] on a $d$ dimensional square lattice for various values of $d$ and briefly discuss its results. The system consists of a long lattice in the $k_{1}$ direction $\left(-L_{1} \leq\right.$ $\left.k_{1} \leq L_{1}\right)$ with a transverse length $L=L_{2}=L_{3}=\ldots=L_{d}$. Any lattice site can hold at most one $\mathrm{X}$ particle at any time. The microscopic dynamics of the particles along the $k_{l}$ direction $(l=1,2, \ldots, d)$ for a given value of $l$ is shown in Fig. 25 - the dynamics along any one of the $k_{l}$ directions is exactly the same as that of Fig. 16. With periodic boundary conditions applied along 
all the transverse directions $k_{2}, k_{3}, \ldots, k_{d}$ and the initial condition for the particle density $\phi_{\vec{k}}(t=0)=\Theta\left(k_{1}\right) N$, one studies front propagation in the $k_{1}$-direction. ${ }^{50}$

From this perspective, it is conceivable that the front properties can be studied by simply projecting the quantities defined in $d$ spatial dimensions on to the $k_{1}$ direction. Precisely this theme was developed in Ref. [96]: a mean particle density $\rho_{k_{1}}(t)$ was introduced by projecting the particle density profile in the $d$ dimensional space on to the $k_{1}$ direction as $\rho_{k_{1}}(t)=\left[\sum_{j=2}^{d} \phi_{\vec{k}}(t)\right] / L^{d-1} \cdot{ }^{51}$ One of the natural consequences of having to study the front properties by means of this projection is that one cannot define a front position based on the foremost particle as we have done so far in Secs. 2.6.2-2.6.5. To bypass this problem, a second quantity $K_{1}(t)=\left[\sum_{k_{1}=-L_{1}}^{L_{1}} \rho_{k_{1}}(t)\right] / N-L_{1}$ was introduced in Ref. [96] for the front position for any single realization. While the ensemble average $\left\langle K_{1}(t)\right\rangle \sim v_{N} t$ at long times, it was proposed that the wandering properties of the front around its ensemble averaged front position $\left\langle K_{1}(t)\right\rangle$ as a function of time can be measured by tracking the time development of quantity $w(t)$ [96], where [the angular brackets in Eq. (53) also denote ensemble averaging]

$$
w^{2}(t)=\left\langle\frac{2}{N} \sum_{k_{1}=-L_{1}}^{L_{1}} k_{1} \rho_{k_{1}}(t)-\left\langle K_{1}(t)\right\rangle^{2}-L_{1}^{2}\right\rangle .
$$

With these propositions, Ref. [96] invoked the scaling hypothesis for $w(t)$, i.e., $w(t) \sim t^{\alpha} F\left(t / L^{\beta}\right)$, and then continued further on to measure $\alpha$ and $\beta$ from simulations: for $d=2, \alpha=0.272 \pm 0.007$ and $\beta=1$; for $d=3$, the data are indistinguishable between the two possibilities $w(t) \sim t^{0.1}$ and $w(t) \sim \sqrt{\ln t}$; and for $d=4, \alpha=0$ (all simulations were carried out with $D=0.5$ and $\varepsilon / W=0.1){ }^{52}$

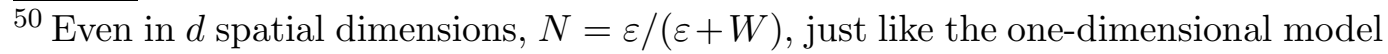
of Fig. 16. See footnotes 49 and 52.

${ }^{51}$ It is then clearly seen that $\rho_{k_{1}}(t)$ obeys the Fisher-Kolmogorov equation

$$
\begin{aligned}
& \frac{\partial \rho_{k_{1}}}{\partial t}=D\left[\rho_{k_{1}+1}+\rho_{k_{1}-1}-2 \rho_{k_{1}}\right] \\
& \quad+\left[\rho_{k_{1}+1}+2(d-1) \rho_{k_{1}}+\rho_{k_{1}-1}\right]\left[\varepsilon-(\varepsilon+W) \rho_{k_{1}}\right] .
\end{aligned}
$$

Notice that the corresponding one-dimensional problem in Sec. 2.6.5 also yields Eq. (52) with $d=1$ (although we never quite discussed it in Sec. 2.6.5). From Eq. (52), we find that $N=\varepsilon /(\varepsilon+W)$ for any $d$, just as we found in Eq. (32).

${ }^{52}$ At this juncture we briefly discuss the results of Ref. [72]. This paper considers front propagation in a three-dimensional reaction-diffusion system $\mathrm{X}+\mathrm{Y} \rightarrow 2 \mathrm{X}$, where initially the whole lattice is full of $\mathrm{Y}$ particles with an equilibrium concentration $N$, and at time $t=0$ one $\mathrm{X}$ particle is introduced at one location. Both the species diffuse with equal rate $D$ on the three-dimensional nearest neighbour 
It turns out however, that for the reaction-diffusion model $\mathrm{X}+\mathrm{X} \leftrightharpoons 2 \mathrm{X}$ of Fig. 25 , invoking the scaling relation to measure the front wandering is an extremely tricky issue. In fact, although $w(t)$ in Eq. (53) is a measure of the front wandering, the full measure of wandering of the $(d-1)$-dimensional interface for fronts in $d$ spatial dimensions is already lost when the projection $\rho_{k_{1}}(t)$ of $\phi_{\vec{k}}(t)$ along the $k_{1}$ direction is taken before the front wandering is defined. Truly speaking, if $\phi_{\vec{k}}(t)$ is not reduced to $\rho_{k_{1}}(t)$ before the scaling relation is invoked, then on a $d$-dimensional lattice, the problem of front wandering can be related to interfacial growth and associated scaling analyses of the interfacial roughness $[10,47,53,60,61]$. The scaling idea in these interfacial growth phenomena is the following: for interfacial growth in $d$ dimensions spanned by $\left(k_{1}, k_{2}, \ldots, k_{d}\right)$ co-ordinates [leading to a $(d-1)$-dimensional interface spanned by $\left(k_{2}, k_{3}, \ldots, k_{d}\right)$ co-ordinates], the "height" of the interface in the $k_{1}$ direction is described by $h_{k_{2}, k_{3}, \ldots, k_{d}}(t) \equiv h(t)$ at time $t$. With overline denoting the projection of a $d$-dimensional quantity on to one (along the $k_{1}$ direction) dimension [e.g., $\rho_{k_{1}}(t)=\overline{\phi_{\vec{k}(t)}}$ ], and the angular brackets denoting ensemble average, the actual scaling relation is given by $\mathcal{W}(t)=t^{\alpha} F\left(t / L^{\beta}\right)$, where $\mathcal{W}^{2}(t)=\overline{\left\langle[h(t)-\overline{h(t)}]^{2}\right\rangle}$. When the argument $\eta$ of $F(\eta)$ is $\ll 1$, then $F(\eta)$ behaves like a constant; on the other hand, for $\eta \gg 1, F(\eta) \sim \eta^{-\alpha}$. This implies that for $t \gg L^{\beta}, \mathcal{W}(t)$ saturates at $\mathcal{W}_{\text {sat }} \sim L^{\gamma}$, where $\gamma=\alpha \beta$.

Even with this modification, the actual values of $\alpha$ and $\beta$ in different dimensions for the $\mathrm{X}+\mathrm{X} \leftrightharpoons 2 \mathrm{X}$ reaction-diffusion model of Fig. 25 are not free from ambiguity $[80,116] .{ }^{53}$ The ambiguity stems from how $h(t)$ is related to $\phi_{\vec{k}}(t)$ [although it is clear that one should not use $\rho_{k_{1}}(t)$ for $h(t)$ to draw analogy between the front wandering properties and the interface growth roughness scaling relations on a $d$-dimensional lattice]. To illustrate this point, let us consider the example case for $d=2$. It turns out that when $h(t)$ equals a simple local coarse-graining over the field values $\phi_{\vec{k}}(t)$ within a cubic box of size $(2 \ell+1)^{d}$ around $\vec{k}$, then for $\ell=1$, one obtains $\alpha=0.29 \pm 0.01$ and $\gamma \simeq 0.4 \pm 0.02[116](\alpha=0.27 \pm 0.01$ and $\gamma \simeq 0.41 \pm 0.02$ [80]). Interestingly enough, these exponents are surprisingly close to the KPZ exponents for a

lattice sites, and any $\mathrm{Y}$ particle coming in contact with any other $\mathrm{X}$ particle on the same lattice site is instantaneously and irreversibly converted to an X particle this is the $d$-dimensional generalization of the one-dimensional lattice model of Sec. 2.6.2. The object of study was to see the deviations of $v_{N}$ from the prediction of the Fisher-Kolmogorov equation, $v^{*}$. The numerical finding was that $v_{N}$ deviates from $v^{*}$. Not only that there was no study of the broadening of the front region by means of measuring $w(t)$ therein, but also the front speed as a function of the total average particle density was not carefully fitted with any theoretical curve. In this context, we refer to Fig. 2 of Ref. [80], which shows, for the reaction-diffusion model $\mathrm{X}+\mathrm{X} \leftrightharpoons 2 \mathrm{X}$ of Fig. 25, that for $\varepsilon / D \rightarrow 0$, front speed $v_{N}$ in $d>1$ does behave as $\sqrt{\varepsilon}$ as the Fisher-Kolmogorov equation would predict.

${ }^{53}$ We note here that only $W=D$ case was considered in Ref. [80]. 
two-dimensional interface [116] $(\alpha=0.245 \pm 0.003, \gamma=0.393 \pm 0.003$ [77]). However, it has been shown that with increasing values of $\ell$, i.e., $\ell=2,3,4$ etc., the exponents $\alpha$ and $\gamma$ approach the KPZ exponents for a one-dimensional interface $(\alpha=1 / 3, \gamma=0.5)$ [80]. So the general picture that emerges from these analyses is that as one looks at progressively longer length scales, the wandering properties of the front makes a transition from a non-KPZ to a KPZ behaviour [80].

With $\ell=4$, the KPZ scaling behaviour for the front wandering on a three $(\gamma=0.393[77])$ and a four dimensional lattice $(\gamma=0.313[77])$ for the $\mathrm{X}+\mathrm{X} \leftrightharpoons$ $2 \mathrm{X}$ reaction-diffusion model of Fig. 25 has been recovered [80]. These results together indicate that in this model, any generic upper critical dimension has to be higher than 4. More simulations are however needed to conclusively clarify this point.

\subsection{Convergence of the Asymptotic Front Speed to $v^{\dagger}$ for Fluctuating Pushed Fronts as $N \rightarrow \infty$}

We had earlier pointed out that at the time of writing this review article, I have not seen any work on how $v_{N}$ behaves as a function of $N$ in the limit $N \rightarrow \infty$. Nevertheless, in Sec. 2.7, we will show that if one incorporated the discrete nature of the particles by means of an effective cutoff ${ }^{54}$ following the ideas of Brunet and Derrida as described in Sec. 2.2 [19], then the corresponding front speed $v_{N}$ must behave as $v^{\dagger}-v_{N} \sim N^{-\gamma}$, where $\gamma$ is a positive number (c.f. footnote 8).

The route we follow to demonstrate this power law convergence of $v_{N}$ to $v^{\dagger}$ is by means of considering an example of a front propagation from a stable state to a (meta)stable state in [56]

\footnotetext{
${ }^{54}$ The introduction of an effective cutoff to model the discreteness of particles, even for fluctuating pushed fronts, is not unusual. Consider for example the following deterministic equation in continuous space and time $[12,100]$

$$
\frac{\partial \phi}{\partial t}=D \frac{\partial^{2} \phi}{\partial x^{2}}+\phi+\left(b^{-1}-1\right) \phi^{2}-b^{-1} \phi^{3}
$$

for $b>0$. This system then has a linearly unstable state at $\phi=0$, and a stable state at $\phi=1$. In a discrete particle and lattice model of this model with $\phi_{k}=\left\langle\left\langle N_{k}\right\rangle\right\rangle / N$, where $N$ is the (conditionally) average number of particles per lattice site at the stable phase of the front, at the tip, the value of $\phi$ is once again of $\mathcal{O}(1 / N)$. The effect of discreteness of particles on the front, once again, can then be mimicked by putting a growth cutoff for $\phi$ at $\varepsilon \simeq 1 / N$.
} 


$$
\frac{\partial \phi}{\partial t}=D \frac{\partial^{2} \phi}{\partial x^{2}}+\left(1-\phi^{2}\right)(\phi+a),
$$

where $0<a<1$. This equation, which appears with the name of GinzburgLandau equation or with the so-called Schlögl model, has two stable states, $\phi= \pm 1$, and an unstable state at $\phi=-a$. For a steep initial condition that connects $\phi=1$ to $\phi=-1$, a front propagates from the $\phi=1$ to the $\phi=-1$ state with speed $v^{\dagger}=a \sqrt{2}$; and the corresponding front profile in terms of $\zeta=x-v^{\dagger} t$ is described by [56]

$$
\phi^{\dagger}(\zeta)=-\tanh \left[\lambda^{\dagger}\left(\zeta-\zeta_{0}\right) / 2\right]
$$

such that $\lambda^{\dagger}=\left[v^{\dagger}+\sqrt{v^{\dagger 2}+8 D(1-a)}\right] /(2 D) .{ }^{55}$ Notice that the Ginzburglandau equation (55) does not fall in the category of fronts propagating into unstable state, the procedure to obtain $v^{\dagger}-v_{\varepsilon} \sim|\varepsilon|^{-\gamma}$ in Eq. (55) is very instructive, and it can be easily applied to pushed fronts propagating into unstable states. ${ }^{56}$

As we add a growth cutoff in the Ginzburg-Landau equation (55) at $\phi=-1+\varepsilon$, we can use the technique developed by Brunet and Derrida (c.f. Sec. 2.2 [19]) to solve for the asymptotic front speed $v_{\varepsilon}$ [56]. Let us denote the location, in the comoving co-ordinate $\zeta=x-v_{\varepsilon} t$, where the value of $\phi$ reaches $-1+\varepsilon$ by $\zeta_{0}$. For $\varepsilon \rightarrow 0$, Eq. (55) can clearly be linearized on the right of $\zeta_{0}$. In addition, in the left neighbourhood of $\zeta_{0}$, the value of $\phi$ is still infinitesimally close to -1 , which allows one to linearize Eq. (55) around the unstable state once again. This yields the following form of the front solution [56] ${ }^{57}$

\footnotetext{
$\overline{55}$ It is not surprising at all that the front solutions in Eqs. (54) and (55) bear strong similarities [29] — with appropriate redefinition of $\phi$ and rescaling of the diffusion coefficient $D$ and time $t$, one equation can be converted into another. However, one has to be careful here: despite the convertibility of one form to another, the difference between the nature of the fronts in Eqs. (54) and (55) stems from the fact that $b>0$ in Eq. (55) corresponds to $a>1$ in Eq. (54), for which the front propagation is into a linearly unstable state. On the other hand, $0<a<1$ in Eq. (54) corresponds to $b<0$ in Eq. (55) - in this case, front propagates from a stable to an (meta)stable state.

56 Notice that with $v^{\dagger}=a \sqrt{2}$, the front profile (56) is directly obtained by solving Eq. (55). In general, in the comoving frame for any given asymptotic front speed $v_{\text {as }}$, upon linearizing Eq. (55) around $\phi=-1$ and using the front profile ansatz $\phi(\zeta) \sim-1+A e^{-\lambda \zeta}$ for $\zeta \rightarrow \infty$, two roots of the exponent $\lambda$ are obtained: $\lambda_{1}=$ $\left[v_{\mathrm{as}}+\sqrt{v_{\mathrm{as}}^{2}+8 D(1-a)}\right] /(2 D)>0$ and $\lambda_{2}=\left[v_{\mathrm{as}}-\sqrt{v_{\mathrm{as}}^{2}+8 D(1-a)}\right] /(2 D)<0$. In the corresponding front solution $\phi(\zeta) \sim-1+A_{1} e^{-\lambda_{1} \zeta}+A_{2} e^{-\lambda_{2} \zeta}, A_{2}$ must be zero for the solution to be physically relevant. This condition is clearly obeyed by the front profile (56).

57 The reason for expressing the coefficient of $e^{-\lambda_{2} \zeta}$ in Eq. (57) in this manner is
} 


$$
\begin{aligned}
\phi^{(0)}(\zeta) & =-1+A_{1} e^{-\lambda_{1}^{\dagger} \zeta}+A_{2}\left(v^{\dagger}-v_{\varepsilon}\right) e^{-\lambda_{2}^{\dagger} \zeta} & & \text { for large } \zeta, \text { but } \zeta<\zeta_{0} \\
& =-1+\varepsilon e^{-v_{\varepsilon}\left(\zeta-\zeta_{0}\right)} & & \text { for } \zeta \geq \zeta_{0},
\end{aligned}
$$

where the expressions of $\lambda_{1}^{\dagger}$ and $\lambda_{2}^{\dagger}$ are obtained from footnote 56 after having $v_{\text {as }}$ substituted by $v^{\dagger}$. As the two solutions of Eq. (57) and their derivatives are matched at $\zeta_{0}$ (with the condition that $A_{1}$ and $A_{2}$ are independent of $\varepsilon$; see footnote 57 ), it is easily seen that $\zeta_{0} \sim|\ln \varepsilon|$ and $[56]^{58}$

$$
v^{\dagger}-v_{\varepsilon} \sim \varepsilon^{1-\lambda_{2}^{\dagger} / \lambda_{1}^{\dagger}}
$$

For $D=a=1 / 2, \lambda_{2}^{\dagger} / \lambda_{1}^{\dagger}=-1 / 2$, and $v^{\dagger}-v_{\varepsilon} \sim \varepsilon^{3 / 2}$. This scaling of $v^{\dagger}-v_{\varepsilon}$ has been observed in the simulation [56], and it is shown in Fig. 26.

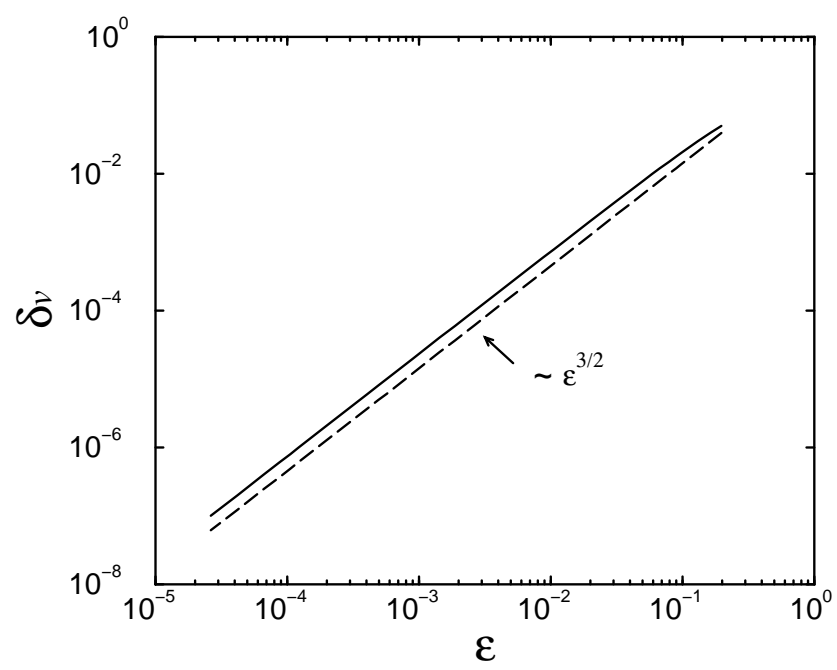

Fig. 26. The log-log curve of $\delta v=v^{\dagger}-v_{\varepsilon}$ vs. $\varepsilon$ for the Ginzburg-Landau equation (55) with $D=a=1 / 2$ [56]. Solid line: simulation data, dashed line: visual aid for the $\delta v \sim \varepsilon^{3 / 2}$ behaviour predicted in Eq. (58).

As already pointed out, the exercise can be repeated to obtain a power law behaviour of $v^{\dagger}-v_{\varepsilon}$ for the Eq. (54), where the front does propagate into an unstable state. Based on Brunet and Derrida's insight that the effect of

actually quite subtle. The idea lies in the fact that just like we linearize Eq. (55) with a growth cutoff around -1 for large $\zeta$ to obtain Eq. (57), we can also linearize it around 1 for $\zeta \rightarrow-\infty$. This linearization suggests an equivalent form of the front solution $\phi^{(0)}(\zeta)=1-B_{1} e^{\lambda_{1^{\prime}}^{\dagger} \zeta}-B_{2}\left(v^{\dagger}-v_{\varepsilon}\right) e^{\lambda_{2^{\prime}}^{\dagger} \zeta}$ for $\zeta \rightarrow-\infty$, where $\lambda_{1^{\prime}}^{\dagger}=$ $\left[-v^{\dagger}+\sqrt{v^{\dagger 2}+8 D(1+a)}\right] /(2 D)>0$ and $\lambda_{2^{\prime}}^{\dagger}=\left[-v^{\dagger}-\sqrt{v^{\dagger 2}+8 D(1+a)}\right] /(2 D)<0$. The linearized equations at $\zeta \rightarrow \pm \infty$ involve $v_{\varepsilon}$ as an input parameter. However, in this form of $\phi^{(0)}(\zeta)$ at $\zeta \rightarrow \pm \infty, A_{1}, A_{2}, B_{1^{\prime}}$ and $B_{2^{\prime}}$ all become independent of $\varepsilon$. ${ }^{58}$ Equation (58) has also been obtained by Chomaz et al [25], in a study of the influence of a fixed boundary in a system with a convective instability. 
discreteness in terms of particles and the lattice is well captured by the choice $\varepsilon \sim 1 / N$ as $N \rightarrow \infty$, the above analysis indicates that in a discrete particle and lattice model of fluctuating pushed fronts $v^{\dagger}-v_{N}$ would indeed behave as $N^{-\gamma}$ for some $\gamma>0$. However, at the time of writing this review article, I have not seen any empirical verification of this prediction at this point.

\subsection{Epilogue I}

As we discussed front propagation in discrete particle and (mostly one-dimensional) lattice systems throughout Sec. 2, we started with an asymptotically large value of $N$ in Sec. 2.2, and from there onwards we gradually reduced the value of $N$ till Sec. 2.6. We have witnessed that pulled fronts in discrete particle and lattice systems do not exist, at best they are weakly pushed. With decreasing value of $N$, the weakly pushed nature of these fronts becomes more and more prominent, and the front properties start to depend heavily on the details of the models under consideration. Because of these complications at intermediate values of $N$, in general, it is very difficult to obtain a firstprinciple based predictive expression of $v_{N}$ for fluctuating "pulled" fronts. In a contradictory trend to this, however, at the limit of $N$ when there is at most one particle allowed per lattice site, front speed and the front diffusion coefficient become tractable again (c.f. Sec. 2.6.5), giving rise to exact relation like Eq. (46), or approximate theoretical expressions like Eqs. (46) and (51).

Even if we exclude the small or moderately large (but not asymptotically large!) values of $N$ from these concluding remarks on Sec. 2, so many different features of discrete particle and lattice systems of fronts propagating into unstable states for $N \rightarrow \infty$ discussed in Secs. 2.2, 2.4-2.5 and 2.7 may at first seem a bit puzzling to the reader. A moment's reflection, however, shows that the origin of such versatile phenomena can be intuitively understood in a unified perspective - to be more precise, from the stability spectrum of (deterministic) pulled and pushed fronts propagating into unstable states, already briefly discussed at the end of Sec. 1.1: the gapless stability spectrum of pulled fronts leads to its power law convergence (4) of the front speed $v(t)$ to $v^{*}$ in time for the asymptotic front speed $[38,101,102]$ has already been noted in Sec. 1.1. On the other hand, stability spectrum of pushed fronts is gapped $[22,23,37,56,92,101,102]$, which leads to exponential convergence of the front speed $v(t)$ to $v^{\dagger}[56,102]$.

What we observe in fluctuating "pulled" fronts and as well as in fluctuating pushed fronts for $N \rightarrow \infty$ is intimately connected to their stability spectra. For pulled fronts, the gapless nature of the stability spectrum is indeed another representation of the linear marginal stability criterion. It therefore must come as no surprise that any change in the front dynamics close to the linearly 
unstable state must affect the front properties severely. In Secs. 2.2 and 2.5 this is manifested by the very slow convergence of $v_{N}$ and $D_{f}$ to $v^{*}$ and zero respectively, while the stability spectrum of fluctuating "pulled" fronts itself becomes gapped with a very small gap $\propto 1 / \ln ^{2} N$ (c.f. Sec. 2.4). On the other hand, for pushed fronts, the spectrum is gapped, and any change in the front dynamics close to the linearly unstable [or (meta)stable] state is expected to affect the front properties minimally. This is then reflected in the stronger power law (in $N$ ) convergence of $v_{N}$ to $v^{\dagger} .{ }^{59}$

We finally close Sec. 2 on discrete particle and lattice systems of fluctuating fronts propagating into unstable states by pointing out a few problems, whose solutions are not yet completely settled. These are: (a) for fluctuating "pulled" fronts a first principle based predictive theory for the front speed $v_{N}$ at intermediate values of $N$, (b) for the model of Sec. 2.6.3, why one observes mean-field Fisher-type behaviour even at a reasonably small value of $N$, (c) a better analytical prediction for the model of Sec. 2.6.5 for a larger set of parameter values, and (d) possible existence of an upper critical dimension for fluctuating "pulled" fronts, when there are at most one particle allowed per lattice site (c.f. Sec. 2.6.6).

\section{Field-theory of Fluctuating Fronts: External Fluctuations}

\subsection{External Fluctuations, Multiplicative Noise and Novikov's Theorem}

We have seen earlier in Sec. 1.1 how propagating fronts have been observed in many systems related to physics, chemistry and biology. In all of these cases, the evolution equations of the propagating fronts invariably involve some parameters that relate to experimental situations, such as parameters in chemical or biological reaction kinetics, an externally imposed fields etc. When these parameters correspond to externally imposed fields, then the variation in these fields acts as a varying external influence on the system — such as an externally imposed electric or magnetic field, or light intensity in a photosensitive reaction. Similarly, when these parameters originate within the system itself, then usually they are law-of-mass-action-based mesoscopic mean-field theory estimates of quantities that originate in the underlying microscopic dynamics. For the most part, (Langevin-type) field-theoretical studies on the effects of fluctuations in systems with propagating fronts have been motivated by these phenomenological considerations. Other significant motivating factors for the field-theoretical approaches have been phase transitions induced by

\footnotetext{
$\overline{{ }^{59} \text { Let }}$ us just state here that the theoretical arguments indicate that the diffusion coefficient of fluctuating pushed fronts made of discrete particles on a lattice should scale as $1 / N$; however, for now we will leave this issue until Sec. 4.2.1.
} 
noise $[11,18,44,93]$, and the discovery that surface roughness in various growth processes fall under a handful number of universality classes $[10,40,53,60,61]$.

In my opinion, for systems admitting propagating front solutions, external fluctuations are easier to handle than the internal ones - not only will we find ample evidences of this later on, but also two simple examples at this stage will make this point clear. Consider a front dynamics under the influence of an external electric field $\vec{E}=E \hat{x}$ that couples linearly to the front field $\phi$, also propagating in the $x$-direction. Fluctuations in the electric field of magnitude $\delta E(x, t)$ around its mean value $E^{(0)}$ simply gives rise to a term $\propto \delta E(x, t) \phi(x, t)$ in the evolution equation of the front. Contrast this situation with a chemical reaction front: therein the deterministic front evolution equation is already a mesoscopic (mean-field) description of the underlying microscopic process - these mesoscopic descriptions collectively give rise to the very definition of the front field $\phi$, and as well as to the mass-action based parameters in the dynamics such as the reaction rate. When fluctuations are to be considered in such systems, then in general, fluctuations in both the number of particles per correlation volume (which usually defines the front field) and as well as in the parameters are to be considered together. In the full description of these fluctuations, naturally, complicated correlations between the fluctuating front field and the parameters are unavoidable. To get a reasonable grip on the theoretical handling of these processes, the mass-action based mesoscopic parameters for the internal dynamics are often kept intact in the dynamics of the system (we have seen a large number of examples of it in Sec. 2) and separate fluctuation terms are added by hand to the evolution equations to mimic the internal fluctuations.

Precisely because of these subtleties, field theories for the internal fluctuations often prove to be very tricky. For now, we will leave it for Sec. 4. As for Sec. 3 , we will devote it to the field-theory of external fluctuations on propagating fronts.

To begin with, let us consider the deterministic equation for a scalar front in one spatial dimension coupled to $n$ external fields $a_{i}, i=1,2, \ldots, n$ :

$$
\frac{\partial \phi}{\partial t}=\mathcal{F}\left(\left\{a_{i}\right\}, \phi\right)
$$

The effect of the fluctuations in the fields $a_{i}$ around their mean-field values $a_{i}^{(0)}$ on the front properties is then given by

$$
\frac{\partial \phi}{\partial t}=\mathcal{F}\left(\left\{a_{i}^{(0)}\right\}, \phi\right)+\left.\sum_{i=1}^{n} \sum_{k} \frac{1}{k !} \frac{\delta^{k} \mathcal{F}}{\delta a_{i}^{k}}\right|_{a_{i}^{(0)}}\left[a_{i}(x, t)-a_{i}^{(0)}\right]^{k}
$$


In Eq. (60), the functional derivative $\left.\frac{\delta^{k} \mathcal{F}}{\delta a_{i}^{k}}\right|_{a_{i}^{(0)}}$ is a function of the front field. What is the precise form of this function depends on how the fields are coupled to the front dynamics, but the fact remains that in the evolution equation of the front, the coefficients of the fluctuations in the external fields are multiplied by functions of the front field - Eq. (60) is thus an equation with multiplicative noise.

Before we proceed further, it is however necessary to first properly interpret the multiplicative noise term in Eq. (60). This brings us to the well-documented Itô vs. Stratonovich dilemma for multiplicative noise $[45,52]$. In this review article, we will not delve deep into the details of which interpretation is the right one, but it is extremely important to be clear about the fact that throughout Sec. 3, the noise term in Eq. (60) will be interpreted in the Stratonovich sense. Section 3, in fact, is a collection of works done on the field theory of external fluctuations on propagating fronts for the last two decades, ${ }^{60}$ and all these works interpreted the noise term in the Stratonovich sense.

We will now see in Sec. 3.1.1 that in the context of front propagation, the so-called Novikov's theorem [85] has proved to be a very useful tool for theoretical analysis with Stratonovich interpretation of multiplicative noise. It should however be kept in mind that under general circumstances, Novikov's theorem is not an automatic gateway to success in these problems. First of all, the theorem can handle noise only with Gaussian statistics. Secondly, it is practically useful only if the spatial and temporal part of the noise factorize, and in the limit when both the noise correlation length and correlation time go to zero.

\subsubsection{Novikov's Theorem in the Context of Front Propagation in Reaction- Diffusion Systems}

Consider the stochastic differential equation describing a general reactiondiffusion process coupled to a single external field ${ }^{61}$

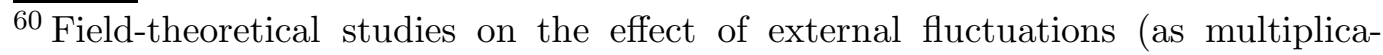
tive noise) on propagating front in the context of reaction-diffusion systems date back to the early 1980s $[79,107]$. Some threads of it were picked up in the early 1990s [94,108]; but all of these works considered only weak noise, i.e., the case of $\left|\left[a_{i}-a_{i}^{(0)}\right] / a_{i}^{(0)}\right| \ll 1$ in Eq. (60). The late 1990s till now witnessed a surge of activities in this field $[3,4,97-99,105,106]$ - they considered multiplicative noise that is not necessarily weak.

${ }^{61}$ Novikov's theorem can be easily generalized to arbitrary spatial dimensions and as well as to the case when the front field is a vector field, see Ref. [99]. Generalizations to more than one external field coupled to the front dynamics is also trivial.
} 


$$
\frac{\partial \phi}{\partial t}=D \frac{\partial^{2} \phi}{\partial x^{2}}+f(\phi)+\tilde{\varepsilon}^{1 / 2} g(\phi) \eta(x, t),
$$

where $\tilde{\varepsilon} \eta(x, t)$ is the space-time dependent fluctuation in the external field, $\tilde{\varepsilon}$ is the strength of the (multiplicative) noise, and $\eta(x, t)$ has zero mean with a correlation time $\tau_{c}$ and correlation length $\lambda_{c}$. The factorized temporal and spatial correlations are expressed by the functions $C_{t}$ and $C_{x}$ respectively, such that (the angular brackets with a subscript $\eta$ denote averaging over the noise)

$$
\begin{gathered}
\left\langle\eta(x, t) \eta\left(x^{\prime}, t^{\prime}\right)\right\rangle_{\eta}=2 C_{t}\left(\left|t-t^{\prime}\right| ; \tau_{c}\right) C_{x}\left(\left|x-x^{\prime}\right| ; \lambda_{c}\right) \quad \text { and } \\
\int d x^{\prime} C_{x}\left(\left|x-x^{\prime}\right| ; \lambda_{c}\right)=\int d t^{\prime} C_{t}\left(\left|t-t^{\prime}\right| ; \tau_{c}\right)=1
\end{gathered}
$$

The difficulty with using Eq. (61) directly is that although the mean of $\eta(x, t)$ is zero, in the Stratonovich interpretation, the stochastic term in Eq. (61) $[\propto g(\phi) \eta(x, t)]$ does not have zero mean. This is precisely where Novikov's theorem comes in - its purpose is to (non-perturbatively) rewrite Eq. (61) in such a manner that the fluctuating term in it does have zero mean. Novikov's theorem, however, is applicable only if $\eta(x, t)$ itself is a Gaussian random function with zero mean [85].

The exact statement for Novikov's theorem is the following: Suppose $h(s)$ is a Gaussian random function with zero mean value and correlation

$$
\left\langle h(s) h\left(s^{\prime}\right)\right\rangle_{h}=\mathcal{H}\left(s, s^{\prime}\right)
$$

then for any functional $H(\{h\})$, the following result holds [85]:

$$
\langle h(s) H(\{h\})\rangle_{h}=\int_{-\infty}^{\infty} d s^{\prime} \mathcal{H}\left(s, s^{\prime}\right)\left\langle\frac{\delta H(\{h\})}{\delta h\left(s^{\prime}\right)}\right\rangle_{h} .
$$

Equation (64) does not involve any approximations. However, to make any practical use of it for any analytical calculation in the present context, Novikov's theorem is useful only if the correlation time $\tau_{c}$ and correlation length $\lambda_{c}$ become much smaller than the respective time and length scales of the propagating front ${ }^{62}$ - for example when $\tau_{c}$ is much smaller than the inverse reaction rate and $\lambda_{c}$ is much smaller than the front width. In these cases, one can use both $\tau_{c}$ and $\lambda_{c} \rightarrow 0$ and reduce Eq. (62) to

$$
\left\langle\eta(x, t) \eta\left(x^{\prime}, t^{\prime}\right)\right\rangle_{\eta}=2 \delta\left(t-t^{\prime}\right) \delta\left(x-x^{\prime}\right) .
$$

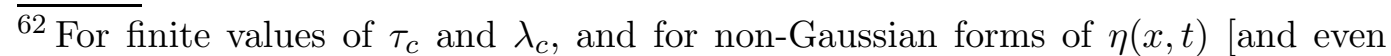
for non-factorizable spatiotemporal correlations, unlike Eq. (62)], it is possible to formulate a Novikov-type theorem by carrying out a cumulant expansion of the noise. See Ref. [106]. 
The limit of both $\tau_{c}$ and $\lambda_{c} \rightarrow 0$, however, causes certain intricacies in the application of Novikov's theorem, as we now illustrate below. From Eqs. (6365) we have

$$
\begin{aligned}
\langle g[\phi(x, t)] \eta(x, t)\rangle_{\eta}=2 \int_{-\infty}^{\infty} d t^{\prime} \int_{-\infty}^{\infty} d x^{\prime} & C_{t}\left(\left|t-t^{\prime}\right| ; \tau_{c}\right) C_{x}\left(\left|x-x^{\prime}\right| ; \lambda_{c}\right) \times \\
& \times\left\langle g^{\prime}[\phi(x, t)] \frac{\delta \phi(x, t)}{\delta \eta\left(x^{\prime}, t^{\prime}\right)}\right\rangle_{\eta}
\end{aligned}
$$

After having solved $\phi(x, t)$ from Eq. (61) as

$$
\begin{gathered}
\phi(x, t)=\int_{-\infty}^{\infty} d t^{\prime \prime}\left[D \frac{\partial^{2} \phi\left(x, t^{\prime \prime}\right)}{\partial x^{2}}+f\left[\phi\left(x, t^{\prime \prime}\right)\right]+\tilde{\varepsilon}^{1 / 2} g\left[\phi\left(x, t^{\prime \prime}\right)\right] \eta\left(x, t^{\prime \prime}\right)\right] \times \\
\times \Theta\left(t-t^{\prime \prime}\right)
\end{gathered}
$$

the expression $\frac{\delta \phi(x, t)}{\delta \eta\left(x^{\prime}, t^{\prime}\right)}$ in Eq. (66) reads

$$
\frac{\delta \phi(x, t)}{\delta \eta\left(x^{\prime}, t^{\prime}\right)}=\tilde{\varepsilon}^{1 / 2} g\left[\phi\left(x, t^{\prime}\right)\right] \Theta\left(t-t^{\prime}\right) \delta\left(x-x^{\prime}\right)
$$

which subsequently implies that

$$
\begin{aligned}
\langle g[\phi(x, t)] \eta(x, t)\rangle_{\eta}=2 \tilde{\varepsilon}^{1 / 2} C_{x}(0 & \left.; \lambda_{c}\right) \int_{-\infty}^{\infty} d t^{\prime} C_{t}\left(\left|t-t^{\prime}\right| ; \tau_{c}\right) \times \\
& \times\left\langle g^{\prime}[\phi(x, t)] g\left[\phi\left(x, t^{\prime}\right)\right]\right\rangle_{\eta} \Theta\left(t-t^{\prime}\right) .
\end{aligned}
$$

One can now clearly see that the replacement of $C_{t}\left(\left|t-t^{\prime}\right| ; \tau_{c}\right)$ by $\delta\left(t-t^{\prime}\right)$ in the limit of $\tau_{c} \rightarrow 0$ results in a perfectly well behaved r.h.s. of Eq. (69), but the spatial white noise limit in the case of $\lambda_{c} \rightarrow 0$ does not, since the $\lambda_{c} \rightarrow 0$ limit sends $C_{x}\left(0 ; \lambda_{c}\right)$ to $\infty$. In most papers on field-theory of front propagation with multiplicative (external) noise, the limit $\tau_{c} \rightarrow 0$ in Eq. (62) is often taken implicitly, while the explicit dependence on $C_{x}\left(x-x^{\prime} ; \lambda_{c}\right)$ is left intact. In this limit, Eq. (69) reduces to

$$
\tilde{\varepsilon}^{1 / 2}\langle g[\phi(x, t)] \eta(x, t)\rangle_{\eta}=\varepsilon\left\langle g^{\prime}[\phi(x, t)] g[\phi(x, t)]\right\rangle,
$$

where $\varepsilon=\tilde{\varepsilon} C_{x}\left(0 ; \lambda_{c}\right)$ and $\Theta(0)=1 / 2$.

In order to avoid the difficulty associated with the remaining $C_{x}\left(0 ; \lambda_{c}\right)$ in Eq. (70) approaching infinity in the limit of $\tau_{c}$ and $\lambda_{c} \rightarrow 0$, operationally (i.e., in a 
computer simulation) one simply discretizes both space and time in units of $\Delta x$ and $\Delta t$. These discretization units then act as natural ultraviolet cutoffs. So long as $\Delta t$ is sufficiently small, the results are independent of $\Delta t$. On the other hand, when $\Delta x$ is sufficiently small, the Gaussian representation $C_{x}\left(0 ; \lambda_{c}\right)$ of $\delta$-function in space is replaced by a square representation of $\delta$-function, such that $\lambda_{c}=\Delta x$, and one uses $[3,97]$

$$
C_{x}\left(0 ; \lambda_{c} \rightarrow 0\right)=\frac{1}{\Delta x}
$$

That this is the right interpretation is confirmed by very good agreements between the theoretical and the simulation results. ${ }^{63}$

With Eq. (70), we can finally rewrite (61) as (see for example Ref. [3])

$$
\frac{\partial \phi}{\partial t}=D \frac{\partial^{2} \phi}{\partial x^{2}}+f_{g}(\phi)+\tilde{\varepsilon}^{1 / 2} R(\phi, x, t)
$$

where $f_{g}(\phi)=f(\phi)+\varepsilon g(\phi) g^{\prime}(\phi)$. Notice now that

$$
R(\phi, x, t)=g(\phi) \eta(x, t)-\tilde{\varepsilon}^{1 / 2} C_{x}\left(0 ; \lambda_{c}\right) g(\phi) g^{\prime}(\phi)
$$

has zero mean and the correlation property that ${ }^{64}$

$$
\left\langle R(\phi, x, t) R\left(\phi, x^{\prime}, t^{\prime}\right)\right\rangle_{\eta}=\left\langle g[\phi(x, t)] \eta(x, t) g\left[\phi\left(x^{\prime}, t^{\prime}\right)\right] \eta\left(x^{\prime}, t^{\prime}\right)\right\rangle_{\eta}+O\left(\tilde{\varepsilon}^{1 / 2}\right) .
$$

\footnotetext{
${ }^{63}$ Equations (66-69) are indeed a fancy way of deriving Eq. (70). Because these equations use Novikov's theorem, they are only applicable if $\eta$ is a Gaussian random variable. When $\eta$ is not Gaussian, then there is a simpler way to derive Eq. (70) for the Stratonovich interpretation of Eq. (61): see for example Ref. [45] on the issue of the equivalence of Itô and Stratonovich integrals.

${ }^{64}$ We will see in Sec. 3.1.2 that the front speed is obtained from the equation $\frac{\partial \phi}{\partial t}=D \frac{\partial^{2} \phi}{\partial x^{2}}+f_{g}(\phi)$, and the right parameter for the effect of the stochastic term on the front speed is $\varepsilon$ and not $\tilde{\varepsilon}$. In that sense, not only is it clear that the choice of $\Delta x$ does affect the front speed, but also in the limit $\Delta x \rightarrow 0$, Eq. (72) captures the entire effect of the external fluctuations on the front speed and not just up to some order in a perturbation expansion in powers of $\tilde{\varepsilon}$. The diffusion coefficient of the so-called Goldstone mode $D_{G}[94,109,110]$, however, involves the assumption that $\tilde{\varepsilon} \ll 1$, so that one can neglect the $O\left(\tilde{\varepsilon}^{1 / 2}\right)$ term in Eq. (74). The issue of $D_{G}$ is however a bit more complicated - although the $\tilde{\varepsilon}$-dependence of the diffusion coefficient of the Goldstone mode is more subtle than a simple series expansion in powers of $\tilde{\varepsilon}$, the fact remains that for $\tilde{\varepsilon} \ll 1$, the contribution of the $O\left(\tilde{\varepsilon}^{1 / 2}\right)$ term in Eq. (74) indeed yields a higher order correction [3].
} 


\subsubsection{Reaction-Diffusion Systems: The Effect of Multiplicative Noise on the Front Speed and the Diffusion Coefficient of the So-called Goldstone Mode}

From the discussion in Sec. 3.1.1, it is clear that in the context of front propagation, application of Novikov's theorem to rewrite Eq. (61) to (72) is not a series expansion in powers of $\tilde{\varepsilon}$. Neither does Eq. (72) involve any approximations. Instead, it is simply a way to separate the systematic part and the fluctuating part of the stochastic differential equation (61) - as we will now see, rewriting Eq. (61) in the form of Eq. (72) has the advantage that the systematic part gives rise to a steady shift in the front speed, while the fluctuating part gives rise to two other phenomena at two different time scales: the (random) displacement of the front from its uniformly translating position at long time scales, and shape fluctuation of the front around its instantaneous position at short time scales (see the illustration in Fig. 27). A very simple and instructive illustration of this appears in Refs. [79,94,97, 107, 108], and here we will follow it to make our point.

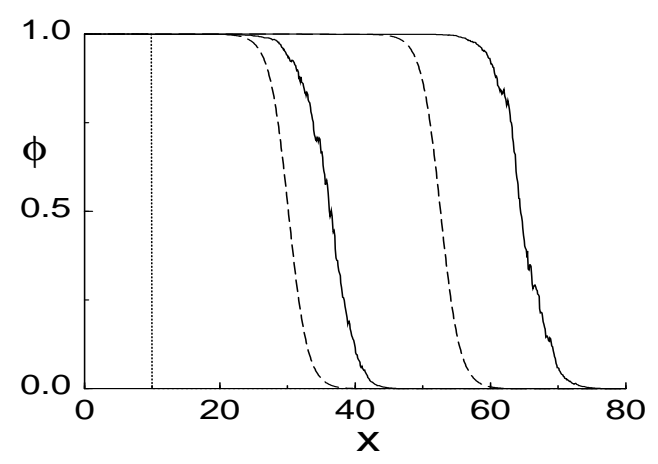

Fig. 27. Snapshots of fronts (with multiplicative noise) in the stochastic differential equation $\frac{\partial \phi}{\partial t}=\frac{\partial^{2} \phi}{\partial x^{2}}+\phi(1-\phi)[a(x, t)+\phi]$ and in the deterministic differential equation $\frac{\partial \phi}{\partial t}=\frac{\partial^{2} \phi}{\partial x^{2}}+\phi(1-\phi)\left[a^{(0)}+\phi\right]$ at two different times [4]. The (dotted) shape of a step at $x=10$ denotes initial condition for both the deterministic and fluctuating fronts. The dashed lines correspond to the front shapes for the deterministic differential equation with $a^{(0)}=0.3$, and the solid lines correspond to the fronts in the stochastic differential equation, where $a(x, t)$ fluctuates around $a^{(0)}$ with an amplitude $\tilde{\varepsilon}=0.3\left(\Delta x=0.1, \Delta t=10^{-3}\right)$. The increased gap between the deterministic and the fluctuating front profiles at the later time is due to the increased front speed in the presence of the noise, as described in Eq. (76). Symbols have been changed from the original to keep consistency with the ones used here.

The method of separating these two different time scales goes back to the early 1980s [79,107], although it was carried out for weak noise and without the aid of Novikov's theorem. For weak noise one carries out a perturbative expansion in the noise strength $\tilde{\varepsilon}$, but the method to extract the diffusion coefficient of 
the so-called Goldstone mode arising out of the random displacement of the front from its uniformly translating position at long time scales, nevertheless, remains equally applicable. We will not derive the expressions for the diffusion coefficient of the so-called Goldstone mode for weak noise [79,94,107,108] here; instead we will summarize the key ideas of Refs. [79,94,107,108] in points (i)(iii) at the end of Sec. 3.1.2. Enthusiastic readers are encouraged to consult these references on their own to further satisfy their curiosity.

To analyze different phenomena taking place at different time scales, we first express $\phi$ in Eq. (72) as a combination of a fixed shape $\phi^{(0)}$ displaced by the amount $X(t)$ from its uniformly translating mean position $\xi=x-v_{\varepsilon} t$ and (time-dependent) fluctuations in the front shape $\delta \phi$ around the instantaneous position of the front as

$$
\phi(x, t)=\phi^{(0)}[\xi-X(t)]+\delta \phi[\xi-X(t), t] .
$$

It is precisely at this point where one first separates out the systematic and the fluctuating part of the front. As for the fluctuating part, a further separation of the effect of fluctuating term $\propto R(\phi, x, t)$ in Eq. (72) at two different time scales also takes place in Eq. (75). The fluctuations at long time scale is coded in the (random) wandering $X(t)$ of the Goldstone mode around $\xi=x-v_{\varepsilon} t$, whereas the fluctuations at short time scales in the front shape is coded in the fluctuations in the front shape, expressed by $\delta \phi[\xi-X(t), t]$. In this form, the shape $\phi^{(0)}$ as a function of its argument $\xi$ is obtained from the equation

$$
D \frac{\partial^{2} \phi^{(0)}}{\partial \xi^{2}}+v_{\varepsilon} \frac{\partial \phi^{(0)}}{\partial \xi}+f_{g}\left[\phi^{(0)}\right]=0 .
$$

As one can now see, the effective deterministic part now has a front solution that propagates with a speed $v_{\varepsilon}$ - the important thing to notice is that $v_{\varepsilon}$ is different from the speed of the deterministic front obtained by dropping the $\propto \eta(x, t)$ term of Eq. (61) altogether.

Clearly, in this process, the displacement of the front $X(t)$ from its uniformly translating mean position $\xi$ can be chosen in many different ways. One of the convenient ways is to obtain it through the so-called Goldstone mode. The idea is that just like in Eq. (15), if $\phi^{(0)}(\xi)$ is a solution of Eq. (76), then so it $\phi^{(0)}(\xi+a)$ for any $a$. This translational invariance of Eq. (76) indicates that $\Phi_{G, R}=\frac{\partial \phi^{(0)}}{\partial \xi}$ is the right eigenvector of the linear stability operator $\mathcal{L}_{v_{\varepsilon}}$ with eigenvalue zero, where

$$
\mathcal{L}_{v_{\varepsilon}}=\frac{\partial^{2}}{\partial \xi^{2}}+v_{\varepsilon} \frac{\partial}{\partial \xi}++\left.\frac{\delta f_{g}(\phi)}{\delta \phi}\right|_{\phi=\phi^{(0)}}
$$


The so-called Goldstone mode is simply the quantity $\Phi_{G, R}(\xi)[94,109,110]$, which also propagates with the speed $v_{\varepsilon}$ of the front solution $\phi^{(0)}(\xi)$.

As we already saw in Sec. 2.4.2, $\Phi_{G, L}(\xi)=e^{v_{\varepsilon} \xi / D} \frac{\partial \phi^{(0)}}{\partial \xi}$ is the left eigenvector of $\mathcal{L}_{v_{\varepsilon}}$ with eigenvalue zero, and then based on the idea of the Goldstone mode, we now uniquely define $X(t)$ from the relation $[79,94,97,107,108]^{65}$

$$
\int_{-\infty}^{\infty} d \xi e^{v_{\varepsilon} \xi / D} \frac{d \phi^{(0)}}{d \xi} \delta \phi[\xi-X(t), t]=0
$$

Thereafter, having used Eq. (75) in Eq. (72), at the lowest order of the magnitude of the front shape fluctuation, we have, on the instantaneous comoving frame of the Goldstone mode

$$
\frac{\partial(\delta \phi)}{\partial t}=\mathcal{L}_{v_{\varepsilon}} \delta \phi+\dot{X}(t) \frac{\partial \phi^{(0)}}{\partial \xi}+R(\phi, \xi, t),
$$

and then having left multiplied Eq. (79) with $\Phi_{G, L}(\xi)$ and integrating both sides w.r.t. $\xi$, we get

$$
\dot{X}(t)=-\tilde{\varepsilon}^{1 / 2}\left[\int_{-\infty}^{\infty} d \xi \Phi_{G, L}(\xi) R(\phi, \xi, t)\right] /\left[\int_{-\infty}^{\infty} d \xi \Phi_{G, L}(\xi) \Phi_{G, R}(\xi)\right] .
$$

The upshot of the entire exercise in terms of the usage of Novikov's theorem for propagating fronts is now clear - the stochastic differential equation (61) has a front speed $v_{\varepsilon}$, which has to be calculated from Eq. (76). On the other hand, the front speed also has (time-dependent) random fluctuations around $v_{\varepsilon}\left[\right.$ notice that $\left.\langle\dot{X}(t)\rangle_{\eta}=0\right]$.

One measure of the wandering of the entire front that is ubiquitous in literature on the external fluctuation effects on propagating fronts is the diffusion coefficient $D_{G}$ of the Goldstone mode. The quantity $D_{G}$, as can be easily guessed, is borne out of the random displacement $\dot{X}(t)$ at long time scales; but in order to obtain an expression for $D_{G}$ from Eq. (80), it is necessary that one replaces $R(\phi, \xi, t)$ by $R\left(\phi^{(0)}, \xi, t\right)$. This replacement makes $R \delta$-correlated both in space and time in Eq. (74), and then the front speed fluctuation becomes a Markov process originating from the fluctuating $R$ term in Eq. (72).

\footnotetext{
$\overline{65}$ For fluctuating fronts in reaction-diffusion systems, Eq. (78) is the operational definition of how to extract the conditionally averaged front profile from an ensemble of front realizations.
} 
Moreover, it also reduces the whole formalism of Langevin-type front evolution equation (61) with multiplicative noise in the Stratonovich interpretation to Eq. (72) with additive and white noise, resulting in the further reduction of the fluctuating "Langevin force" $R$ of variable magnitude in time to a force with a fixed magnitude. This reduction is absolutely necessary in order to even define $D_{G}$ [and also Eq. (84) in this form] for Stratonovich noise. ${ }^{66}$

By using the Kubo formula for the diffusion coefficient as the time integral of the autocorrelation function of the fluctuation in the front speed, and with the aid of Eq. (70) and (74) [the $O\left(\tilde{\varepsilon}^{1 / 2}\right)$ term in Eq. (74) is dropped], the diffusion coefficient $D_{G}$ of the Goldstone mode is obtained as

$$
D_{G}=\tilde{\varepsilon}\left[\int_{-\infty}^{\infty} d \xi \Phi_{G, L}^{2}(\xi) g^{2}\left[\phi^{(0)}\right]\right] /\left[\int_{-\infty}^{\infty} d \xi e^{v_{\varepsilon} \xi / D} \Phi_{G, R}^{2}(\xi)\right]^{2}
$$

Before we end Sec. 3.1.2, it is worth spending some time on three important issues. First, only after having derived Eq. (81), we can appreciate footnotes 64. The front speed $v_{\varepsilon}$ is obtained from Eq. (76), and to obtain $v_{\varepsilon}$, we have not carried out any expansion in powers of $\tilde{\varepsilon}$. On the other hand, for the diffusion coefficient $D_{G}$ of the Goldstone mode, we have dropped the $O\left(\tilde{\varepsilon}^{1 / 2}\right)$ term in Eq. (74). Despite that, the actual $\tilde{\varepsilon}$-dependence of $D_{G}$ is not simply the prefactor $\tilde{\varepsilon}$ on the r.h.s. of Eq. (81); in fact, there are other $\tilde{\varepsilon}$-dependent terms already occurring in the expression of $v_{\varepsilon}$ that enter both the numerator and as well as the denominator of Eq. (81) [3].

Secondly, the nomenclature "diffusion coefficient of the Goldstone mode" may a priori appear to be a misnomer - after all, since $D_{G}$ characterizes the wandering properties of the Goldstone mode, one would, quite rightfully, tend to think that $D_{G}$ should really be the front diffusion coefficient. Why the name "diffusion coefficient of the Goldstone mode" has been chosen in this review article is in a way related to my own personal choice of words. It turns out that $D_{f}$ for the front defined earlier is in fact conceptually totally different from the field-theory expression (81). While we leave further details on this point till Sec. 4.2, to differentiate these two quantities, we will continue to use the terminology "diffusion coefficient of the Goldstone mode" for Eq. (81).

Thirdly, the front shape fluctuations around the instantaneous position of the front at fast time scales can also be analyzed by defining the (mutually orthonormal) shape fluctuation modes $\left\{\Psi_{m}(\xi)\right\}$ in the eigenspace of non-zero eigenvalues of the linearized operator $\mathcal{L}_{v_{\varepsilon}}$ as

\footnotetext{
${ }^{66}$ We will later see in Sec. 4 that when $R(\phi, \xi, t)$ is interpreted in the Itô sense, $D_{G}$ can be well-defined without replacing $R(\phi, \xi, t)$ by $R\left(\phi^{(0)}, \xi, t\right)$. See also Sec. 3.5.
} 


$$
\delta \phi(\xi, t)=\sum_{m \neq 0} c_{m}(t) \Psi_{m, R}(\xi)
$$

Here $\Psi_{m, R}(\xi)$ is the right eigenvector of $\mathcal{L}_{v_{\varepsilon}}$ with eigenvalue $\tau_{m}^{-1}$, and $\Psi_{m, L}(\xi)=$ $e^{v_{\varepsilon} \xi / D} \Psi_{m, R}$ is the corresponding left eigenvector. In the appropriate context, $e^{v_{\varepsilon} \xi /(2 D)} \Psi_{m, R}$ is basically the same as $\psi_{m}(\xi)$ encountered in Eq. (21). Needless to say,

$$
\int_{-\infty}^{\infty} d \xi \Psi_{m, L}(\xi) \Phi_{G, R}(\xi)=0, \quad \forall m
$$

i.e., each of these modes is orthogonal to the Goldstone mode. The mode expansion (82) then easily leads one to

$$
\dot{c}_{m}(t)=-\tau_{m}^{-1} c_{m}+\tilde{\varepsilon}^{1 / 2} \int_{-\infty}^{\infty} d \xi \Psi_{m, L}(\xi) R\left(\phi^{(0)}, \xi, t\right)
$$

and its corresponding solution

$$
c_{m}\left(t+t^{\prime}\right)=e^{-\frac{t^{\prime}}{\tau_{m}}} c_{m}(t)+\tilde{\varepsilon}^{1 / 2} \int_{0}^{t^{\prime}} d t^{\prime \prime} \int_{-\infty}^{\infty} d \xi e^{-\frac{t^{\prime}-t^{\prime \prime}}{\tau_{m}}} \Psi_{m, L}\left(\xi^{\prime}\right) R\left(\phi^{(0)}, \xi, t^{\prime \prime}\right) .
$$

Notice that in Eqs. (82-85), we have first used $\psi_{m}(\xi)=e^{v_{\varepsilon} \xi /(2 D)} \Psi_{m, R}(\xi)$ to first convert $\mathcal{L}_{v_{\varepsilon}}$ to a Hermitian operator [see Eqs. (16) and (17)], of which $\psi_{m}(\xi)$ is the eigenvector with eigenvalue $\tau_{m}^{-1}$. We then implicitly made use of the completeness condition for $\left\{\psi_{m}(\xi)\right\}$ :

$$
\sum_{m \neq 0} \psi_{m}(\xi) \psi_{m}\left(\xi^{\prime}\right)=\delta\left(\xi-\xi^{\prime}\right)
$$

This completeness condition also means that $c_{m}(t)$ at any time $t$ is automatically determined from the following equation:

$$
c_{m}(t)=\int_{-\infty}^{\infty} d \xi \Psi_{m, L}(\xi) \delta \phi(\xi, t)
$$

We will not need Eqs. (82-87) in Sec. 3; however, they will come handy for Sec. 4.2 . 
We finally end Sec. 3.1 with the remark that to derive Eqs. (61-81) for weak noise $(\tilde{\varepsilon} \ll 1)$ and for the cases when $\eta(x, t)$ does not necessarily satisfy Eq. (65) $[79,94,107,108]$, using small noise expansion formulation [45], $\phi^{(0)}$ comes out to be the same as $\phi^{*}$ or $\phi^{\dagger}$ (correspondingly with $v_{\varepsilon}=v^{*}$ or $v^{\dagger}$ ), depending on whether the deterministic part of Eq. (61) gives rise to a pulled or a pushed front. This implies that in these approaches, while there is no steady modification in the front speed due to the presence of the noise, the only new phenomenon that the noise gives rise to is the diffusive wandering of the Goldstone mode. Nevertheless, there are a few interesting results derived in Refs. [79, 94, 107, 108] for weak noise, and we summarize them in points (i) through (iii) below.

(i) A derivation of the diffusion coefficient of the Goldstone mode for general noise correlations can be found in Refs. [79, 107]. An application to derive an exact result for $D_{G}$ for front propagation in bistable systems can be found in Ref. [79] when $\left\langle\eta(x, t) \eta\left(x^{\prime}, t^{\prime}\right)\right\rangle_{\eta} \propto \exp \left[\frac{-\left(t-t^{\prime}\right)^{2}}{\tau_{c}^{2}}\right] \exp \left[\frac{-\left(r-r^{\prime}\right)^{2}}{\lambda_{c}^{2}}\right]$ with characteristic time and length scale $\tau_{c}$ and $\lambda_{c}$ respectively.

(ii) The probability density of $X(t)$ based on the dynamics similar to Eq. (80) has been analyzed in Ref. [108]. An application to derive an exact result for $D_{G}$ for front propagation in a bistable system can be found therein when $\eta(x, t)$ satisfies Eq. (65).

(iii) A treatment of how to calculate the correlation function $\left\langle\phi(x, t) \phi\left(x^{\prime}, t\right)\right\rangle_{\eta}$ exists in Ref. [94]. The theory was then applied to front propagation in a bistable system and to front propagation into an unstable state (of a Fisher equation type model).

\subsection{Application of Novikov's Theorem to Reaction-Diffusion Systems: Effects of Multiplicative Noise on Specific Models}

Equipped with the necessary groundwork in Sec. 3.1.1, we now analyze the effect of multiplicative noise on specific models of reaction-diffusion systems.

\subsubsection{An Example of (Multiplicative) Noise-Induced Front Transitions}

Consider the reaction-diffusion equation

$$
\frac{\partial \phi}{\partial t}=D \frac{\partial^{2} \phi}{\partial x^{2}}+\phi(1-\phi)\left[a^{(0)}+\phi\right]
$$


with three homogeneous stationary states, $\phi=0,-a^{(0)}$ and 1 . To provide the state $\phi=1$ with global stability, we confine $a^{(0)}$ within the range $[-1 / 2,1]$ with this restriction on the value of $a^{(0)}$, one arrives back at Eq. (54).

The interesting point to note in this reaction-diffusion equation is that it admits front solutions whose characteristics are different for different values of $a^{(0)}$, as we illustrate below in (a)-(c).

(a) $-1 / 2 \leq a^{(0)}<0$ : In this range, $\phi=0$ state is metastable. The linear marginal stability criterion does not hold in this range, and a front solution propagating with speed $v^{\dagger}=\sqrt{D}\left[2 a^{(0)}+1\right] / \sqrt{2}$ emerges uniquely. Let us denote this phase of the front by $\mathrm{M}$.

(b) $0 \leq a^{(0)}<1 / 2$ : In this range, $\phi=0$ is linearly unstable, but for sufficiently localized initial conditions, the front speed is still given by $v^{\dagger}$. For less localized initial conditions, front speeds higher than $v^{\dagger}$ are also accessible. We denote this phase of the front by NL.

(c) $1 / 2 \leq a^{(0)} \leq 1$ : Finally, linear marginal stability holds in this range. For sufficiently localized initial conditions, a pulled front solution propagating with speed $v^{*}=2 \sqrt{D a^{(0)}}$ emerges. For less localized initial conditions, front speeds higher than $v^{*}$ is also accessible. This phase of the front is denoted by L.

How multiplicative noise can affect the characteristics of a front in a reactiondiffusion system is beautifully illustrated when $a^{(0)}$ in Eq. (88) is replaced by a fluctuating quantity $a(x, t)=a^{(0)}+\tilde{\varepsilon}^{1 / 2} \eta(x, t)[3,4]$, yielding

$$
\frac{\partial \phi}{\partial t}=D \frac{\partial^{2} \phi}{\partial x^{2}}+\phi(1-\phi)[a(x, t)+\phi]
$$

Here, $\eta(x, t)$ is a Gaussian random function - in the limit of correlation length and time of the noise going to zero, $\eta(x, t)$ satisfies Eq. (65).

The application of Novikov's theorem to this model yields the equivalent of Eq. (76), expressed as [3,4]

$$
D \frac{\partial^{2} \phi}{\partial x^{2}}+v_{\varepsilon} \frac{\partial \phi}{\partial x}+\phi(1-\phi)\left[a^{(0)}+\varepsilon+(1-2 \varepsilon) \phi\right]=0
$$

In the presence of noise, this modification of the front equation from Eq. (88) reorganizes the characteristics of the front - effectively, it is this reorganization that ends up being termed as the noise-induced front transition. The idea behind this transition is simple: just like the characteristics of the uniformly translating front solution of Eq. (88) depend on the value of $a^{(0)}$, the characteristics of the front solution (90) for a given value of $a^{(0)}$ depends on $\varepsilon$. 


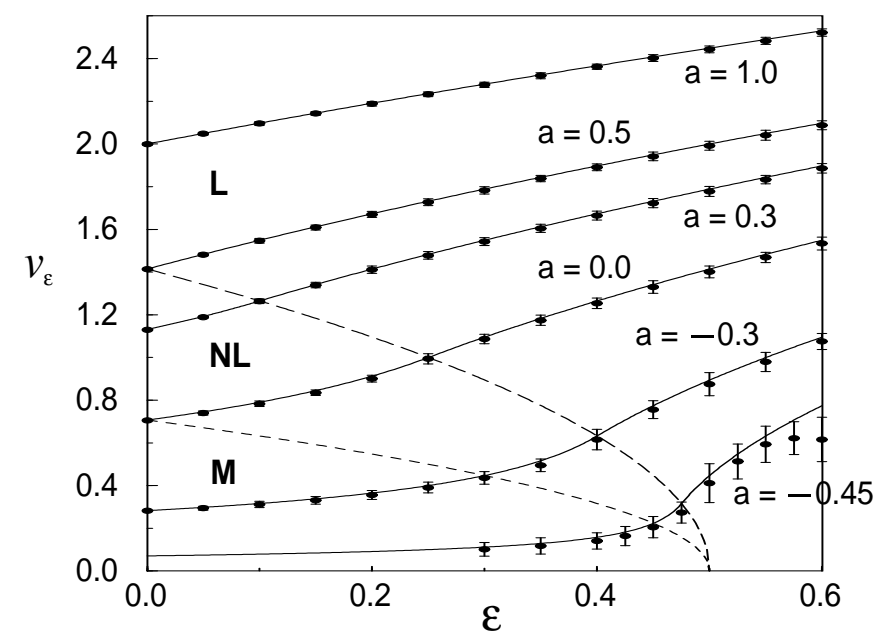

Fig. 28. Noise-induced front transition in Eq. (90) for $D=1[3,4]$. Solid lines: theoretical values for the front speed $v_{\varepsilon}=2 \sqrt{D\left(a^{(0)}+\varepsilon\right)}$ (L phase) and $v_{\varepsilon}=\left(2 a^{(0)}+1\right) \sqrt{D /[2(1-\varepsilon)]}$ (NL and M phase). Filled circles with error bars: simulation data. Symbols have been changed from the original to keep consistency (also the figure has been modified for greater clarity), and $a$ should be read as $a^{(0)}$.

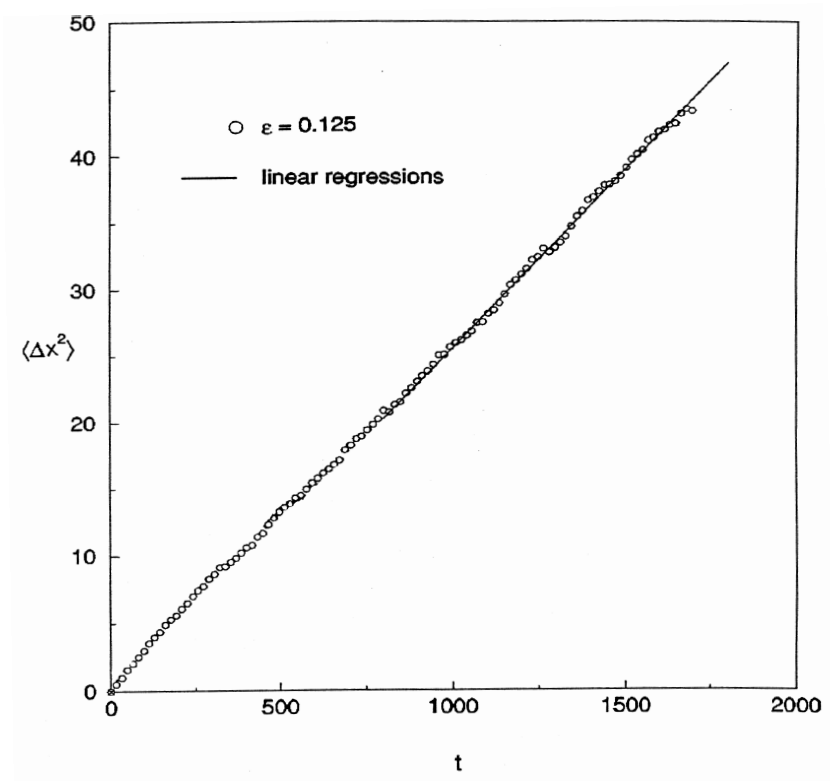

Fig. 29. Wandering behaviour of the Goldstone mode for $D=1, a^{(0)}=0.1$, $\Delta x=0.5, \Delta t=0.1$, and $\varepsilon=0.125$ [3]. The graph has been modified from its original for clarity. The notation $\Delta x$ appearing in the $y$-axis should not be confused with the discretization unit $\Delta x$.

This implies that by adjusting the value of $\varepsilon$, it is possible to convert a front solution of Eq. (88) that belongs to one of the M, NL or L phases to a front solution (90) belonging to another one of these phases. For sufficiently steep initial conditions, in the $\mathrm{L}$ phase, the front (90) propagates with the speed $2 \sqrt{D\left(a^{(0)}+\varepsilon\right)}$, while both in the NL and in the M phase, the front propagates 
with the speed $\left(2 a^{(0)}+1\right) \sqrt{D /[2(1-\varepsilon)]}$. In fact, the boundary between the $\mathrm{NL}$ and the $\mathrm{L}$ phase for the front solution (90) lies at $\varepsilon=1 / 4-a^{(0)} / 2 .{ }^{67}$

The $v_{\varepsilon}$ vs. $\varepsilon$ plots for Eq. (90) [3,4] are shown in Fig. 28. Simulation results for the wandering behaviour of the Goldstone mode for $D=1, a^{(0)}=0.1$, $\Delta x=0.5, \Delta t=0.1$, and $\varepsilon=0.125[3]$ appear in Fig. 29 .

\subsubsection{An Example of (Multiplicative) Noise-Induced Fronts}

In Sec. 3.2.1, we considered a system that already admits a front solution in the absence of noise, and witnessed how the characteristics of the front properties can change when multiplicative noise is introduced in it. We now consider a system that does not admit a propagating front solution in the absence of noise, but when the amplitude of the external noise exceeds a threshold value, fronts start to propagate (see Fig. 30). In the front propagation literature, such a front is known as a noise-induced front. An example system that exhibits such noise-induced fronts is [105]

$$
\frac{\partial \phi}{\partial t}=D \frac{\partial^{2} \phi}{\partial x^{2}}-\phi\left[a(x, t)+\phi^{2}\right]
$$

Clearly, when $a(x, t)$ is replaced by $a^{(0)}>0$ in Eq. (91), the equation does not admit a propagating front solution - any perturbation around the state $\phi=0$ simply decays in time. Elsewhere in the literature, this phenomenon is known as propagation failure. Similarly, the emergence of propagating front solutions in Eq. (91) when $a(x, t)$ becomes a sufficiently strongly fluctuating quantity is known as the breakdown of propagation failure (see e.g. Ref. [57]). In fact, the situation is comparable to the so-called stochastic resonance paradigm [2].

With $a(x, t)=a^{(0)}+\tilde{\varepsilon}^{1 / 2} \eta(x, t)$, where $\eta(x, t)$ is a Gaussian random function whose correlation properties reduce to Eq. (65) in the limit of infinitesimal correlation time and length, the equivalent of Eq. (76) obtained with the application of Novikov's theorem is expressed as [105]

$$
D \frac{\partial^{2} \phi}{\partial x^{2}}+v_{\varepsilon} \frac{\partial \phi}{\partial t}-\phi\left[a^{(0)}-\varepsilon+\phi^{2}\right]
$$

It is clear from Eq. (92) that when $\varepsilon>a^{(0)}$, fronts propagate from the stable

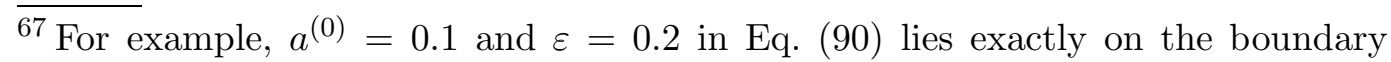
between the NL and the L phase. Later in Sec. 3.3, we will see that this boundary between the NL and the L phase marks a change in the wandering properties of the Goldstone mode as well. 
state $\phi_{\text {st }}=\sqrt{D\left[\varepsilon-a^{(0)}\right]}$ to the linearly unstable state $\phi=0$ with the (pulled) speed $v_{\varepsilon}=2 \sqrt{D\left[\varepsilon-a^{(0)}\right]}$ (see Fig. 31).

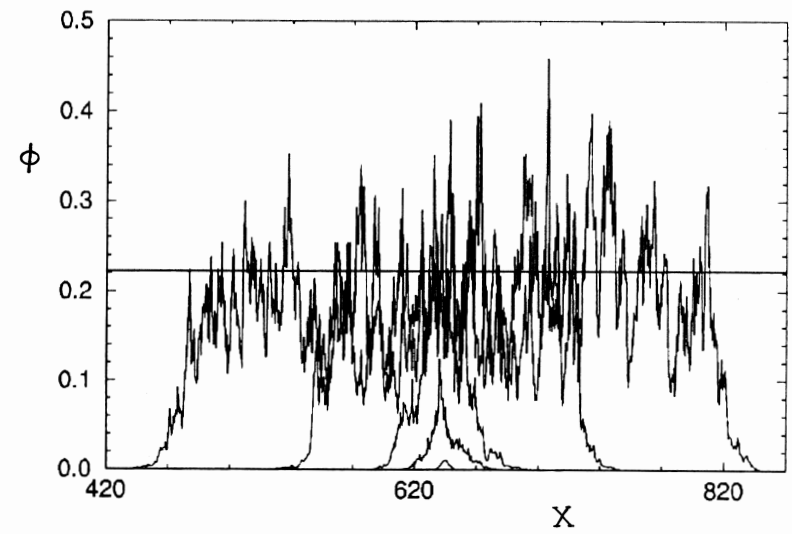

Fig. 30. Snapshots of front configurations for noise-induced front propagation in Eq. (91) [with $D=1$ ] when $a(x, t)$ fluctuates around $a^{(0)}=0.1$ with an amplitude $\tilde{\varepsilon}=0.15[105]$. The snapshots have been taken at $t=50,100,240$ and 450 .
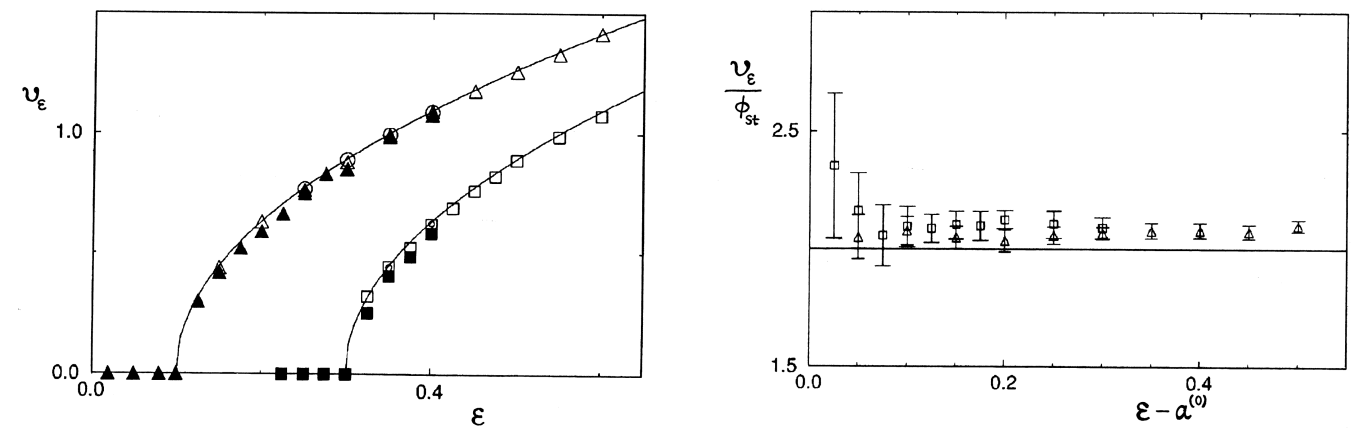

Fig. 31. Noise-induced front propagation in the reaction-diffusion system (91) [105]. Left figure: $v_{\varepsilon}$ vs. $\varepsilon$ for $D=1$ and two different values of $a^{(0)}$ : namely $a^{(0)}=0.1$ (triangles), and $a^{(0)}=0.3$ (squares). Right figure: theoretically predicted value of $v_{\varepsilon} / \phi_{\text {st }}$ vs. simulation for $D=1$. Filled symbols: simulations of the stochastic differential equation (91) for space and time discretization units $\Delta x=0.5$ and $\Delta t=0.01$ respectively; open symbols: $\Delta x=0.1$ and $\Delta t=0.001$. Circles represent front solution (92), and lines denote the theoretical prediction $v_{\varepsilon}=2 \sqrt{D\left[\varepsilon-a^{(0)}\right]}$.

\subsection{Fluctuating Pulled Fronts with Multiplicative Noise in Reaction-Diffusion Systems: Diffusive vs. Subdiffusive Wandering of the Goldstone Mode}

In footnote 67 , we had commented that for the reaction-diffusion model (88) at $a^{(0)}=0.1$ and $\varepsilon=0.2$, the wandering of the Goldstone mode is subdiffusive, and also that this phenomenon is caused by the fact that the $a^{(0)}=0.1, \varepsilon=$ 0.2 sits precisely on the boundary between the L and the NL phase. Truly 
speaking, the above argument is misleading - contrary to what may appear from footnote 67 , this subdiffusive behaviour has nothing to do with noiseinduced front transitions. Instead, the root of this subdiffusive behaviour turns out to go deeper - as soon as a fluctuating pulled front ${ }^{68}$ with multiplicative noise belongs to the $\mathrm{L}$ phase, the understanding of the wandering properties of the corresponding Goldstone mode calls for an entirely different approach [98].

The reason for the requirement that a different approach is needed to study the wandering properties of the fluctuating pulled fronts is not difficult to trace; after all, for these fronts it is pretty clear that one runs into trouble with Eq. (81). The point is that for them the (linearized) semi-infinite leading edge of $\phi^{(0)}(\xi)$ in Eq. (76) behaves as $\xi \exp \left[-v_{\varepsilon} \xi /(2 D)\right]$. This implies that for $\xi \rightarrow \infty$, at the leading order, the corresponding Goldstone modes $\Phi_{G, R} \equiv \frac{d \phi^{(0)}(\xi)}{d \xi}$ and $\Phi_{G, L} \equiv e^{v_{\varepsilon} \xi / D} \frac{d \phi^{(0)}(\xi)}{d \xi}$ behave as $\sim \xi \exp \left[-v_{\varepsilon} \xi /(2 D)\right]$ and $\sim \xi \exp \left[v_{\varepsilon} \xi /(2 D)\right]$ respectively, which then send the magnitude of both the numerator and the denominator in Eq. (81) to $\infty$.

In the next paragraph, following Ref. [98], we will argue that the wandering width $\mathcal{W}(t)=\sqrt{\left\langle X^{2}(t)\right\rangle}$ of the Goldstone mode for fluctuating pulled fronts with multiplicative noise scales as $t^{1 / 4}$ at long times. A more rigorous derivation of this result by means of converting Eq. (88) for $a^{(0)}=1$ to a KPZ equation for a one-dimensional interface in terms of the variable $h(\xi, t)$, defined by the Cole-Hopf transformation $\phi(x, t)=\exp \left[h(\xi, t)-v_{\varepsilon} \xi /(2 D)\right]$, can also be found in Ref. [98]. We will summarize this rigorous method later, but will not elaborate on it.

The argument for the $t^{1 / 4}$ scaling behaviour of $\mathcal{W}(t)$ at long times [98] is the following: notice that before even one arrives at Eq. (81), a more severe problem already occurs at the level of Eq. (75) for fluctuating pulled fronts - namely that when $\phi^{(0)}$ is pulled, then there is no inherent time scale for the convergence of the front speed to its asymptotic value [c.f. Eq. (4)]. We had earlier seen that this behaviour originate from the gapless spectrum of the stability operator (77) for pulled fronts. It implies that separating the effect of the fluctuations into a "slow" wandering of the Goldstone mode from the "fast" modes of fluctuations in the front shape, upon which the whole underlying philosophy of Eqs. (75) through (81) is based, does not make sense any longer. Nevertheless, the $t^{1 / 4}$ scaling behaviour of $\mathcal{W}(t)$ emerges beautifully when the long-time behaviour of $\phi^{(0)}(\xi, t)$ is successfully married to Eq. (81), and that

\footnotetext{
$\overline{{ }^{68} \text { What }}$ we mean by fluctuating pulled front here is a fluctuating front with multiplicative noise belonging to the L phase. Here our usage of the phrase fluctuating pulled front is chosen to distinguish these fronts from the fluctuating "pulled" fronts that we encountered in Sec. 2.
} 
is carried out in the following manner.

With the result that the leading edge of the pulled front $\phi^{(0)}(\xi)$ relaxes asymptotically as [38]

$$
\phi^{(0)}(\xi, t) \sim \xi e^{-v_{\varepsilon} \xi /(2 D)-\xi^{2} /(4 D t)} / t^{3 / 2} \text { for } \xi, t \gg 1
$$

one defines a time-dependent upper cutoff $\xi_{c} \sim \sqrt{4 D t}$ for the $\xi$ integral in the denominator of Eq. (81). When this upper cutoff is inserted in Eq. (81), one obtains a time-dependent diffusion coefficient for the Goldstone mode [98]

$$
D_{G} \sim \frac{3 \tilde{\varepsilon}}{v_{\varepsilon}^{2} \sqrt{\pi D t}}
$$

for $t \gg 1$. The implication of Eq. (94) then is that for $t \gg 1, \mathcal{W}(t)$ could possibly scale as $t^{1 / 4}$.

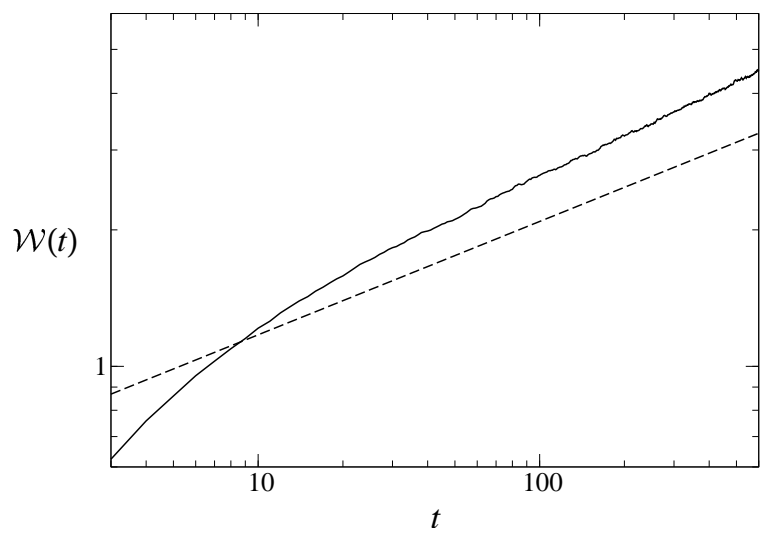

Fig. 32. Log-log plot of $\mathcal{W}(t)$ vs. $t$ showing subdiffusive wandering of the Goldstone mode for fluctuating pulled fronts with multiplicative noise (89) [98]. The data correspond to $a^{(0)}=1$. Solid line: simulation data, dashed line: a line with slope $1 / 4$.

To obtain the $t^{1 / 4}$ scaling of $\mathcal{W}(t)$ in terms of the rigorous Cole-Hopf transformation of Eq. (89) to a one-dimensional KPZ equation [98], one invokes the scaling relation $\mathcal{W}(t)=t^{\alpha} F\left(t / L^{\beta}\right)$. When the argument $\mu$ of $F(\mu)$ is $\ll 1$, then $F(\mu)$ behaves like a constant, and when $\mu \gg 1$, then $F(\mu) \sim \mu^{-\alpha}$. The proper way to implement the above scaling relation is to use a time-dependent length scale $L(t) \sim \xi_{c} \sim \sqrt{4 D t}$. For a one-dimensional interface, the KPZ exponents are $\alpha=1 / 3$ and $\beta=3 / 2$, which indicates that for $t \rightarrow \infty$, the argument of $F$ also approaches $\infty$. The long time behaviour of $\mathcal{W}(t)$ is then clearly given by $t^{1 / 3}\left[t /(\sqrt{t})^{3 / 2}\right]^{-1 / 3}=t^{1 / 4}$.

The long time $t^{1 / 4}$ scaling behaviour of $\mathcal{W}(t)$ for the model (88) with $a^{(0)}=1$ has been confirmed by numerical simulations [98] (see Fig. 32). We note here 
that although the wandering properties of the Goldstone mode for the model (91) was not studied in Ref. [105], from the argument based on the upper cutoff $\xi_{c}$ for the integrations in Eq. (81), in this model too we expect the same $t^{1 / 4}$ scaling behaviour for $\mathcal{W}(t)$ at long times. A rigorous derivation of it based on the Cole-Hopf transformation in the spirit of Ref. [98] is left for the enthusiastic readers.

\subsection{Fluctuating Fronts with Multiplicative Noise in Reaction-Diffusion Sys- tems and Kinetic Roughening}

While the Cole-Hopf transformation allows one to rigorously derive the $t^{1 / 4}$ scaling of the wandering properties of the Goldstone modes of fluctuating pulled fronts with multiplicative noise in one spatial dimension, questions can certainly be raised regarding its applicability to higher dimensions. Surprisingly enough, it turns out that the Cole-Hopf transformation is equally successful for reaction-diffusion systems in two spatial dimensions, only if for $\xi \rightarrow \infty$, the multiplicative noise couples linearly to the front field. Such a reaction-diffusion equation

$$
\frac{\partial \phi}{\partial t}=D \nabla^{2} \phi+[1+\sqrt{\tilde{\varepsilon}} \eta(x, t)] \phi\left[1-\phi^{2}\right],
$$

in two spatial dimensions has been considered in Ref. [115]. The variable $h$ then corresponds to a two-dimensional interface (see Fig. 33). The front wandering properties in Eq. (95) has then been shown to obey the KPZ scaling for a two-dimensional interface ${ }^{69}$ - see Fig. $34 .{ }^{70}$

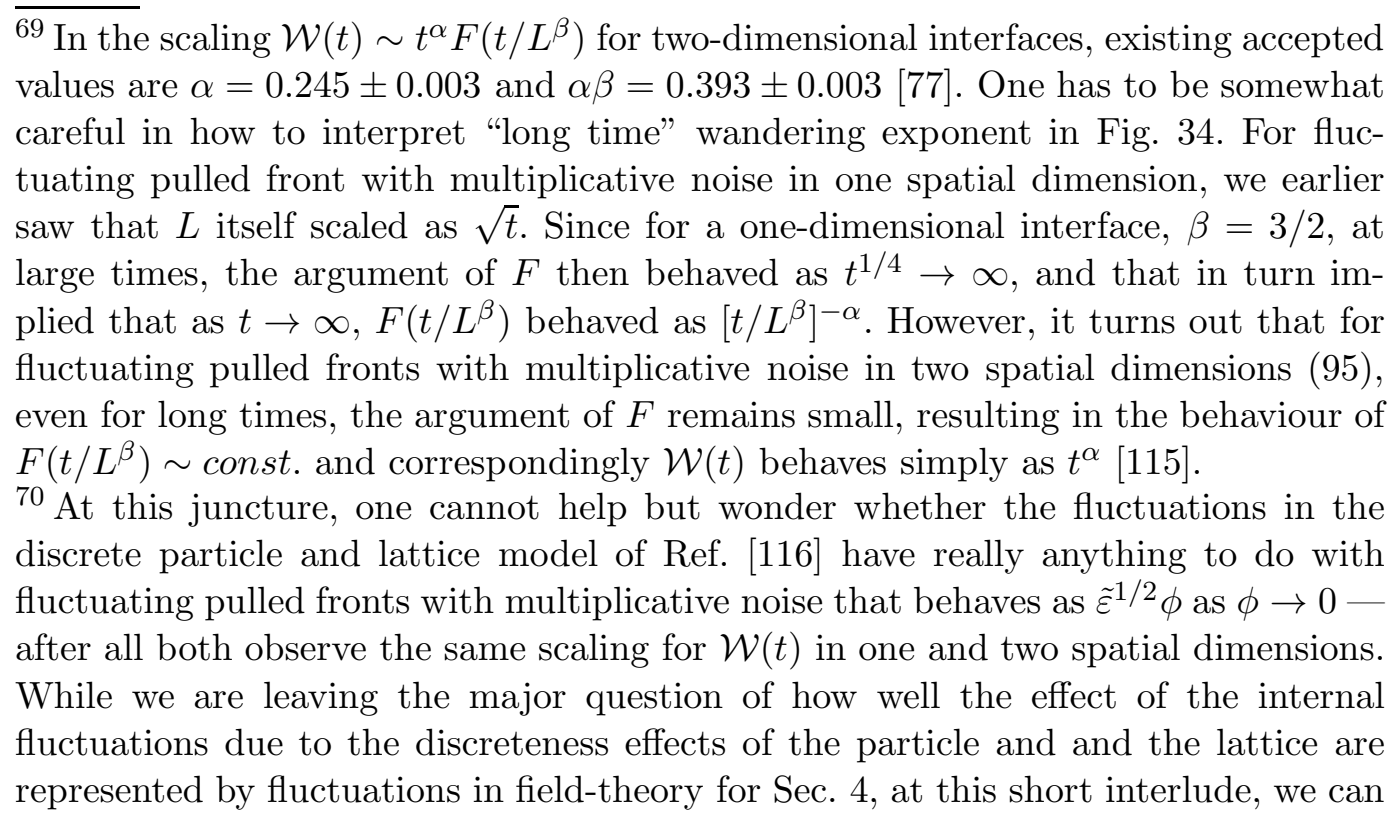



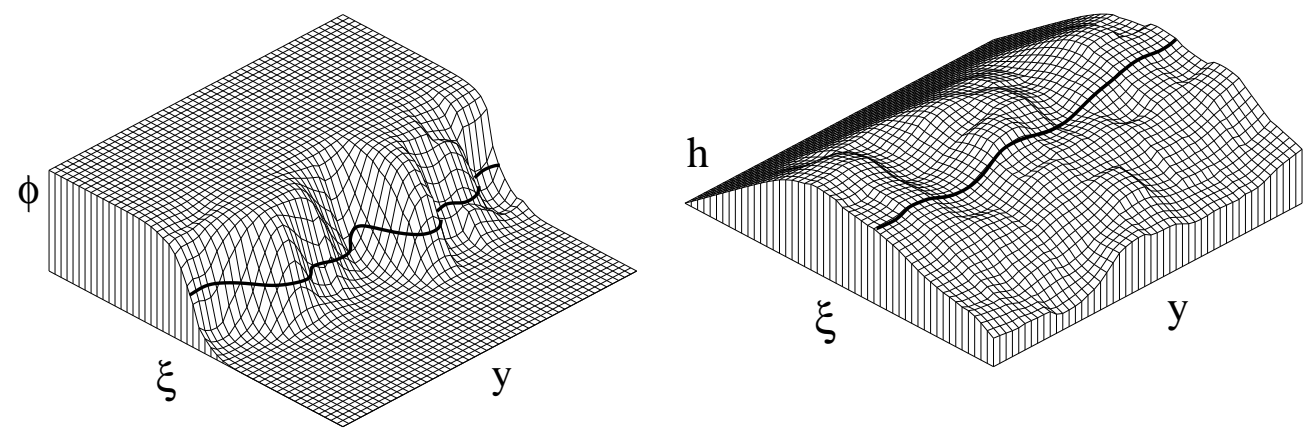

Fig. 33. Fluctuating pulled front propagation with multiplicative noise in two spatial dimensions for the model (95). The front propagates in the $x$ direction, and $\xi=x-v_{\varepsilon} t$. Left figure: an instantaneous snapshot of the front configuration. Right figure: the corresponding configuration in terms of the two-dimensional interface "height" $h(\xi, t)$ obtained by the Cole-Hopf transformation. The solid curve denotes, in both figures, the contour for position $(\xi, y)$ where the value of $\phi$ is $1 / 2$.

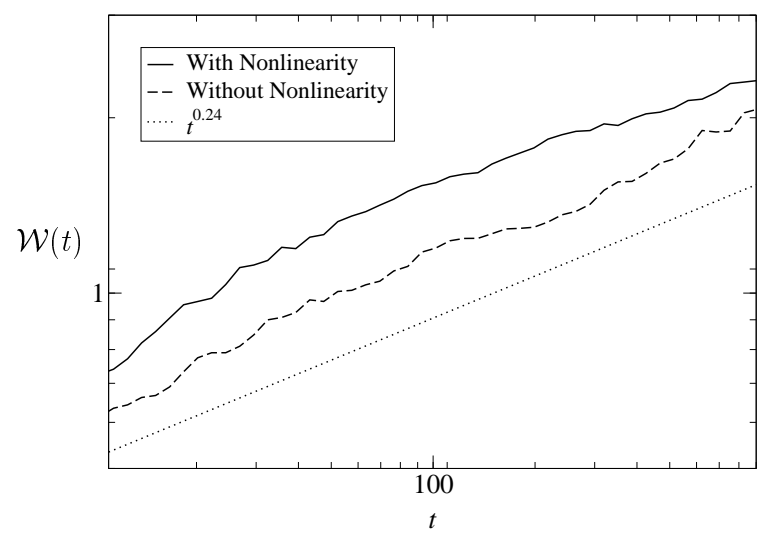

Fig. 34. Log-log plot of $\mathcal{W}(t)$ vs. $t$ showing the wandering of the Goldstone mode for the fluctuating pulled front with multiplicative noise in Eq. (95) [115]. The with and without nonlinearity respectively indicate whether nonlinear terms $\propto \exp [h(\xi, t)]$ generated through the Cole-Hopf transformation of Eq. (95) were retained in the simulation or not.

The success of the Cole-Hopf transformation to reduce reaction-diffusion equations with multiplicative noise in $d$ spatial dimensions to a KPZ equation for a $d$ dimensional interface is certainly extremely intriguing. Although in any spatial dimensions, this transformation works only if for $\xi \rightarrow \infty$, the fluctuations in the external parameter linearly couples to the front field, one wonders if there exist further general connections between the evolution equations of fronts and those describing interfacial growth phenomena. From this perspective, a beautiful analysis for fronts (even with vector front fields) in reactiondiffusion equations in arbitrary dimensions has been presented in Ref. [99].

say that the results of Ref. [80] points the finger to a negative answer. We will further see in Sec. 4.1 that the internal fluctuations are usually modeled by a fluctuating term in the stochastic differential equation that behaves as $\sqrt{\phi}$ for $\phi \rightarrow 0$, which too, rules out an affirmative possibility. 
The analysis is not rich in mathematical rigour, and we will not describe its mathematical procedure here either. The idea therein is the same as what has been already presented in Sec. 3.1.2; namely that one needs to perform a separation of length and time scales. As the short time and length scales are averaged out by means of a coarse graining method, leaving behind the long length and time scales in a "renormalized" evolution equation of the front, one does recover the dynamical equations for growing interfaces, standardly seen in interfacial growth literature.

Applied to specific systems of Sec. 3.2.1, this formalism yields the same results for the expression of the shift in front speed and the diffusion coefficient of the Goldstone mode in one dimension, as expected [99]. It also reproduces the front fluctuation characteristics in Fig. 34 for the reaction-diffusion system (95) in two dimensions.

\subsection{Epilogue II}

Generally speaking, (Langevin-type) field-theoretical methods have been used ubiquitously by chemists to describe the effect of external noise on chemical reaction-diffusion systems for decades. In many of these chemical systems, one does observe front propagation. From that point of view, it has been difficult for me to decide, for Sec. 3, what to significantly include, what to mention in appropriate context and what to leave out. In fact, in Sec. 3, I have adopted the philosophy of discussing only those papers in detail that have contributed significantly to the field-theoretical framework on the effect of external noise on propagating fronts. In this process, I have strived to provide a unified perspective of these field-theoretical methods.

While all the works that I am aware of on these field-theoretical methods have exclusively been on reaction-diffusion systems, quite expectedly, there exist a few scattered results that concerned weak noise, or simply additive fluctuations. These results, in addition to some of the real laboratory experiments that in the first place motivated the field-theoretical studies of multiplicative noise on propagating fronts can be traced by following the cited references in the works that we have discussed in Sec. 3.

There is one further point to that I would like to bring the reader's attention to. That is the fact that effectively majority of the works on field-theoretical treatments of propagating fronts with multiplicative noise considered only spatiotemporally white noise - after all, spatiotemporal white noise in the ensuing analytics is a lot easier to handle than coloured or structured noise. Although we started with a fancier form of the noise (62) in Sec. 3, we reduced it to spatiotemporal white noise (65) very soon in the limit of both $\lambda_{c}$ and 
$\tau_{c} \rightarrow 0$.

This however does not imply, in any way, that structured noise forms have not been considered in the literature to study the effect of multiplicative noise on propagating fronts. Few examples for weak coloured noise forms have been mentioned in points (i)-(iii) at the end of Sec. 3.1.2. A recent work has appeared with spatiotemporally structured (and not necessarily weak) noise [106] - wherein an approach along the lines of Novikov's theorem ${ }^{71}$ [106] has been adopted. The central theme of this line of thought is, once again, to rewrite Eq. (61) in the form of Eq. (72), such that the stochastic term does have a zero mean. In the absence of the applicability of Novikov's theorem, in this case, one carries out a cumulant expansion of the noise term to evaluate the l.h.s. of Eq. (70) [106] (the precise mathematical procedure in this cumulant expansion is non-trivial; but naturally, in the limit of both $\tau_{c}$ and $\lambda_{c} \rightarrow 0$ one recovers the white noise results). The limitation of this technique, however, is that it is a perturbative expansion in (small) correlation length and time, and therefore, one should not expect more than some limited success.

Before we end Sec. 3, let us return to the paragraph below Eq. (84). We had commented therein that $D_{G}$ is defined only when $R(\phi, \xi, t)$ is replaced by $R\left(\phi^{(0)}, \xi, t\right)$ in Eq. (79). While Eq. (80) is still well-defined without this replacement, Eq. (81) is not. First of all, note that without having $R(\phi, \xi, t)$ replaced by $R\left(\phi^{(0)}, \xi, t\right), R$ is no longer white both in space and time [c.f. Eq. (74)], and that certainly gets one into trouble. Secondly (and more importantly), taking the average over the noise alone to obtain an expression of $D_{G}$ is not enough.

To make the second point more articulate, let us take a note of the fact that the average over the noise, such as in Eq. (74), implies that for $t^{\prime}>t$, one averages over all possible noise realizations after $t$. With this average alone, the expression for $D_{G}$ [without having $R(\phi, \xi, t)$ replaced by $R\left(\phi^{(0)}, \xi, t\right)$ ] depends on the precise front configuration at time $t$. Thereafter, one still has to also average over the ensemble of realizations of the front configurations at time $t$ [just like in Eq. (28)] to properly define $D_{G}$. In that case, to obtain a corresponding theoretical expression of $D_{G}$ for general noise terms is not an easy task (we will see more of it in Sec. 4.2.1.

Nevertheless, whether one can replace $R[\phi(\xi, t), \xi, t]$ by $R\left[\phi^{(0)}(\xi, t), \xi, t\right]$ under general conditions is a natural question. A priori, it seems to be a trivial issue, because for weak noise, one is always accustomed to think that such a replacement is always possible. The point however is that since we are dealing with fluctuating fronts that propagate into unstable states, i.e., $f_{g}(\phi) \sim \phi$ for $\phi \rightarrow 0$, one must always bear in mind the following note of caution: in the limit

\footnotetext{
${ }^{71}$ Recall that Novikov's theorem is applicable only when the noise is Gaussian. This requirement renders it unusable for coloured noise with non-Gaussian statistics.
} 
of weak noise, so long as $R[\phi(\xi, t), \xi, t] / \phi \rightarrow 0$ for $\phi \rightarrow 0$ (all the cases that we discussed in Sec. 3 follow this property), $R[\phi(\xi, t), \xi, t]$ can be replaced by $R\left[\phi^{(0)}(\xi, t), \xi, t\right]$. Indeed, in Sec. 4 , we will see this condition violated, namely that for the sFKPP equation (96) $R[\phi(\xi, t), \xi, t] / \phi \not \rightarrow 0$ for $\phi \sim 1 / N$ and $N \rightarrow \infty$. In that case, if one does replace $R[\phi(\xi, t), \xi, t]$ can be replaced by $R\left[\phi^{(0)}(\xi, t), \xi, t\right]$, one gets a front diffusion coefficient scaling as $1 / N$, which is certainly wrong!

\section{Field-theory of Fluctuating Fronts: Internal Fluctuations}

In first three paragraphs of Sec. 3, we have already got a taste of the complications associated with internal fluctuations. It therefore should not come as a surprise that (unlike what we experienced in the field-theory of fluctuating fronts for external fluctuations) describing the effect of internal fluctuations on propagating fronts is a real challenge for the field-theorists. Before we plunge into the details in Sec. 4.1 onwards, let us demonstrate the major intricacies.

To start with, let us remind ourselves that all the works on the field-theoretical treatments of the effects of internal fluctuations on propagating fronts that I am aware of have been exclusively in the reaction-diffusion systems. However, as far as Sec. 4 is concerned, the readers must not overconclude the reference to reaction-diffusion systems in the context of field-theory for the effects of internal fluctuations on fronts in general. To make this point clearer, I want to draw the reader's attention to the fact that there exists a vast literature on field-theoretical treatments of the effect of internal fluctuations on fronts in various reaction-diffusion systems, where two opposing currents of reactant species of particles meet from two sides and reactions between them take place in a reasonably localized volume of space known as the reaction zone. In the reaction zone, one can model the fluctuations in particle densities by means of a field-theory, and one obtains various kinds of spatiotemporal behaviour of particle densities or correlations between reacting particles etc (see for example, Refs. [64,65]). In these systems reaction fronts do develop, but they do not propagate (asymptotically) with a constant speed. The dynamics of these fronts are extremely interesting on their own merit, and their understanding has contributed significantly to renormalization group techniques, but as far as this review article is concerned, they are beyond our purview.

On the other hand, as far as internal fluctuations in propagating fronts are concerned, Langevin type field-theories of reaction-diffusion fronts were of interest first to chemists $[67,68]$ and mathematicians [83]. To be more particular, the interest was in a Langevin description of stochastic Fisher equation. It would still be another few years until physicists found field-theories for propagating fronts to be of considerable interest $[35,36,95]$. 
So why are field-theories of internal fluctuations in propagating fronts so difficult? The answer to this question, in fact, lies at two levels. The difficulty at the first level stems from the complication associated with the description of fluctuations in a real experimental situation. In the second paragraph of Sec. 3 , we discussed this briefly using the example of a chemical reaction - that in general, in a chemical reaction, not only are there local fluctuations in the density of reactants, but also complicated correlations between fluctuation in particle densities and in the mesoscopic parameters such as reaction rate or diffusion coefficient of particles exist. Taking all these into account in a reasonable and satisfactory degree of detail is indeed very difficult, and other than Ref. [69] in the chemistry literature, I am not aware of any treatment where the effect of local fluctuations in the mesoscopic reaction-diffusion parameters on the propagating front has been considered.

To get a good theoretical grip on reaction-diffusion processes, just like we have seen all along in Sec. 2, in all the field-theoretical approaches based on Langevin type stochastic differential equations, it is customary to consider fluctuations only in the density of particles while the usual mesoscopic parameters such as diffusion coefficients or reaction rates are held constant. Even then, these field theories are not free from intricacies. A glance at the results (a)-(c) below in the chemistry literature $[67,68,70]$ will make this clear:

(a) For the reaction-diffusion process $\mathrm{X}+\mathrm{Y} \rightarrow 2 \mathrm{X}$ described by the Fisher equation (1) with $n=2$, where the Langevin forces have been obtained from the master equation, a front speed higher than $v^{*}$ has been observed in Ref. [67]. It appears from the results of the numerical simulation that in the range $N \sim 10^{2}-10^{3.5}, v_{N}$ behaves as $v_{N}-v^{*} \sim N^{-1 / 3}$.

(b) In a Ginzburg-Landau or Schlögl model (55), a similar Langevin formalism deduced from the master equation also yields a front speed higher than $v^{\dagger}$ [68]. Numerical simulations in this case yields $v_{N}-v^{\dagger} \sim N^{-1.38}$.

(c) Direct Monte Carlo simulation techniques applied to the reaction-diffusion process $\mathrm{X}+\mathrm{Y} \rightarrow 2 \mathrm{X}$ described by the Fisher equation (1) with $n=2$, however yields a decrease in the front speed from $v^{*}$, and as a function of $N$ in the range $N \sim 10-10^{3.5}, v^{*}-v_{N} \sim N^{-1 / 3}[70]$.

Note that given our prior knowledge of how $v_{N}$ behaves as a function of $N$ from Sec. 2, it is difficult to reconcile (a) and (b) with anything else [even with (c), which in itself is not the asymptotic behaviour of $v_{N}$ for $N \rightarrow \infty$ ]; and this is precisely where the second level of difficulty comes in. The point is that the Langevin type field-theories derived from the master equation effectively are expansions over the "small parameter" $1 /\left\langle\left\langle N_{k}\right\rangle\right\rangle$, where $\left\langle\left\langle N_{k}\right\rangle\right\rangle$ is the conditionally averaged number of particles on lattice site $k$ (see for example Chap. VII of Ref. [52], or Ref. [16]). Clearly, the $1 /\left\langle\left\langle N_{k}\right\rangle\right\rangle$ expansion is only 
possible if $\left\langle\left\langle N_{k}\right\rangle\right\rangle$ are large quantities — so while the expansion is valid in the bulk phase of the front, it break down at the tip of the front, where there are a very few particles per lattice site. At least for a fluctuating "pulled" front such as the stochastic Fisher equation, we already know that its properties are tremendously sensitive to the fluctuation dynamics at the tip of the front. For this reason alone, such Langevin type field theories has to be applied with extreme caution, as we illustrate in the next two paragraphs below.

For fluctuating "pulled" fronts, the reason why the results from the chemists' side $[67,68,70]$ contradict the $1 / \ln ^{2} N$ convergence of the front speed $v_{N}$ to $v^{*}$ from below is not difficult to trace - it lies in the fact that the form of the stochastic terms that they consider amounts to an additive noise to the deterministic equation. If we compare the resulting evolution equation of the front with the microscopic reaction-diffusion process $\mathrm{X} \leftrightharpoons 2 \mathrm{X}$ for example, the contrast between the physical implications of the stochastic differential equation and what actually happens in the microscopic process becomes really clear. In this microscopic process, lattice sites on which there is no particle to start with cannot grow a particle on its own, meaning that the first particle on any lattice site has to diffuse from one of its nearest neighbours. When this idea is applied to the lattice sites that lie ahead of the front, one immediately recovers the idea of the i.f.o.l.s. (see footnote 36). The lattice sites on the right of the i.f.o.l.s. cannot generate particles on their own, and that was the motivation of Brunet and Derrida behind using a growth cutoff for $\phi<1 / N$. The growth cutoff then predicted that the leading edge of the front would be of finite extent, and it also gave rise to the $1 / \ln ^{2} N$ convergence of the front speed $v_{N}$ to $v^{*}$ from below for fluctuating "pulled" fronts.

An additive noise term totally changes this scenario. In that case, any place ahead of the actual front region where $\phi$ value is identically zero at a given time can generate a non-zero $\phi$ within an infinitesimal time interval simply due to fluctuations. These fluctuations then have the capability of pulling the rest of the front faster than even the leading edge of the deterministic pulled fronts! It is therefore no wonder that these models $[67,68,70]$ do yield front speeds that are higher than $v^{*}$.

\subsection{Fluctuating "Pulled" Fronts in Stochastic Fisher-Kolmogorov-Petrovsky- Piscunov (sFKPP) Equation}

From the discussions in the last few paragraphs above, it is clear that as far as a stochastic differential (Fisher-type) equation is concerned, additive noise to model fluctuating "pulled" fronts is out of the question. The noise value has to be zero where the $\phi$ value is zero and this requirement then points the finger to multiplicative noise. 
While this argument is generally valid, question remains regarding what form of multiplicative noise one should choose. Clearly, one should certainly avoid a noise term that behaves $\sim \tilde{\varepsilon}^{1 / 2} \phi$ for $\phi \rightarrow 0-$ for example the case of fluctuating pulled fronts with multiplicative noise as we encountered in Sec. 3. For such noise terms, no matter howsoever small $\phi$ is, the noise amplitude is always an order of $\tilde{\varepsilon} \ll 1$ weaker than $\phi$, and one does not expect such noise terms to yield a leading edge of a finite extent for the front (although I have not seen any work proving or disproving it).

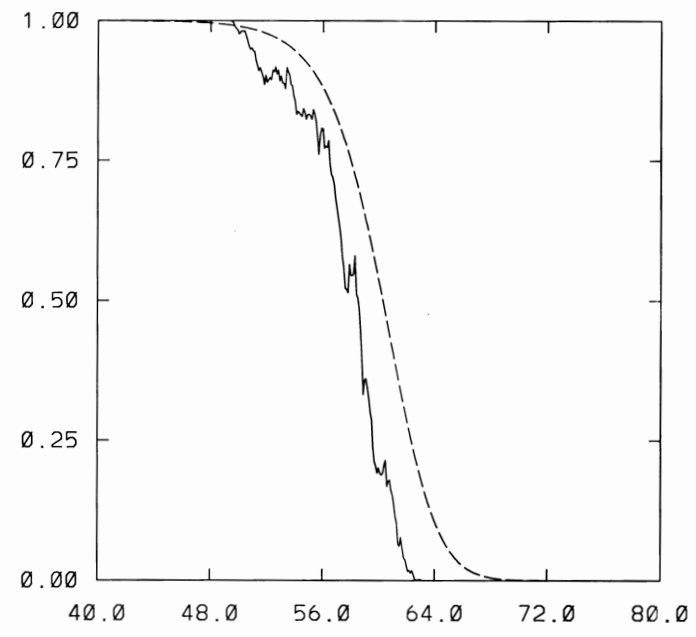

Fig. 35. A snapshot of the fluctuating "pulled" front with $D=1$ in sFKPP equation (solid curve) demonstrating compact support property $[35,36]$. The corresponding plot for the deterministic pulled front in Fisher equation (1) $[n=2]$ is shown with dashed lines for comparison.

It turns out, however, that for the front in Fisher-type (reaction-diffusion) equation, the most frequently used noise term that does yield a leading edge of a finite extent in fact behaves $\sim \tilde{\varepsilon}^{1 / 2} \sqrt{\phi}$ for $\phi \rightarrow 0$ (of this, the $\tilde{\varepsilon} \ll 1$ and $\tilde{\varepsilon} \gg 1$ limits are respectively referred to as the weak noise and the strong noise limits). The corresponding stochastic differential equation is then known as the stochastic Fisher-Kolmogorov-Petrovsky-Piscunov, or in short, the sFKPP equation $[35,36,83,95]$ :

$$
\frac{\partial \phi}{\partial t}=D \frac{\partial^{2} \phi}{\partial x^{2}}+\phi-\phi^{2}+\tilde{\varepsilon}^{1 / 2} \sqrt{\phi-\phi^{2}} \eta(x, t)
$$

Here $\eta(x, t)$ satisfies the properties that $\langle\eta(x, t)\rangle_{\eta}=0$ and $\left\langle\eta(x, t) \eta\left(x^{\prime}, t^{\prime}\right)\right\rangle_{\eta}=$ $2 \delta\left(x-x^{\prime}\right) \delta\left(t-t^{\prime}\right)$. The stochastic term is interpreted in the Itô sense. The factor of 2 in the noise correlation is really unnecessary, but it is introduced to maintain consistency with the corresponding form (65) in Sec. 3.

For the microscopic reaction-diffusion process $\mathrm{X} \leftrightharpoons 2 \mathrm{X}$ however, the sFKPP equation (96) has been derived in terms of a path integral formalism [95], 
which avoids the subtleties associated with very few particles per lattice site at the leading edge of the front. In this derivation, one first rewrites the full master equation in a path integral form, and then proceeds with the idea that the fluctuations in the front around the mean front shape are always small and retains the effects of the fluctuations only at the leading order. The compact support property for sFKPP equation - not only that there exists an $x_{r}(t)$ for which $\phi\left[x>x_{r}(t), t\right]=0$, but also there exists an $x_{l}(t)$ for which $\phi\left[x<x_{l}(t), t\right]=1$, and that $\left[x_{r}(t)-x_{l}(t)\right]<\infty \forall t$ - has been rigorously derived too [83] (see Fig. 35). Although none of these derivations will be discussed here any further, note that the compact support property of the fronts in sFKPP equation is not a surprise. Both around $\phi \sim \tilde{\varepsilon}$ and $\phi \sim 1-\tilde{\varepsilon}^{1 / 2}$, the strengths of the stochastic term are respectively of the same order as $\phi$ and $1-\phi$, and that helps to abruptly cut down the growth of $\phi$ ahead of and the decay of $1-\phi$ behind the front region.

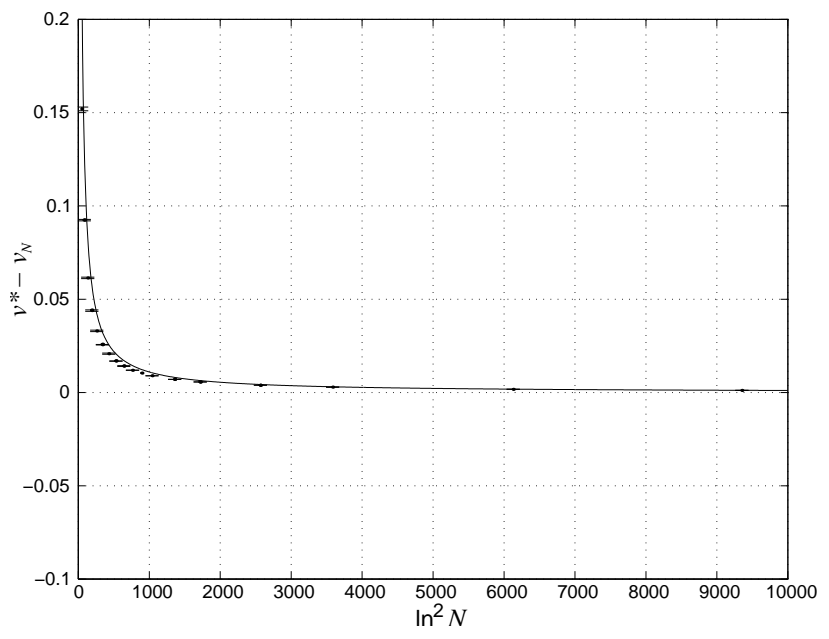

Fig. 36. Speed for the fluctuating "pulled" front in the sFKPP equation on a discrete lattice with $D=1$ and $\tilde{\varepsilon}=1 / 2 N$ [95]. Circles: simulation data (with error bars), solid line: the theoretical expression (11). The symbols have been changed from the original to maintain notational consistency.

In the weak noise limit, $\tilde{\varepsilon}$ is replaced by $1 / 2 N$ (the factor of 2 is put in to counter the 2 appearing in the expression of $\left.\left\langle\eta(x, t) \eta\left(x^{\prime}, t^{\prime}\right)\right\rangle_{\eta}\right)$ for $N \rightarrow \infty$ to make contact with the reaction-diffusion system $\mathrm{X} \leftrightharpoons 2 \mathrm{X}$. Even in this limit, due to the non-local nature of sFKPP equation, it is not possible to obtain a theoretical expression for the speed of the corresponding fluctuating "pulled" front (although some simple local forms of it can be solved exactly at all noise strengths [95]). Nevertheless, one can put it in the computer [95]. For $N \rightarrow \infty$, the speed of the fluctuating "pulled" front is then seen to confirm the $1 / \ln ^{2} N$ convergence (11) of the front speed $v_{N}$ to $v^{*}$ from below (see Fig. 36). 


\subsubsection{Fronts and Duality between the Weak and the Strong Noise Limits in the sFKPP Equation}

The sFKPP equation, in fact, is more versatile than what appears from above. What is required is that one puts it in appropriate perspective.

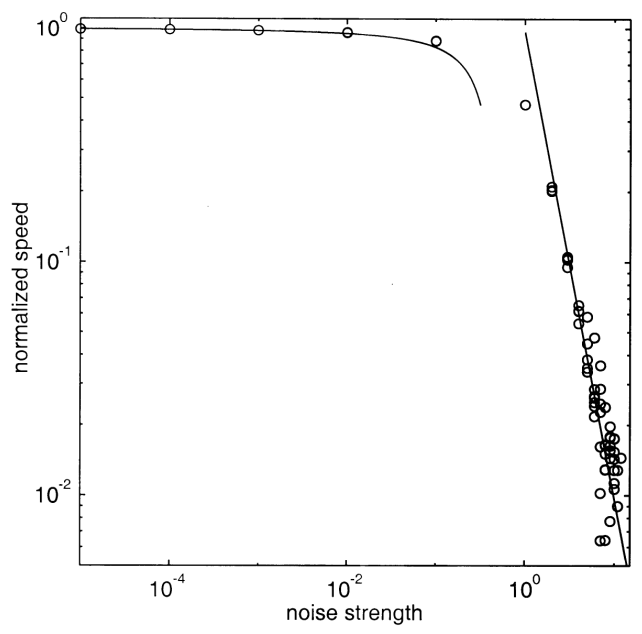

Fig. 37. Log-log plot of $v_{N} / v^{*}$ in the sFKPP equation on a discrete lattice as a function of noise strength $\tilde{\varepsilon}^{1 / 2}[35,36]$. Open circles: simulation data for the sFKPP equation, solid lines: the theoretical expressions (11) at weak noise $\tilde{\varepsilon} \ll 1$ and (97) at strong noise $\tilde{\varepsilon} \gg 1$.

Consider once again the reaction-diffusion process $\mathrm{X} \leftrightharpoons 2 \mathrm{X}$. We had earlier seen, in Secs. 2.3 and 2.6 that $N$, the number of particles per lattice site at the stable phase of the front, dictates the strength of the fluctuations. The smaller $N$ is, the (relatively) stronger the fluctuations become (c.f. Sec. 2.6). On the other hand, since $N$ is essentially the ratio between the rates of the forward and the backward reactions in the $\mathrm{X} \leftrightharpoons 2 \mathrm{X}$ process $[16,35,36,87]$, in Eq. (96), the noise strength $\tilde{\varepsilon} \sim 1 / N$ is decided by this ratio.

The formal connection between the noise strength and the ratio between the forward vs. backward reactions in the reaction-diffusion process $\mathrm{X} \leftrightharpoons 2 \mathrm{X}$ has recently been rigorously proved through the notion of duality $[35,36]$. This connection then enables one to predict the front speed at strong noise $\tilde{\varepsilon} \gg 1$ in the following way: the strong noise limit reduces the $\mathrm{X} \leftrightharpoons 2 \mathrm{X}$ system to the model of Sec. 2.6.5 in the $\varepsilon \rightarrow 0$ and $W \rightarrow \infty$ limit, ${ }^{72}$ while keeping $D \sim \mathcal{O}(1)$. In this limit $N=\varepsilon /(\varepsilon+W) \simeq \varepsilon / W$, and then very easily, Eq. (51) predicts a front speed

\footnotetext{
$\overline{72}$ Although the $\mathrm{X} \leftrightharpoons 2 \mathrm{X}$ system formally allows more than one particles per lattice site, effectively no lattice site ever gets occupied by more than one particles when the backward reaction rate is much higher than the forward reaction rate. The condition for the model of Sec. 2.6.5 that each lattice site can be occupied by at most one particle at any given time is then automatically satisfied.
} 


$$
v_{N}=D N \sim \frac{D}{\tilde{\varepsilon}} .
$$

The rescaled front speed $v_{N} / v^{*}=v_{N} /(2 \sqrt{D \varepsilon})$ is plotted as a function of the noise strength $\tilde{\varepsilon}^{1 / 2}$ in Fig. $37[35,36]$. The open circles denote simulation results for the sFKPP equation and the solid lines denote the corresponding theoretical results for weak [Eq. (11)] and strong [Eq. (97)] noise.

\subsection{The $D_{f}$ and $D_{G}$ Dilemma}

From what we learnt in Sec. 4.1, it seems that the sFKPP equation, interpreted in the Itô sense, does provide a lot a of input to the subject of fluctuating "pulled" fronts - it correctly predicts the front speed behaviour as a function of the noise strength for the limiting cases of both the weak (very large values of $N)$ and strong noise $(N \ll 1)$.

There is, however, a missing piece of the puzzle in the success story of the stochastic differential equations for internal fluctuations, namely that the front diffusion coefficient in one spatial dimension has been explored in neither for the strong and nor for the weak noise. As the issue of front diffusion now becomes our focus, at the very first step, it becomes a necessity that for general reaction-diffusion systems, we reconcile $D_{f}$ of Sec. 2.5 and $D_{G}$ of Sec. 3 , both introduced for the measure of front diffusion.

\subsection{1 $D_{f}$ vs. $D_{G}$ : What Exactly is the Difference?}

We start with the Itô stochastic differential equation for $\tilde{\varepsilon} \ll 1$

$$
\frac{\partial \phi}{\partial t}=D \frac{\partial^{2} \phi}{\partial x^{2}}+f(\phi)+\tilde{\varepsilon}^{1 / 2} R[\phi(x, t), x, t]
$$

[which is of the same form as Eq. (73)]. Here $R[\phi(x, t), x, t]=g[\phi(x, t)] \eta(x, t)$, and in the Itô interpretation,

$$
\begin{aligned}
& \langle R[\phi(x, t), x, t]\rangle_{\eta}=g[\phi(x, t)]\langle\eta(x, t)\rangle_{\eta}=0 \quad \text { and } \\
& \begin{aligned}
\left\langle R[\phi(x, t), x, t] R\left[\phi\left(x^{\prime}, t^{\prime}\right), x^{\prime}, t^{\prime}\right]\right\rangle_{\eta} & =g[\phi(x, t)] g\left[\phi\left(x^{\prime}, t^{\prime}\right)\right]\left\langle\eta(x, t) \eta\left(x^{\prime}, t^{\prime}\right)\right\rangle_{\eta} \\
& =2 g^{2}[\phi(x, t)] \delta\left(x-x^{\prime}\right) \delta\left(t-t^{\prime}\right) .
\end{aligned}
\end{aligned}
$$

In Eq. (98), $D_{G}$ is defined from $\dot{X}(t)$, the random displacement of the Goldstone mode of the front [see Eq. (80)]. On the other hand, if one measures the position of the front by its centre of mass location using the continuum 
version of Eq. (24), then from Eq. (78) one obtains, for the fluctuation in the speed of the centre of mass of the front,

$$
\dot{S}(t)=\dot{X}(t)+\int_{-\infty}^{\infty} d \xi \delta \dot{\phi}(\xi, t)
$$

where $\delta \dot{\phi}(t)$ is measured on the instantaneous comoving frame of the Goldstone mode.

Next, having simplified $\dot{S}(t)$ with the aid of Eqs. (77) and (79), and with the inherent assumption that $\delta \phi(\xi, t) \rightarrow 0$ for $\xi \rightarrow \pm \infty$ as

$$
\dot{S}(t)=\left.\int_{-\infty}^{\infty} d \xi \frac{\delta f(\phi)}{\delta \phi}\right|_{\phi^{(0)}} \delta \phi(\xi, t)+\tilde{\varepsilon}^{1 / 2} \int_{-\infty}^{\infty} d \xi R[\phi(\xi, t), \xi, t]
$$

we immediately notice something interesting — namely that through Eq. (101), we have essentially recovered Eq. (27). Remember that Eq. (27) was obtained through a heuristic argument for the clock model! For the uniformly translating front solution $\phi^{(0)}$ of the deterministic reaction-diffusion equation $\partial_{t} \phi=D \partial_{x}^{2} \phi+f(\phi)$, the front speed is given by $\int_{-\infty}^{\infty} d x f\left[\phi^{(0)}\right]$, and naturally, when the distribution of $\phi(x, t)$ deviates from $\phi^{(0)}$, at the leading order, the deviation in the front speed is given by $\left.\int_{-\infty}^{\infty} d x \frac{\delta f(\phi)}{\delta \phi}\right|_{\phi^{(0)}} \delta \phi(x, t)$ just like we had seen in Eq. (26). Secondly, in Eq. (101), we have not replaced $R[\phi(\xi, t), \xi, t]$ by $R\left[\phi^{(0)}(\xi, t), \xi, t\right]$ as we did in Sec. 3. In fact, the reason we had to do so in Sec. 3 is to make the stochastic term in Eq. (72) $\delta$-correlated both in space and time in the Stratonovich interpretation. In the Itô interpretation (99) this replacement is no longer necessary. The choice of not replacing $R[\phi(\xi, t), \xi, t]$ by $R\left[\phi^{(0)}(\xi, t), \xi, t\right]$, however, implies that to obtain the front diffusion coefficient, we would first have to average over the noise realizations (subscript $\eta$ ) and then average over an ensemble of initial front realizations at time $t$ (subscript $t$ ), just like in Eq. (28):

$$
D_{f}=\frac{1}{2} \lim _{T \rightarrow \infty} \int_{0}^{T} d t^{\prime}\left\langle\left\langle\dot{S}(t) \dot{S}\left(t+t^{\prime}\right)\right\rangle_{\eta}\right\rangle_{t}
$$

Thirdly, with $\langle R[\phi(\xi, t), \xi, t]\rangle_{\eta}=0$, the average of Eq. (101) over all possible noise realizations at time $t$ does not yield $\langle\dot{S}(t)\rangle_{\eta}=0$. Indeed, this is the reason why we had a non-zero $\delta v_{\mathrm{r}, \mathrm{mf}}(t)$ in Eq. (25). Only when a further average over 
all possible front realizations at time $t$ is taken, by virtue of $\langle\delta \phi(\xi, t)\rangle_{t}=0$, one gets $\langle\dot{S}(t)\rangle=0$. This is also the same as in Eq. (27).

Now to simplify Eq. (102). The r.h.s. of Eq. (101) contains a sum of two terms, and the product of $\dot{S}(t) \dot{S}\left(t+t^{\prime}\right)$ that appears in the Green-Kubo formula (102) contains four terms. Of these, an average over all possible noise realizations after time $t$ kills the $\left.\int_{-\infty}^{\infty} d \xi \frac{\delta f(\phi)}{\delta \phi}\right|_{\phi^{(0)}} \delta \phi(\xi, t) \int_{-\infty}^{\infty} d \xi^{\prime} R\left[\phi\left(\xi^{\prime}, t+t^{\prime}\right), \xi^{\prime}, t+t^{\prime}\right]$ but the rest three survive. Among these three surviving terms, the easiest one to handle is $\int_{-\infty}^{\infty} d \xi R[\phi(\xi, t), \xi, t] \int_{-\infty}^{\infty} d \xi R\left[\phi\left(\xi, t+t^{\prime}\right), \xi, t+t^{\prime}\right]$. With Eq. (99), its contribution to $D_{f}$ is seen to be

$$
D_{f}^{(1)}=\tilde{\varepsilon} \int_{-\infty}^{\infty} d \xi\left\langle g^{2}[\phi(\xi, t)]\right\rangle_{t}
$$

To obtain the contribution $D_{f}^{(2)}$ of $\left.\int_{-\infty}^{\infty} d \xi R[\phi(\xi, t), \xi, t] \int_{-\infty}^{\infty} d \xi^{\prime} \frac{\delta f(\phi)}{\delta \phi}\right|_{\phi^{(0)}} \delta \phi\left(\xi^{\prime}, t+t^{\prime}\right)$, we formally solve $\delta \phi\left(\xi, t+t^{\prime}\right)$ first by expanding it as in Eq. (82), and then by using Eq. (85) (without having replaced $R\left[\phi\left(\xi, t^{\prime \prime}\right), \xi, t^{\prime \prime}\right]$ by $R\left[\phi^{(0)}\left(\xi, t^{\prime \prime}\right), \xi, t^{\prime \prime}\right]$ ). Thereafter, Eq. (99) and the integration over $t^{\prime}$ yield

$$
D_{f}^{(2)}=\left.\tilde{\varepsilon} \int_{-\infty}^{\infty} d \xi \int_{-\infty}^{\infty} d \xi^{\prime} \sum_{m \neq 0}\left[\tau_{m} \Psi_{m, L}(\xi) \Psi_{m, R}\left(\xi^{\prime}\right)\right]\left\langle g^{2}[\phi(\xi, t)]\right\rangle_{t} \frac{\delta f(\phi)}{\delta \phi}\right|_{\phi^{(0)}\left(\xi^{\prime}\right)}
$$

This is still not simple enough. To reduce Eq. (104) further, we return to Eqs. (16) and (17) and use the fact that $\left.\int_{-\infty}^{\infty} d \xi^{\prime} \Psi_{m, R}\left(\xi^{\prime}\right) \frac{\delta f(\phi)}{\delta \phi}\right|_{\phi^{(0)}\left(\xi^{\prime}\right)} ^{=} \tau_{m}^{-1} \int_{-\infty}^{\infty} d \xi^{\prime} \Psi_{m, R}\left(\xi^{\prime}\right)$. With the completeness relation (86), we then get a simple expression:

$$
D_{f}^{(2)}=\tilde{\varepsilon} \int_{-\infty}^{\infty} d \xi\left\langle g^{2}[\phi(\xi, t)]\right\rangle_{t}
$$

Finally, proceeding exactly along the lines of Eqs. (104) and (105), $D_{f}^{(3)}$, the contribution of the $\left.\left.\int_{-\infty}^{\infty} d \xi \frac{\delta f(\phi)}{\delta \phi}\right|_{\phi^{(0)}} \delta \phi(\xi, t) \int_{-\infty}^{\infty} d \xi^{\prime} \frac{\delta f(\phi)}{\delta \phi}\right|_{\phi^{(0)}} \delta \phi\left(\xi^{\prime}, t+t^{\prime}\right)$ term to $D_{f}$ can also be simplified to 


$$
D_{f}^{(3)}=\sum_{m, m^{\prime} \neq 0} \tau_{m}^{-1}\left\langle c_{m}(t) c_{m^{\prime}}(t)\right\rangle_{t} \int_{-\infty}^{\infty} d \xi \int_{-\infty}^{\infty} d \xi^{\prime} \Psi_{m, R}(\xi) \Psi_{m^{\prime}, R}\left(\xi^{\prime}\right)
$$

To obtain $D_{f}=D_{f}^{(1)}+D_{f}^{(2)}+D_{f}^{(3)}$, one now has to calculate the average of the quantities \langle\rangle$_{t}$ in Eqs. (103), (105) and (106), i.e., over an ensemble of (initial) realizations at time $t$. When $R[\phi(\xi, t), \xi, t]$ is replaced by $R\left[\phi^{(0)}(\xi, t), \xi, t\right]$ in the Stratonovich interpretation of Sec. 3, this is an easy task: while $\int_{-\infty}^{\infty} d \xi g^{2}\left[\phi^{(0)}(\xi)\right]$ is very easy to evaluate directly, Eq. (88) effectively becomes a Langevin equation (with additive noise) for a Brownian particle in a viscous fluid. One can then simply use fluctuation-dissipation theorem to evaluate $\left\langle c_{m}(t) c_{m^{\prime}}(t)\right\rangle_{t}$. In the Itô interpretation, however, one does not require to replace $R[\phi(\xi, t), \xi, t]$ by $R\left[\phi^{(0)}(\xi, t), \xi, t\right]$ anywhere between Eqs. (100) and (106), and then strictly speaking, one cannot use the fluctuationdissipation theorem for multiplicative noise to evaluate $\left\langle c_{m}(t) c_{m^{\prime}}(t)\right\rangle_{t}$ from Eq. (88) any longer - is is a well-known problem in Stochastic processes that there is no fluctuation-dissipation theorem for multiplicative noise (see, e.g., Ref. [52]).

Nevertheless, what is the problem if we simple-mindedly replace $R[\phi(\xi, t), \xi, t]$ by $R\left[\phi^{(0)}(\xi, t), \xi, t\right]$ ? It certainly makes calculations easier. As for the answer to this question, we note that one really has to be very careful to do this. The integrand in Eq. (87) contains $\Psi_{m, L}(\xi)=e^{v_{\varepsilon} \xi /(2 D)} \psi_{m}(\xi)$, and due to the presence of the exponential weight factor $e^{v_{\varepsilon} \xi /(2 D)}$, it is the fluctuations at the tip of the front that matter the most. This further implies that $c_{m}$ 's are strong functions of time in general - after all, in case of internal fluctuations of fronts, the fluctuations at the tip of the front are of the order of the front value itself. ${ }^{73}$ However, when the nonlinearity $f(\phi)$ makes the front pushed, i.e., for fluctuating pushed fronts, all fluctuation modes have a finite lifetime comparable to the time scale set by $1 / v_{\varepsilon}$. In that case, the dependence of the precise value of $\delta \phi$ in one snapshot of a front realization at any time $t$ at the tip of the front does not matter so much on the precise noise realization that has been used to update the front profile before $t$. This then justifies the replacement of $R[\phi(\xi, t), \xi, t]$ by $R\left[\phi^{(0)}(\xi, t), \xi, t\right]$ [and further on the usage of fluctuation-dissipation theorem to obtain $\left.\left\langle c_{m}(t) c_{m^{\prime}}(t)\right\rangle_{t} \sim \mathcal{O}(\tilde{\varepsilon})\right]$. For fluctuating "pulled" fronts however, such as the sFKPP equation the fluctuation modes can have very long decay times (c.f. Sec. 2.4.2). Then the the replacement of $R[\phi(\xi, t), \xi, t]$ by $R\left[\phi^{(0)}(\xi, t), \xi, t\right]$ and the subsequent usage of fluctuation-dissipation theorem to obtain $\left\langle c_{m}(t) c_{m^{\prime}}(t)\right\rangle_{t}$ is simply incorrect!

$\overline{73}$ This actually depends on the functional form of $g(\phi)$. For example, when $g(\phi) \sim$ $\sqrt{\phi}$ for $\phi \rightarrow 0$, the fluctuations at the tip of the front are certainly as strong as $\phi$. 
For fluctuating pushed fronts, when $R[\phi(\xi, t), \xi, t]$ is replaced everywhere by $R\left[\phi^{(0)}(\xi, t), \xi, t\right]$, one can easily show that $D_{f}^{(1)}=D_{f}^{(2)}=2 D_{f}^{(3)}$ [after using fluctuation-dissipation theorem in Eq. (84) and thereafter with the completeness relation (86)], yielding $D_{f}=5 \tilde{\varepsilon} \int_{-\infty}^{\infty} d \xi g^{2}\left[\phi^{(0)}(\xi)\right] / 2$. This expression is clearly different from that of $D_{G}$ in Eq. (81). ${ }^{74}$ As for fluctuating "pulled" fronts, the scalings of $D_{f}$ and $D_{G}$ will now be dealt with in Sec. 4.2.2.

\subsection{2 $D_{f}$ and $D_{G}$ for the sFKPP Equation}

From the point of view that the sFKPP equation belongs to the family of Eq. (98), a question that naturally arises is whether it is possible to apply our collected wisdom of Sec. 4.2.1 to the sFKPP equation.

A priori, one can expect such an attempt to fail, or at best expect it to be full of subtleties, for by now, we have already learnt from Sec. 3.3 that in the limit of zero noise strength, whenever Eq. (98) gives rise to a pulled front, $D_{G}$ [Eq. (81)] does not remain well defined any more. This simply has to do with the fact that the integral in the denominator of Eq. (81) diverges due to the semi-infinite leading edge of pulled fronts.

We can however ask ourselves the following question: from Brunet and Derrida's analysis, we know that an effective description of fluctuating "pulled" fronts in Fisher equation is in terms of a growth cutoff below $\phi \simeq 1 / N$. So how about using Brunet and Derrida's cutoff solution for $\phi^{(0)}$ in the whole formalism of Sec. 4.2.1, assuming that the shape fluctuation of the front in the sFKPP equation are indeed described by the shape fluctuation modes $\left\{\Psi_{m}\right\}{ }^{75}$ At least in that case, any complication associated with the divergence of the denominator Eq. (81) is safely avoided!

What we will show in this section is that such an simple-minded approach works [91]. It yields the asymptotic scalings $D_{G} \sim 1 / \ln ^{6} N$ and $D_{f} \sim 1 / \ln ^{3} N$. Although at the time of writing this review article, neither $D_{G}$ nor $D_{f}$ for the sFKPP equation has been studied (analytically or numerically), we will later argue that the asymptotic scaling $D_{f} \sim 1 / \ln ^{3} N$ is a generic property of

\footnotetext{
${ }^{74}$ For fluctuating pushed fronts made of discrete particles on a lattice, $\tilde{\varepsilon} \sim 1 / N$. This indicates that for these lattice models, one does obtain a $D_{f}$ that scales asymptotically as $1 / N$. From Eq. (81), $D_{G}$ too is seen to scale as $1 / N$.

${ }^{75}$ Recall that in this case, $\left\{\Psi_{m}\right\}$ are localized within the front region and they vanish for $\xi \rightarrow \pm \infty$ (c.f. Sec. 2.4.2). This is precisely the compact support property of the front solution in the sFKPP equation entails; and moreover, it is the same requirement that underlies Eq. (100). This means that all the results we obtained in Sec. 4.2.1 can be safely applied to this case.
} 
fluctuating "pulled" fronts.

Between $D_{G}$ and $D_{f}$, the $1 / \ln ^{6} N$ asymptotic scaling for $D_{G}$ is the easiest to demonstrate. For simplicity, we set $D=1$. With $\tilde{\varepsilon}=1 /(2 N)$ and $g(\phi)=$ $\sqrt{\phi-\phi^{2}}$ for the sFKPP equation, from Eq. (81). we obtain

$$
D_{G}=\left[\int_{-\infty}^{\xi_{c}} d \xi e^{2 v_{N} \xi}\left(\frac{d \phi^{(0)}}{d \xi}\right)^{2} \frac{\left\langle\phi(\xi, t)-\phi^{2}(\xi, t)\right\rangle_{t}}{2 N}\right] /\left[\int_{-\infty}^{\xi_{c}} d \xi e^{v_{N} \xi}\left(\frac{d \phi^{(0)}}{d \xi}\right)^{2}\right]^{2} \cdot(107
$$

When one uses Eq. (10) $[\varepsilon=1 / N]$ in Eq. (107), the exponential weight factor in the integrand of the denominator drops out and the denominator itself asymptotically scales as $1 / \ln ^{6} N$ at the leading order. Dealing with the integrand of the numerator, however, is slightly more complicated - there exists a factor of $e^{v_{N} \xi}$ in the expanded expression of $e^{2 v_{N} \xi}\left(\frac{d \phi^{(0)}}{d \xi}\right)^{2} \cdot{ }^{76}$ The presence of this exponential weight factor in the integrand means that mathematically, we need to consider $\left\langle\phi(\xi, t)-\phi^{2}(\xi, t)\right\rangle_{t}$ pretty much only at the tip of the front (i.e., around $\xi_{0}$, where the cutoff is implemented; see Fig. 13), and physically it means that the fluctuations at the tip of the front contribute the most to $D_{G}$. At this region, due to the fact $\phi^{2}(\xi, t) \ll \phi(\xi, t), \phi^{2}(\xi, t)$ can be dropped from Eq. (107). Thereafter, with $\langle\phi(\xi, t)\rangle_{t}=\phi^{(0)}(\xi)$, the contribution of the tip region to the numerator turns out to be of $\mathcal{O}(N)$ at the leading order, which then cancels the $1 / N$ prefactor.

The $1 / \ln ^{6} N$ asymptotic scaling of $D_{G}$ is finally obtained by noticing that the region behind the tip cannot contribute enough to cancel the $1 / N$ prefactor.

The $1 / \ln ^{3} N$ asymptotic scaling of $D_{f}$, on the other hand, is a much more involved process; but we can already considerably simplify its expression with the results of Sec. 4.2.1. For example, $D_{f}=D_{f}^{(1)}+D_{f}^{(2)}+D_{f}^{(3)}$ is a simplification. We also know that Eqs. (103), (105) and (106) are exact results for the Ito differential equation (98). Since $D_{f}^{(1)}$ and $D_{f}^{(2)}$ for the sFKPP equation are easily seen to scale $\sim 1 / N$ from Eqs. (103) and (105), our focus naturally concentrates on the asymptotic scaling of $D_{f}^{(3)}$.

Formally, it is not possible to simplify Eq. (106) any further. We have also demonstrated in Sec. 4.2.1 that one cannot also simply replace $\phi(\xi, t)$ by $\phi^{(0)}(\xi)$ everywhere for fluctuating "pulled" fronts — doing so yields the wrong form $D_{f}^{(3)}$, namely that $D_{f}^{(3)}=D_{f}^{(1)} \sim 1 / N$. Instead, to evaluate $D_{f}^{(3)}$, one really has to compute the integrals and the sums in Eq. (106). As it turns out, doing so is full of subtleties as well.

\footnotetext{
${ }^{76}$ We are using, at the leading order, $v_{N} \simeq v^{*}=2=2 \lambda^{*}$.
} 
The first difficulty we face is that we cannot calculate $\int_{-\infty}^{\infty} d \xi \Psi_{m, R}(\xi)$ easily. While we do know the solutions of $\Psi_{m, R}(\xi)$ for the low lying modes from Sec. 2.4 .2 - essentially all the fluctuation modes are localized within the leading edge of the front - there is a danger in using them to evaluate $\int_{-\infty}^{\infty} d \xi \Psi_{m, R}(\xi)$ right away. This is easily demonstrated as follows: at the leading edge, $\Psi_{m, R}(\xi)=A_{m} \sin \left[q_{m}\left(\xi-\xi_{1}\right)\right] e^{-\lambda^{*} \xi}$, where $q_{m}=(m+$ 1) $\pi / \ln N$ and the normalization $A_{m} \simeq \sqrt{2} / \ln ^{1 / 2} N$ is chosen in such a way that $\int_{-\infty}^{\infty} d \xi \Psi_{m, L}(\xi) \Psi_{m^{\prime}, R}(\xi)=\delta_{m, m^{\prime}}$. When this expression of $\Psi_{m, R}(\xi)$ is used for not very high values of $m$, one gets $\int_{-\infty}^{\infty} d \xi \Psi_{m, R}(\xi) \sim(m+1) / \ln ^{3 / 2} N$ at the leading order. The point to note here however, is that since $\lambda^{*}=1$, practically the only contribution to the integral comes from the region around $\xi_{1}$, i.e., at the very left of the leading edge within a distance of $\mathcal{O}\left(1 / \lambda^{*}\right)=\mathcal{O}(1)$, where the argument of the sin-function approaches zero [this is what allowed us to replace $\sin \left[q_{m}\left(\xi-\xi_{1}\right)\right]$ by $\left.q_{m}\left(\xi-\xi_{1}\right)\right]$. Precisely at this region, the nonlinear term in $f(\phi)$, too, becomes significant within a distance of $\mathcal{O}(1)$ (see Fig. 13). Therefore, although in Fig. $13, V(\xi)$ can be replaced by $V_{0}(\xi)$ to obtain $\tau_{m}, \Psi_{m, R}(\xi)$, and the normalization constant $A_{m}$ with a reasonable accuracy, $\Psi_{m, R}(\xi)$ cannot be used to evaluate $\int_{-\infty}^{\infty} d \xi \Psi_{m, R}(\xi)$ so simple-mindedly.

To circumvent this difficulty, we rewrite $D_{f}^{(3)}$ in a different way:

$$
\begin{aligned}
D_{f}^{(3)} & =\left.\frac{1}{2} \lim _{T \rightarrow \infty} \int_{t}^{t+T} d t^{\prime} \int_{-\infty}^{\infty} d \xi \int_{-\infty}^{\infty} d \xi^{\prime}\left\langle\left\langle\left.\left.\frac{\delta f(\phi)}{\delta \phi}\right|_{\phi^{(0)}(\xi)} ^{\delta \phi}(\xi, t) \frac{\delta f(\phi)}{\delta \phi}\right|_{\phi^{(0)}\left(\xi^{\prime}\right)} \underset{-\infty}{\delta}\left(\xi^{\prime}, t+t^{\prime}\right)\right\rangle\right\rangle_{\eta}\right|_{t} \\
& =\left.\left.\sum_{m, m^{\prime} \neq 0} \frac{\tau_{m}\left\langle c_{m}(t) c_{m^{\prime}}(t)\right\rangle_{t}}{2} \int_{-\infty}^{\infty} d \xi \frac{\delta f(\phi)}{\delta \phi}\right|_{\phi^{(0)}(\xi)} ^{\Psi_{m, R}(\xi)} \underset{-\infty}{\infty} d \xi^{\prime} \frac{\delta f(\phi)}{\delta \phi}\right|_{\phi^{(0)}\left(\xi^{\prime}\right)} ^{\Psi_{m^{\prime}, R}\left(\xi^{\prime}\right) . \quad(108)}
\end{aligned}
$$

In this form, $\left.\int_{-\infty}^{\infty} d \xi \frac{\delta f(\phi)}{\delta \phi}\right|_{\phi^{(0)}(\xi)} \underset{m, R}{ }(\xi)$ is the contribution of the $m$-th shape fluctuation mode to $\dot{S}$ [Eq. (101)]. The rationale behind writing $D_{f}^{(3)}$ in this form is to articulate the fact that for the uniformly translating front solution $\phi^{(0)}$, the front speed is given by $\int_{-\infty}^{\infty} d \xi f\left[\phi^{(0)}(\xi)\right]$, and naturally, when the front shape $\phi(x, t)$ deviates from $\phi^{(0)}$ by an amount $\Psi_{m, R}(\xi)$, the contribution of 
the shape fluctuation to the to the fluctuation in the front speed is given by $\delta v_{\mathrm{mf}, m}=\left.\int_{-\infty}^{\infty} d \xi \frac{\delta f(\phi)}{\delta \phi}\right|_{\phi^{(0)}(\xi)} \Psi_{m, R}(\xi)$ at the leading order [just like in Eq. (26) for the clock model; the notation $\delta v_{\mathrm{mf}, m}$, too, is motivated by Eq. (26)].

From the paragraph above Eq. (108), we learnt that to evaluate $\delta v_{\mathrm{mf}, m}$ exactly, we need the full solution of Eq. (17) [this we do not have]. Nevertheless, what we are really interested is how $\delta v_{\mathrm{mf}, m}$ scales with $\ln N$. To determine this, using $A_{m}=\sqrt{2} / \ln ^{1 / 2} N$, we first rewrite the front shape only due to the shape fluctuation mode $\Psi_{m, R}$ at the (linearized) leading edge as

$$
\begin{aligned}
\phi(\xi, t) & =\phi^{(0)}(\xi)+\Psi_{m, R}(\xi) \\
& =\frac{\ln N}{\pi} e^{-\lambda^{*} \xi}\left\{\sin \left[q_{0} \xi\right]+\sqrt{2} \pi \frac{\sin \left[q_{m} \xi\right]}{\ln ^{3 / 2} N}\right\} .
\end{aligned}
$$

In this form, it becomes immediately clear that for any pure normalized fluctuation mode, the fluctuations in the front field are always an order $1 / \ln ^{3 / 2} N$ weaker than the front field itself. Thereafter, as we further realize that for Brunet and Derrida's cutoff solution, $\int_{-\infty}^{\infty} d \xi f\left[\phi^{(0)}(\xi)\right]=v_{N} \simeq v^{*}=2$ is actually of $\mathcal{O}(1)$, we conclude that the presence of the pure normalized $m$-th shape fluctuation mode introduces a fluctuation $\delta v_{\mathrm{mf}, m}$ in the speed of the centre of mass of the front, such that

$$
\delta v_{\mathrm{mf}, m}=\frac{s_{m}}{\ln ^{3 / 2} N}
$$

at the leading order, and $s_{m}$ of $\mathcal{O}(1)$. Equations (108-110) then imply

$$
D_{f}^{(3)}=\frac{1}{2} \sum_{m, m^{\prime} \neq 0} \tau_{m} \frac{s_{m} s_{m^{\prime}}}{\ln ^{3} N}\left\langle c_{m}(t) c_{m^{\prime}}(t)\right\rangle_{t}
$$

Furthermore, since there are $\mathcal{O}(\ln N)$ number of bound states within the potential well $V_{0}(\xi)$ of width $\ln N$ and depth $\mathcal{O}(1)$ [see Fig. 13], the sum over $m$ and $m^{\prime}$ in Eq. (111) runs from 1 to $\ln N$.

The last tricky part now is how to obtain the dependence of $\left\langle c_{m}(t) c_{m^{\prime}}(t)\right\rangle_{t}$ on $\ln N$, and how to evaluate the sums in Eq. (111). To this end, notice that for a given realization, $c_{m}(t)$ is obtained from Eq. (87), and therein, the presence of the factor $e^{\lambda^{*} \xi}$ in the $\Psi_{m, L}(\xi)$ implies that $c_{m}(t)$ for a given realization is practically determined from the fluctuation characteristics at the tip of the front. Thus, in Eq. (87), we keep the integral over $\xi$ only over the leading edge of the front and write 


$$
c_{m}(t)=\frac{1}{\ln ^{1 / 2} N} \int_{\xi_{1}}^{\xi_{0}} d \xi e^{\lambda^{*} \xi} \sin \left[q_{m}\left(\xi-\xi_{1}\right)\right] \delta \phi(\xi, t) .
$$

Although Eq. (112) yields $\left\langle c_{m}(t)\right\rangle_{t}=0$ as it should, in the absence of any statistics of the shape fluctuations of the front, one cannot obtain an expression of $\left\langle c_{m}(t) c_{m^{\prime}}(t)\right\rangle_{t}$ from it. Therefore, as far as any formal derivation of the $1 / \ln ^{3} N$ asymptotic scaling of $D_{f}^{(3)}$ (or $D_{f}$ ) is concerned, one simply cannot progress beyond Eq. (112).

Nevertheless, we can still proceed with two approximations. The first one stems from the fact that although it is clear from Eq. (112) that $c_{m}(t)$ and $c_{m^{\prime}}(t)\left(m \neq m^{\prime}\right)$ are correlated in general [after all, for a given realization, all the $c_{m}(t)$ 's are determined through the same $\left.\delta \phi(t)\right]$, these fluctuation modes will have a finite correlation "length", i.e., $\left\langle c_{m}(t) c_{m^{\prime}}(t)\right\rangle_{t}$ will be negligibly small [compared to both $\left\langle c_{m}^{2}(t)\right\rangle_{t}$ and $\left\langle c_{m^{\prime}}^{2}(t)\right\rangle_{t}$ values] when $\left|m-m^{\prime}\right|$ exceeds a certain threshold $a \ll \ln N$. Based on this observation, our approximation is to choose $a=0$ for the extreme (and unrealistic) case to simplify the expression for $D_{f}^{(3)}$ to (see later for the discussion on non-zero values of $a$ )

$$
D_{f}^{(3)}=\frac{1}{2} \sum_{m \neq 0}^{\ln N} \tau_{m} \frac{s_{m}^{2}}{\ln ^{3} N}\left\langle c_{m}^{2}(t)\right\rangle_{t}
$$

Then the second approximation is that due to the presence of the $e^{\lambda^{*} \xi}$ in the integrand of Eq. (112), only the value of $\delta \phi$ within a distance $\sim 1 / \lambda^{*}=1$ of the tip determines $c_{m}(t)$. This is argued in the following manner: typically the magnitude of $\delta \phi(\xi, t)$ is of order $\sqrt{\phi(\xi, t) / N}$; at the tip, $e^{\lambda^{*} \xi_{0}} \sim N$ cancels $\delta \phi(\xi, t) \sim 1 / N$, but further behind, the $1 / \sqrt{N}$ factor of $\sqrt{\phi(\xi, t) / N}$ can no longer be compensated by $e^{\lambda^{*} \xi}$. We therefore use

$$
\left|c_{m}(t)\right| \sim \frac{1}{\ln ^{1 / 2} N} \int_{\xi_{0}-1}^{\xi_{0}} d \xi\left|\sin \left[q_{m}\left(\xi-\xi_{1}\right)\right]\right| \sim \frac{q_{m}}{\ln ^{1 / 2} N}=\frac{(m+1) \pi}{\ln ^{3 / 2} N}
$$

With $\tau_{m}=\ln ^{2} N /\left[\pi^{2}\left\{(m+1)^{2}-1\right\}\right]$, the asymptotic scaling of $D_{f}$ is finally obtained as

$$
D_{f} \simeq D_{f}^{(3)} \sim \frac{1}{2} \sum_{m \neq 0}^{\ln N} \frac{(m+1)^{2} s_{m}^{2}}{\left[(m+1)^{2}-1\right] \ln ^{4} N} \sim \frac{1}{\ln ^{3} N}
$$

We end Sec. 4.2.2 with four observations: (i) with more reasonable assumption $a \neq 0$, the expression for $D_{f}^{(3)}$ certainly gets more complicated. However, so 
long as $a \ll \ln N$, which is what one expects in reality, the $1 / \ln ^{3} N$ asymptotic scaling of $D_{f}^{(3)}$ (and hence of $D_{f}$ too) still continues to hold. (ii) We have extensively used the construction for the left eigenvector of the stability operator $\mathcal{L}_{v_{N}}$ for Fisher equation (with a growth cutoff below $\phi=1 / N$ ) all along Sec. 4.2.2. For clock model [122-126], or for the model that Brunet and Derrida considered [21] to demonstrate the $1 / \ln ^{3} N$ asymptotic scaling of $D_{f}$, construction of the left eigenvector for the corresponding stability operator is not easy. In view of that I do not know how to repeat the same derivation for those two models with an equivalent amount of rigour. Nevertheless, since all the arguments for the sFKPP equation between Eqs. (109) and (115) are concentrated on the leading edge or at the very tip of the front, they can be easily repeated for these two models to derive the same $1 / \ln ^{3} N$ asymptotic scaling of $D_{f}$. (iii) In the clock model, in terms of $\phi$, one cannot create a localized fluctuation in the front shape - any fluctuation in the front shape is by definition non-local. The "collisions" between clocks are also non-local in nature [as reflected in Eq. (25)]. From these complications, although a priori it may seem that the clock model may have a different asymptotic scaling of $D_{f}$, in reality, it does not happen. In fact, Brunet and Derrida showed in a simplified version of their original microscopic model (which closely resembles the clock model) that the introduction of localized fluctuations in $\phi$ at the very tip of the front is all that is needed for the $1 / \ln ^{3} N$ asymptotic scaling of $D_{f}$. In that sense [also in view of point (ii)] the $1 / \ln ^{3} N$ asymptotic scaling of $D_{f}$ seems to be a generic property of fluctuating "pulled" fronts, independent of the microscopic model. (iv) Last but not the least, through the scaling of the $c_{m}(t)$ 's, the $1 / \ln ^{3} N$ asymptotic scaling of $D_{f}$ is seen to be determined only from the tip of the front. This is in perfect agreement with Brunet and Derrida's "simplified model" [21].

\subsection{Epilogue III}

The existence of two different expressions for front diffusion, $D_{f}$ and $D_{G}$ seems confusing to say the least - after all, any front realization maintains its integrity at all times, and thus, at no instant of time, the Goldstone mode and the centre of mass of any given front realization can be infinitely far apart from each other. This implies that whether measured through the Goldstone mode, the center of mass, or the any place where the value of the front field reaches any fixed value, say $\phi_{0}$, operationally (i.e., in a computer simulation) one should always obtain the same front diffusion coefficient!

In fact, the present situation can be compared to the a similar one that occurs in gas mixtures (see for example, Chap. 11.2 of Ref. [46]) Therein, the expression (and the value) of the the diffusion coefficient depends on its precise definition (although operationally there is exactly one diffusion coefficient), but 
these different expressions of the diffusion coefficient are (quite non-trivially) related by means of the Onsager relations for the diffusion coefficients. As for fronts too, it should not be surprising that the precise values of $D_{f}$ and $D_{G}$ are not the same - conceptually they are simply two entirely different quantities. Whether they could be related by any clever means or not is left here for future investigation.

It is however not enough to stop at the above paragraph; one has to identify what physical quantities $D_{f}$ and $D_{G}$ respectively correspond to. Although surprising it may seem in the first place, I will argue in the next few paragraphs that for fronts with $\delta \phi \rightarrow 0$ at $\xi \rightarrow \pm \infty$ and finite $\tau_{m}$ 's, $D_{f}$ is the long-time front diffusion coefficient, while $D_{G}$ is the short-time (or the instantaneous) diffusion coefficient measured through the Goldstone mode of the front.

The most important point to note is that from its very definition, $D_{G}$ can be measured by tracking the spread in the position of the Goldstone modes for an ensemble of front realizations over an infinitesimally short interval of time; one does not at all need to follow the front realizations over a long period of time. Through its very definition, therefore, $D_{G}$ completely ignores the timecorrelations that exist in the speed fluctuations for individual front realizations of the ensemble (precisely these correlations exhibit themselves through the relaxation time scale $\tau_{m}$ of the $m$-th mode of front shape fluctuation). These correlations do affect the whole dynamics of the individual front realizations, and only $D_{f}$ captures the full flavour of it (c.f. Sec. 4.2), albeit one must pay a price of having to deal with more complicated algebra. Thus, it should not come as a surprise that $D_{f}$ in general yields an expression different from $D_{G}$. The best example that supports this scenario - rather an extreme case where $\tau_{m}$ 's can be arbitrarily large — is the fluctuating "pulled" front (Sec. 4.2.2). Therein, $D_{G}$ simply yield a wrong scaling for the front diffusion as $N \rightarrow \infty$.

In view of existing simulation results on front diffusion, it is worthwhile to revisit the examples for front diffusion in the literature (reviewed in this article) with a critical eye to clarify the above viewpoint.

1. When $R(\phi, \xi, t)$ is replaced by $R\left(\phi^{(0)}, \xi, t\right)$ in Sec. 3.1.2: with the $O\left(\tilde{\varepsilon}^{1 / 2}\right)$ neglected in Eq. (74), in this case, there is no difference between Eqs. (72) and (98). Then, with $D=1$, one obtains

$$
D_{f}=\frac{5 \tilde{\varepsilon}}{2} \int_{-\infty}^{\infty} d \xi g^{2}\left[\phi^{(0)}\right] \neq D_{G}=\tilde{\varepsilon} \frac{\left[\int_{-\infty}^{\infty} d \xi \Phi_{G, L}^{2}(\xi) g^{2}\left[\phi^{(0)}\right]\right]}{\left[\int_{-\infty}^{\infty} d \xi e^{v_{\varepsilon} \xi} \Phi_{G, R}^{2}(\xi)\right]^{2}}
$$

The first point to note for this case is that just like $D_{G}, D_{f}$ also scales as 
$\tilde{\varepsilon}$. The second one is that for pushed fronts, the numerical values of $D_{f}$ and $D_{G}$, in all likelihood, are close by (i.e., one needs good data to distinguish between the two). This is in fact easily seen from the following argument: pushed fronts are strongly localized, and therefore, their Goldstone modes $\Phi_{G, R}$ are localized in space, say around $\xi=\xi_{0}$. Having used this information in Eq. (116), we see that for $D_{G}$, the $e^{v_{\varepsilon} \xi}$ factors in the numerator and the denominator cancel, and thus, effectively apart from some numerical coefficients of $O(1), D_{G} \simeq \tilde{\varepsilon} g^{2}\left[\phi^{(0)}\left(\xi_{0}\right)\right] / \Phi_{G, R}^{2}\left(\xi_{0}\right)$, while $D_{f} \simeq 5 \tilde{\varepsilon} g^{2}\left[\phi^{(0)}\left(\xi_{0}\right)\right] / 2$. How their numerical values compare depends on $\Phi_{G, R}^{2}\left(\xi_{0}\right)$.

To me therefore, the issue regarding the difference between $D_{f}$ and $D_{G}$ for pushed fronts requires further numerical investigation. One particular instance where the above argument breaks down is when the actual front region is very large in size. This is precisely what happens for fluctuating "pulled" (or weakly pushed fronts), i.e., when $\phi^{(0)}$ is the Brunet and Derrida's cutoff solution (11). In that case, there is a clear difference between $D_{f}$ and $D_{G}$ through their respective scalings (also see 3 below).

2. Fluctuating pulled front with multiplicative noise in Sec. 3.3: in this case, it has been clearly shown, numerically and as well as via the Cole-Hopf transformation, that the front wandering is subdiffusive. The first sign of the fact that there is a problem associated with the separation of time scales in this particular example (slow for front wandering and fast for front shape fluctuations) is manifested through the breakdown of convergence of the integrals in the definition of $D_{G}$. A priori, it seems strange that $D_{f}$ [see Eq. (116)] does not suffer from any similar peculiarities.

The reason for the expression of $D_{f}$ not exhibiting peculiarities, however, is not hard to trace. It is in fact hidden within the very derivation of Eq. (116) - recall that there have been cancellations of factors of $\tau_{m}$ all along Sec. 4.2.1. For a pulled front $\tau_{m}$ 's are infinite, and these cancellations are meaningless. The derivation of Eq. (116), thus, simply breaks down for fluctuating pulled fronts with multiplicative noise.

Nevertheless, since $\mathcal{W}(t)$ has been measured operationally via the centre of mass in Ref. [98], it makes sense to use $D_{f}$ to calculate $\mathcal{W}(t)$ as well. With the introduction of the upper cutoff $\xi_{c} \sim \sqrt{4 D t}$, which makes the $\tau_{m}$ 's finite, one can very easily show [having $R(\phi, \xi, t)$ replaced by $R\left(\phi^{(0)}, \xi, t\right)$ ] that

$$
D_{f} \sim \frac{4 \tilde{\varepsilon} D^{5 / 2}}{\sqrt{t}} \neq D_{G} \sim \frac{3 \tilde{\varepsilon}}{v_{\varepsilon}^{2} \sqrt{\pi D t}}
$$

for $t \gg 1$. Interestingly enough, in this example, one gets away with the usage of $D_{G}$ to obtain the theoretical curve (dashed line) for $\mathcal{W}(t)$ in Fig. 32 - both $D_{f}$ and $D_{G}$ scales as $1 / \sqrt{t}$ for $t \gg 1$, and for Fig. 32 that is all that matters.

3. Fluctuating "pulled" fronts, such as the sFKPP equation: although we 
have discussed this at length in Sec. 4.2.2, it is still useful to articulately point out a few distinct subtleties. First, if the noise term in Eq. (96) is made additive by replacing $\phi$ by $\phi^{(0)}$, where $\phi^{(0)}$ is Brunet and Derrida's cutoff solution (11), $D_{f}$ scales as $1 / N$ [c.f. Eq. (116)] for $N \rightarrow \infty$, as opposed to $1 / \ln ^{3} N$. It is therefore important to take notice of this subtlety for simulation purposes - Brunet and Derrida properly take this into account in their own simulations [21]. For $D_{G}$ however, it does not matter whether one uses $\phi$ or $\phi^{(0)}$ for the noise term in Eq. (96) [this is quite obvious from the expressions of $D_{G}$ in Eqs. (107) and (116)], one always gets $D_{G} \sim 1 / \ln ^{6} N$ for $N \rightarrow \infty$.

Secondly, since $D_{G}$ is the short-time (or the instantaneous) diffusion coefficient of the front, if the diffusive spread $\left\langle\Delta x_{G}^{2}(t)\right\rangle$ in the front positions measured through the Goldstone modes are plotted as a function of time for an ensemble of fluctuating "pulled" fronts, one would see that $d\left\langle\Delta x_{G}^{2}(t)\right\rangle / d t=1 / \ln ^{6} N$ right from the very start. On the other hand, when the front positions are tracked by the positions of their centres of mass, one expects $d\left\langle\Delta x_{f}^{2}(t)\right\rangle / d t$ to be smaller for small $t$, but it should nevertheless converge to $1 / \ln ^{3} N$ for $t \rightarrow \infty$. In fact, one can further argue that $d\left\langle\Delta x_{f}^{2}(t)\right\rangle /\left.d t\right|_{t \rightarrow 0} \sim 1 / \ln ^{4} N$. In order to do so, one simply has to observe that the $1 / \ln ^{3} N$ scaling of $D_{f}$ is obtained from $\ln N$ number of modes, each of which contribute an amount of $1 / \ln ^{4} N$ to $D_{f}$ [c.f. Eq. (115)]. The shortest and the longest surviving modes have lifetimes respectively of $O(1)$ and $O\left(\ln ^{2} N\right)$. Thus, $d\left\langle\Delta x_{f}^{2}(t)\right\rangle /\left.d t\right|_{t \rightarrow 0}$ captures only the contribution of the shortest surviving modes and should behave as $\sim 1 / \ln ^{4} N$, while in order to observe the true $1 / \ln ^{3} N$ scaling of $D_{f}$, one has to wait till $O\left(\ln ^{2} N\right)$ time for the contributions of all the $\ln N$ number of modes to $D_{f}$ to add up together.

I finally end Sec. 4 (and this review article too) with the comment that further simulations and research are necessary to confirm the conjectures made in Sec. 4.3 .

\section{Author's Note}

This review article has been written from my own perspective, and it is clearly reflected by the organization of the topics that I have covered. After a brief introduction to the subject, I spent a longish section on the discrete particle and lattice models of fluctuating fronts. Needless to say, there is no unifying underlying structure for the plethora of results in that section - each problem or topic has been dealt with a different approach in the existing literature. The two sections 3 and 4 I spent on field-theory - therein, we find an organized theoretical backbone for studying the effects of fluctuations on 
propagating fronts by means of stochastic differential equations. Finally at the end, I tried to merge the predictions of field-theories on internal fluctuations and the corresponding results for the discrete particle and lattice models.

Unless existing derivations are too involved or too complex, I have tried to outline the main line of procedure. The only exception has been the issues of front diffusion in one spatial dimension, specially for the fluctuating "pulled" fronts, where I have delved deep in the dirty world of algebra. This was done with keeping in mind that front diffusion in one spatial dimension is a relatively new topic. In any case, I have also thrived to put in physical insights wherever appropriate. At various places, open issues have been spelled out for further investigations as well. As for myself, it would be gratifying to see if the expert researchers, and as well as the beginners in this field, both of whom are the target readers, find this review article useful.

\section{Acknowledgement}

A large part of my own work on this subject has been carried out in close collaboration with Wim van Saarloos, whose insight has deeply influenced me. I would also like to thank my one-time colleague (and presently friend) Ramses van Zon for sharing his thoughts on many of the topics discussed here. For many odds and ends of scientific discussions, I am also grateful to (in alphabetical order) Henk van Beijeren, Jaume Casademunt, Jean-Sebastien Caux, Charlie Doering, Wouter Kager, Hans van Leeuwen, Esteban Moro and Bernard Nienhuis. I also wish to thank H.-P. Breuer, Charlie Doering, Wim van Saarloos, Len Sander, Igor Sokolov and Ramses van Zon for giving me prompt permission to use figures from their own papers. Thanks for all the fish!

Financial support has been provided by the Dutch Research Organization FOM (Fundamenteel Onderzoek der Materie).

\section{References}

[1] G. Ahlers and D. S. Cannell, Vortex-front propagation in rotating CouetteTaylor flow, Phys. Rev. Lett. 50, 1583 (1983).

[2] B. Andò and S. Graziani, Stochastic Resonance Theory and Applications, (Kluwer Academic Publishers, Boston, 2000).

[3] J. Armero, J. Casademunt, L. Ramírez-Piscina, and J.M. Sancho, Ballistic and diffusive corrections to front propagation in the presence of multiplicative noise, Phys. Rev. E 58, 5494 (1998). 
[4] J. Armero, J. M. Sancho, J. Casademunt, A. M. Lacasta, L. Ramírez-Piscina, F. Sagués, External Fluctuations in Front Propagation, Phys. Rev. Lett. 76, 3045 (1996).

[5] D. ben-Avraham, Fisher waves in the diffusion-limited coalescence process $A+A \leftrightharpoons A$, Physics Letters A 247, 53 (1998).

[6] D. ben-Avraham, Inhomogeneous steady states of diffusion-limited coalescence $A+A \leftrightarrow A$, Physics Letters A 249, 415 (1998).

[7] D. ben-Avraham, Diffusion-limited coalescence, $A+A \leftrightarrow A$, with a trap, Phys. Rev. E 58, 4351 (1998).

[8] D. ben-Avraham, Complete exact solution of diffusion-limited coalescence $A+A \rightarrow A$, Phys. Rev. Lett. 81, 4756 (1998).

[9] D. ben-Avraham, M. A. Burschka and C. R. Doering, Statics and dynamics of a diffusion-limited reaction: anomalous kinetics, non-equilibrium self-ordering and a dynamic transition, J. Stat. Phys. 60, 695 (1990).

[10] A.-L. Barabasi and H. E. Stanley, Fractal Concepts in Surface Growth, (Cambridge Univ. Press, Cambridge, 1995).

[11] A. Becker and L. Kramer, Linear stability analysis for bifurcations in spatially extended systems with fluctuating control parameter, Phys. Rev. Lett. 73, 955 (1994).

[12] E. Ben-Jacob, H. R. Brand, G. Dee, L. Kramer, and J. S. Langer, Pattern propagation in nonlinear dissipative systems, Physica D 14, 348 (1985).

[13] M. Bramson, Convergence of solutions of the Kolmogorov equation to travelling waves, Mem. Am. Math. Soc. 44, No. 285 (1983).

[14] M. Bramson, P. Calderoni, A. De Masi, P. A. Ferrari, J. L. Lebowitz and R. H. Schonmann, Microscopic selection principle for a diffusion-reaction equation, J. Stat. Phys. 45, 905 (1986).

[15] E. Brener, H. Levine and Y. Tu, Mean-field theory for diffusion-limited aggregation in low dimensions, Phys. Rev. Lett. 66, 1978 (1991).

[16] H. P. Breuer, W. Huber, and F. Petruccione, Fluctuation effects on wave propagation in a reaction-diffusion process, Physica D 73, 259 (1994); Europhys. Lett. 30, 69 (1995).

[17] N. F. Britten, Reaction-Diffusion Equations and Their Applications to Biology (Academic Press, New York).

[18] C. van den Broeck, J. M. R. Parrondo and R. Toral Noise-Induced Nonequilibrium Phase Transition, Phys. Rev. Lett. 73, 3395 (1994).

[19] E. Brunet and B. Derrida, Shift in the velocity of a front due to a cutoff, Phys. Rev. E 56, 2597 (1997). 
[20] E. Brunet and B. Derrida, Microscopic models of travelling wave equations, Comp. Phys. Comm. 122, 376 (1999).

[21] E. Brunet and B. Derrida, Effect of microscopic noise on front propagation, J. Stat. Phys. 103, 269 (2001).

[22] L.-Y. Chen, N. Goldenfeld and Y. Oono, Renormalization group theory and variational calculations for propagating fronts, Phys. Rev. E 49, 4502 (1994).

[23] L.-Y. Chen, N. Goldenfeld, Y. Oono and G. C. Paquette, Selection, stability and renormalization, Physica A 204, 111 (1994).

[24] J. Cook and B. Derrida, Lyapounov exponents of large, sparse random matrices and the problem ofdirected polymers with random complex weights, J. Stat. Phys. 61, 961 (1990).

[25] A. Couairon and J.-M. Chomaz, Absolute and convective instabilities, front velocities and global modes in nonlinear systems, Physica D 108, 236 (1997).

[26] M. C. Cross and P. C. Hohenberg, Pattern formation outside of equilibrium, Rev. Mod. Phys. 65, 851 (1993).

[27] B. Derrida, Mean field theory of directed polymers in a random medium, Phys. Scr. T 38, 6 (1991).

[28] B. Derrida and H. Spohn, Polymers on disordered trees, spin glasses and travelling waves, J. Stat. Phys. 51, 817 (1988).

[29] G. Dee and J. S. Langer, Propagating pattern selection, Phys. Rev. Lett. 50, 383 (1983).

[30] S. J. Di Bartolo and A. T. Dorsey, Velocity selection for propagating fronts in superconductors, Phys. Rev. Lett. 77, 4442 (1996).

[31] C. R. Doering, Microscopic spatial correlations induced by external noise in a reaction-diffusion system, Physica A 188, 386 (1992).

[32] C. R. Doering and D. ben-Avraham, Interparticle distribution functions and rate equations for diffusion-limited reactions, Phys. Rev. A 38, 3035 (1988).

[33] C. R. Doering and D. ben-Avraham, Diffusion-limited coalescence in the presence of particle input:exact results in one dimensions, Phys. Rev. Lett. 62, 2563 (1989).

[34] C. R. Doering, M. A. Burschka and W. Horsthemke, Fluctuations and correlations in a diffusion-reaction system: exact hydrodynamics, J. Stat. Phys. 65, 953 (1991).

[35] C. R. Doering, C. Mueller and P. Smerecka, Interacting particles, the stochastic Fisher-Kolmogorov-Petrovsky-Piscunov equation, and duality, in Proceedings 3rd International Conference Unsolved Problems of Noise (UPoN) and fluctuations in physics, biology and high technology (2002); AIP Conference Proceedings 665, 523 (2003). 
[36] C. R. Doering, C. Mueller and P. Smerecka, Interacting particles, the stochastic Fisher-Kolmogorov-Petrovsky-Piscunov equation, and duality, Physica A 325, 243 (2003).

[37] U. Ebert and W. van Saarloos, Breakdown of the standard perturbation theory and moving boundary approximation, Phys. Rep. 337, 139 (2000).

[38] U. Ebert and W. van Saarloos, Front propagation into unstable states: universal algebraic convergence towards uniformly pulled fronts, Physica D 146, 1 (2000).

[39] U. Ebert, W. van Saarloos and C. Caroli, Propagation and structure of planar streamer fronts, Phys. Rev. E 55, 1530 (1997).

[40] S. Edwards and D. Wilkinson, The surface statistics of a granular aggregate, Proc. R. Soc. London A 381, 17 (1982).

[41] P. C. Fife, Mathematical Aspects of Reacting and Diffusing Systems, Lecture Notes in Biomathematics 28 (Springer, Berlin, 1979).

[42] J. Fineberg and V. Steinberg, Vortex-front propagation in Rayleigh-Bénard convection, Phys. Rev. Lett. 58, 1332 (1987).

[43] R. A. Fisher, The wave of advance of advantageous genes, Ann. Eugenics 7, 355 (1937).

[44] J. García-Ojalvo, A. Hernández-Machado and J. M. Sancho, Effects of external noise on the Swift-Hohenberg equation, Phys. Rev. Lett. 71, 1542 (1993).

[45] G. W. Gardiner, Handbook of Stochastic Methods (Springer, Berlin, 1985).

[46] S. R. de Groot and P. Mazur, Non-equilibrium Thermodynamics, Dover Publications, New York (1984).

[47] T. J. Halpin-Healy and Y. C. Zhang, Kinetic roughening, stochastic growth, directed polymers and all that, Phys. Rep. 254, 215 (1995).

[48] M. van Hecke, C. Storm and W. van Saarloos, Sources, sinks, and wavenumber selection in coupled CGL equations and experimental implications for counterpropagating wave equations, Physica D 134, 1 (1999).

[49] D. Horváth, V. Petrov, S. K. Scott and K. Showalter, Instabilities in propagating reaction-diffusion fronts, J. Chem. Phys. 98, 6332 (1993).

[50] D. Horváth and A. Tóth, Diffusion-driven front instabilities in the chloritetetrathionate reaction, J. Chem. Phys. 108, 1447 (1998).

[51] R. A. L. Jones, L. J. Norton, E. J. Kramer, F. S. Bates and P. Wiltzius, Surfacedirected spinodal decomposition, Phys. Rev. Lett. 66, 1326 (1991).

[52] N. G. van Kampen, Stochastic Processes in Physics and Chemistry, NorthHolland (2003).

[53] M. Kardar, G. Parisi and Y. C. Zhang, Dynamic scaling of growing interfaces, Phys. Rev. Lett. 56, 889 (1986). 
[54] A. R. Kerstein, Computational study of propagating fronts in a lattice-gas model, J. Stat. Phys. 45, 921 (1986).

[55] A. R. Kerstein, A two-particle representation of front propagation in diffusionreaction systems, J. Stat. Phys. 53, 703 (1988).

[56] D. A. Kessler, Z. Ner, and L. M. Sander, Front propagation: precursors, cutoffs, and structural stability, Phys. Rev. E. 58, 107 (1998).

[57] K. Kladko, I. Mitkov and A. R. Bishop, Universal Scaling of Wave Propagation Failure in Arrays of Coupled Nonlinear Cells, Phys. Rev. Lett. 84, 4505 (2000).

[58] A. J. Koch and H. Meinhardt, Biological pattern formation: from basic mechanisms to complex structures, Rev. Mod. Phys. 66, 1481 (1994).

[59] A. Kolmogorov, I. Petrovsky and N. Piscounov, Study of the diffusion equation with growth of the quantity of matter and its application to a biology problem, Bulletin de l'université d'état à Moscou, Ser. int., Section A, 1, 1 (1937).

[60] J. Krug, Origin of scale invariance in growth processes, Adv. Phys. 46, 139 (1997).

[61] J. Krug and H. Spohn, Kinetic roughening of growing surfaces in "Solids Far from Equilibrium", C. Godrèche Ed. (Cambridge Univ. Press, Cambridge 1992).

[62] J. Krug and P. Meakin, Columnar growth in oblique incidence ballistic deposition: Faceting, noise reduction, and mean-field theory, Phys. Rev. A 43, 900 (1991).

[63] J. S. Langer and H. Müller-Krumbhaar, Mode selection in a dendritelike nonlinear system, Phys. Rev. A 27, 499 (1983).

[64] B. P. Lee, Scaling of reaction zones in the $A+B \rightarrow 0$ diffusion-limited reaction, Phys. Rev. E 50, R3287 (1994).

[65] B. P. Lee, Renormalization group calculation for the reaction $k A \rightarrow \emptyset$, J. Phys. A 27, 2633 (1994).

[66] A. Lemarchand, Selection of an attractor in a continuum of stable solutions: Descriptions of a wave front at different scales, J. Stat. Phys. 101, 579 (2000).

[67] A. Lemarchand, A. Lesne, and M. Mareschal, Langevin approach to a chemical wave front: Selection of the propagation velocity in the presence of internal noise, Phys. Rev. E 51, 4457 (1995).

[68] M. A. Karzazi, A. Lemarchand and M. Mareschal, Fluctuation effects on chemical wave fronts, Phys. Rev. E 54, 4888 (1996).

[69] A. Lemarchand and B. Nowakowski, Perturbation of a local equilibrium by a chemical wave front, J. Chem. Phys. 109, 7028 (1998).

[70] A. Lemarchand and B. Nowakowski, Different description levels of chemical wave front propagation speed selection, J. Chem. Phys. 111, 6190 (1999). 
[71] J. Mai, I. M. Sokolov and A. Blumen, Front propagation and local ordering in one-dimensional ireversible autocatalytic reactions, Phys. Rev. Lett. 77, 4462 (1996).

[72] J. Mai, I. M. Sokolov and A. Blumen, Discreteness effects on the front propagation in the $A+B \rightarrow 2 A$ reaction in 3 dimensions, Europhys. Lett. 44, 7 (1998).

[73] J. Mai, I. M. Sokolov and A. Blumen, Front propagation in one-dimensional autocatalytic reactions: the breakdown of classical picture at small particle concentrations, Phys. Rev. E 62, 141 (2000).

[74] J. Mai, I.M. sokolov, V. Kuzovkov and A. Blumen, Front form and velocity in $a$ one-dimensional autocatalytic $A+B \rightarrow 2 A$ reaction, Phys. Rev. E 56, 4130 (1996).

[75] A. Malevanets, R. Careta and R. Capral, Biscale chaos in propagating fronts, Phys. Rev. E 52, 4724 (1995).

[76] N. Manz, V. A. Davydov, V. S. Zykov and S. C. Müller, Excitation fronts in a spatially modulated light sensitive Belousov-Zhabotinsky system, Phys. Rev. E 66, 036207 (2002).

[77] E. Marinari, A. Pagnani and G. Parisi, Critical exponents of the KPZ equation via multi-surface coding numerical simulations J. Phys. A 33, 8181 (2000).

[78] E. Meron, Pattern formation in excitable media, Phys. Rep. 218, 1 (1992).

[79] A. S. Mikhailov, L. Schimansky-Geier and W. Ebeling, Stochastic motion of the propagating front in bistable media, Phys. Lett. A 96, 453 (1983).

[80] E. Moro, Internal Fluctuations Effects on Fisher Waves, Phys. Rev. Lett., 87, 238303 (2001).

[81] E. Moro, Emergence of pulled fronts in fermionic microscopic particle models, Phys. Rev. E 68, 025102 (2003).

[82] J. A. Murray, Mathematical Biology (Springer, Berlin, 1989).

[83] C. Mueller and R. B. Sowers, Random travelling waves for the KPP equation with noise, J. Funct. Anal. 128, 439 (1995).

[84] M. Niklas, M. Lücke and H. Müller-Krumbhaar, Velocity of a propagating Taylor-vortex front, Phys. Rev. A 40, 493 (1989).

[85] E. A. Novikov, Functionals and the random-force method in turbulence theory, JETP 20, 1290 (1965).

[86] D. Panja, Surprising aspects of fluctuating pulled fronts, in Proceedings 3rd International Conference Unsolved Problems of Noise (UPoN) and fluctuations in physics, biology and high technology 2002; AIP Conference Proceedings 661, 539 (2003). 
[87] D. Panja and W. van Saarloos, Fluctuating pulled fronts: The origin and the effects of a finite particle cutoff, Phys. Rev. E 66, 036206 (2002).

[88] D. Panja and W. van Saarloos, Fronts with a growth cutoff but speed higher than the linear spreading speed, Phys. Rev. E 66, 015206(R) (2002).

[89] D. Panja and W. van Saarloos, The Weakly Pushed Nature of "Pulled" Fronts with a Cutoff, Phys. Rev. E 65, 057202 (2002).

[90] D. Panja, G. Tripathy and W. van Saarloos, Front propagation and diffusion in the $A \leftrightarrow A+A$ hard-core reaction on a chain, Phys. Rev. E 67, 046206 (2003).

[91] D. Panja, Asymptotic scaling of the diffusion coefficient of fluctuating "pulled" fronts, to appear in Rapid Comm. Phys. Rev. E; e-print arxiv condmat/0304371.

[92] G. C. Paquette, L.-Y. Chen, N. Goldenfeld and Y. Oono, Structural stability and renormalization group for propagating fronts, Phys. Rev. Lett. 72, 76 (1994).

[93] J. M. Parrondo, C. van den Broeck, J. Buceta and F. J. de la Rubia, Noiseinduced spatial patterns, Physica A 224, 153 (1996).

[94] F. de Pasquale, J. Gorecki and J. Popielawski, On the stochastic correlations in a randomly perturbed chemical front, J. Phys. A 25, 433 (1992).

[95] L. Pechenik and H. Levine, Interfacial velocity corrections due to multiplicative noise, Phys. Rev. E 59, 3893 (1999).

[96] J. Riordan, C. R. Doering, and D. ben-Avraham, Fluctuations and Stability of Fisher Waves, Phys. Rev. Lett. 75, 565 (1995).

[97] A. Rocco, J. Casademunt, U. Ebert, W. van Saarloos, Diffusion coefficient of propagating fronts with multiplicative noise, Phys. Rev. E 65, 012102 (2002).

[98] A. Rocco, U. Ebert and W. van Saarloos, Subdiffusive fluctuations of "pulled" fronts with multiplicative noise, Phys. Rev. E 62, R13 (2000).

[99] A. Rocco, L. Ramírez-Piscina, J. Casademunt, Kinematic reduction of reactiondiffusion fronts with multiplicative noise: derivation of stochastic sharpinterface equations Phys. Rev. E 65, 056116 (2002).

[100] W. van Saarloos, Front propagation into unstable states. II. Linear versus nonlinear marginal stability and rate of convergence, Phys. Rev. A 39, 6367 (1989).

[101] W. van Saarloos, Three basic issues concerning interface dynamics in nonequilibrium pattern formation, Phys. Rep. 301, 9 (1998).

[102] W. van Saarloos, Front propagation into unstable states, submitted to Physics Reports (2003).

[103] W. van Saarloos, M. van Hecke and R. Holyst Front propagation into unstable and metastable states in smectic-C* liquid crystals:linear and nonlinear marginal stability analysis, Phys. Rev. E 52, 1773 (1995). 
[104] E. K. H. Salje, On the kinetics of partially conserved order parameters: a possible mechanism for pattern formation, J. Phys.: Condensed Matter 5, 4775 (1992).

[105] M.-A. Santos and J. M. Sancho, Noise-induced fronts, Phys. Rev. E 59, 98 (1999).

[106] M.-A. Santos and J. M. Sancho, Front dynamics in the presence of spatiotemporal structured noise, Phys. Rev. E 64, 016129 (2001).

[107] L. Schimansky-Geier, A. S. Mikhailov and W. Ebeling, Effect of fluctuation on plane front propagation in bistable nonequilibrium systems, Ann. der Physik (Leipzig) 40, 277 (1983).

[108] L. Schimansky-Geier and Ch. Zülicke, Kink propagation induced by multiplicative noise, Z. Phys. 82, 157 (1991).

[109] F. Schlögl and R. S. Berry, Small roughness fluctuations in the layer between two phases, Phys. Rev. A 21, 2078 (1980).

[110] F. Schlögl, C. Escher and R. S. Berry Fluctuations in the interface between two phases, Phys. Rev. A 27, 2698 (1983).

[111] I. Sendiña-Nadal, A. P. Muñuzuri, D. Vives, V. Pérez-Muñuzuri, J, Casademunt, L. Ramírez-Piscina, J. M. Sancho and F. Sagués, Wave propagation in a medium with disordered excitability, Phys. Rev. Lett. 80, 5437 (1998).

[112] J. M. Starobin and C. F. Starmer, Boundary-layer analysis of waves propagating in an excitable medium: Medium conditions for wave-front-obstacle separation, Phys. Rev. E 54, 430 (1996).

[113] A. Torcini, P. Grassberger and A. Politi, J. Phys. A, Error propagation in extended chaotic systems, 27, 4533 (1995).

[114] G. S. Triantafyllou, K. Kupfer and A. Bers, Absolute instabilities and selfsustained oscillations in the wake of circular cylinders, Phys. Rev. Lett. 59, 1914 (1987).

[115] G. Tripathy, A. Rocco, J. Casademunt and W. van Saarloos, Universality Class of Fluctuating Pulled Fronts, Phys. Rev. Lett. 86, 5215 (2001).

[116] G. Tripathy and W. van Saarloos, Fluctuation and Relaxation Properties of Pulled Fronts: A Scenario for Nonstandard Kardar-Parisi-Zhang Scaling, Phys. Rev. Lett. 85, 3556 (2000).

[117] Y. Tu and M. C. Cross, Chaotic domain structure in rotating convection, Phys. Rev. Lett. 69, 2515 (1992).

[118] C. P. Warren, G. Mikus, E. Somfai and L. M. Sander, Fluctuation effects in an epidemic model, Phys. Rev. E 63, 056103 (2001).

[119] C. P. Warren, E. Somfai and L. M. Sander, Velocity of front propagation in 1-dimensional autocatalytic reactions, Braz. J. Phys. 30, 157 (2000). 
[120] Y. Zel'dovich, G. I. Braenblatt, V. B. Librovich and G. M. Makhviladze, The Mathematical Theory of Combustion and Explosions (Consultants Bureau, New York, 1985).

[121] G. Zocchi, P. Tabeling and M. Ben Amar, Saffman-Taylor plumes, Phys. Rev. Lett. 69, 601 (1992).

[122] R. van Zon, Ph.D. thesis, Univ. of Utrecht, the Netherlands (2000).

[123] R. van Zon and H. van Beijeren, Front propagation techniques to calculate the largest Lyapunov exponent of dilute hard disk gases, J. Stat. Phys. 109, 641 (2002).

[124] R. van Zon, H. van Beijeren and Ch. Dellago, Largest Lyapunov Exponent for Many Particle Systems at Low Densities, Phys. Rev. Lett. 80, 2035 (1998).

[125] R. van Zon, H. van Beijeren and J. R. Dorfman, Kinetic theory of dynamical systems, in Proceedings NATO ASI on "Dynamics: Models and Kinetic Methods for Non-equilibrium Many-Body Systems", NATO Science Series E, Vol. 317; J. Karkheck Ed., 131 (Kluwer, 2000).

[126] R. van Zon, H. van Beijeren, and J. R. Dorfman, Kinetic theory estimates for the Kolmogorov-Sinai entropy and the largest Lyapunov exponents for dilute, hard-ball gases and for dilute, random Lorentz gases, in "Hard Ball Systems" (editor D. Szasz), Mathematical Encyclopaedia of Mathematical Sciences, Vol. 101 (Springer 2000). 